$10 / 34-978 \delta(1)$
$3-7$ SANDIA REPORT

SAND97-8222 • UC-401

Unlimited Release

Printed February 1997

\section{SAND- -97-8222}

$M 9052,92$

\title{
LEAD IODIDE X-RAY AND GAMMA-RAY SPECTROMETERS FOR ROOM AND HIGH TEMPERATURE OPERATION
}

H. HERMON, R. B. JAMES, T. E. SCHLESINGER, E. CROSS, A. ANTOLAK, J. TONEY, B. A. BRUNETT, A. BURGER, Y-C CHANG, K-T CHEN, M. GOORSKY, HOJUN YOON, J. LUND, D. L. MEDLIN, D. H. MORSE, L. SALARY, M. SCHIEBER, K. SHAH, E. SORIA, AND J. VAN SCYOC

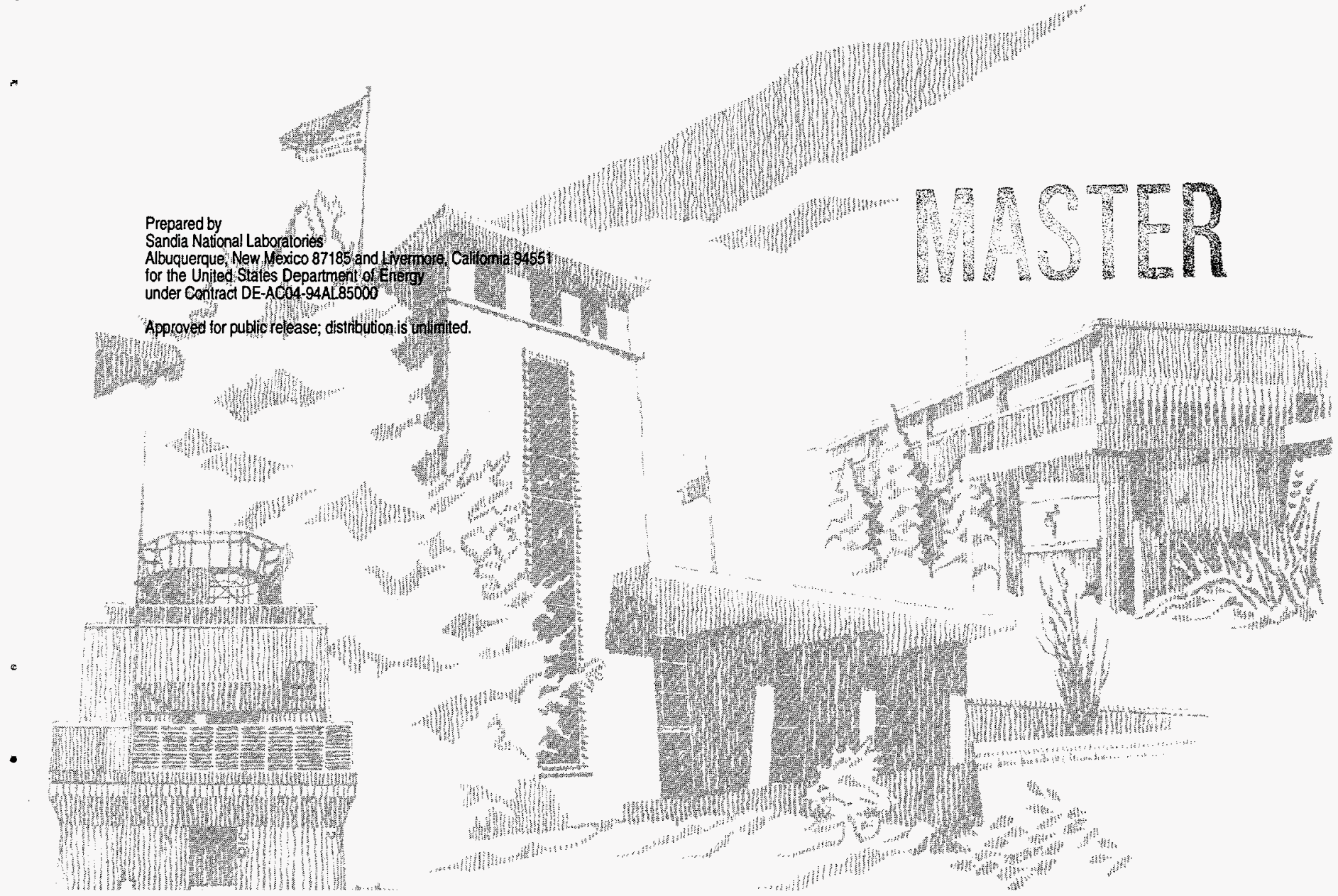


Issued by Sandia National Laboratories, operated for the United States Department of Energy by Sandia Corporation.

NOTICE: This report was prepared as an account of work sponsored by an agency of the United States Govemment. Neither the United States Government nor any agency thereof, nor any of their employees, nor any of the contractors, subcontractors, or their employees, makes any warranty, express or implied, or assumes any legal liability or responsibility for the accuracy, completeness, or usefulness of any information, apparatus, product, or process disclosed, or represents that its use would not infringe privately owned rights. Reference herein to any specific commercial product, process, or senvice by trade name, trademark, manufacturer, or otherwise, does not necessarily constitute or imply its endorsement, recommendation, or favoring by the United States Government, any agency thereof or any of their contractors or subcontractors. The views and opinions expressed herein do not necessarily state or reflect those of the United States Government, any agency thereof, or any of their contractors or subcontractors.

This report has been reproduced from the best available copy.

Available to DOE and DOE contractors from:

Office of Scientific and Technical Information

P.O. Box 62

Oak Ridge TN 37831

Prices available from (615) 576-8401, FTS 626-8401.

Available to the public from:

National Technical Information Service

U.S. Department of Commerce

5285 Port Royal Rd.

Springfield, VA 22161 


\section{DISCLAMMER}

Portions of this document may be illegible in electronic image products. Images are produced from the best available original document. 


\title{
Lead Iodide X-Ray and Gamma-Ray Spectrometers for Room and High Temperature Operation
}

H. Hermon ${ }^{1}$, R. B. James ${ }^{1}$, T. E. Schlesinger ${ }^{2}$, E. Cross ${ }^{1}$, A. Antolak 1 , J. Toney ${ }^{2}$, B. A. Brunett ${ }^{2}$, A. Burger ${ }^{4}$ Y-C Chang 6 , K-T Chen ${ }^{4}$, M. Goorsky ${ }^{3}$, H. Yoon ${ }^{3}$, J. Lund ${ }^{1}$, D. L. Medlin 1 , D. H. Morse ${ }^{1}$, L. Salary ${ }^{4}$, M. Schieber ${ }^{1}$, K. Shah ${ }^{5}$, E. Soria ${ }^{1}$, and J. Van Scyoc ${ }^{3}$

'Sandia National Laboratories, Livermore, CA 94551

${ }^{2}$ Carnegie Mellon University, Pittsburgh, PA 15213

${ }^{3}$ University of California at Los Angeles, Los Angeles, CA 90024

${ }^{4}$ Fisk University, Nashville, TN 37208

${ }^{5}$ RMD Inc., Watertown, MA

${ }^{6}$ University of Illinois at Urbana-Champaign, Urbana, IL 61801

\begin{abstract}
In this study, we report on the results of the investigation of lead iodide material properties. The effectiveness of zone refining purification methods on the material purity is determined by ICP-MS and ICP-OES and correlated to the electrical and physical material properties. We show that this zone refining method is very efficient in removing impurities from lead iodide and we also determine the segregation coefficient for some of these impurities. Triple axis x-ray diffraction (TAD) analysis has been used to determine the crystalline perfection of the lead iodide after applying various cutting, etching, and fabrication methods. The soft lead iodide crystal was found to be damaged when cleaved by a razor blade, but by using a diamond wheel saw, followed by etching, the crystallinity of the material was much improved, as observed by TAD. Low temperature photoluminescence also indicates an improvement in the material properties of the purified lead iodide. Electrical properties of lead iodide such as carrier mobility, were calculated based
\end{abstract}


on carrier-phonon scattering. The results for the electrical properties were in good agreement with the experimental data. 


\section{ACKNOWLEDGMENT}

We gratefully acknowledge the support of the U. S. Department of Energy under this contact. In addition, we would like to acknowledge the efforts of the various graduate students and researchers who have contributed to this effort and have helped to make this project a success. 


\section{PREFACE}

In recent years, the technology of x-ray and gamma-ray detectors that operate at room temperature has improved greatly. Room temperature operated detectors used for x-ray radiation monitoring are very useful for many applications, e.g., in situ analysis of samples, devices used for outer space applications, medical imaging devices, radiation monitoring, preventing nuclear material smuggling, mines detection and basic scientific instrumentation. All systems require a compact, room temperature operated detector. Mercuric iodide $\left(\mathrm{HgI}_{2}\right)$, lead iodide $\left(\mathrm{PbI}_{2}\right)$, and $\mathrm{CdZnTe}(\mathrm{CZT})$ are the leading candidates because of their high atomic number and large band gap that make them particularly well suited for fabrication of high resolution and high efficiency compact room temperature spectrometers.

A great deal of improvement has been made recently on the material purification, crystal perfection and in better understanding the electrical properties of the materials in order to improve detector performance. One of our goals is to find a detector material which can withstand the hostile environment found in nuclear waste storage tanks and facilities (which are too hot for mercuric iodide). Growing single cyrstals of lead iodide is simpler in comparison to mercuric iodide or CZT growth. The difficulty concerning mercuric iodide growth is mainly due to its destructive phase transformation at $127^{\circ} \mathrm{C}$ limiting the growth temperature below the phase transformation point, so the most likely method used is vapor phase growth. The candidate for room temperature operation is CZT which is grown by a unique high pressure Bridgman method, and its growth parameters are now under intensive investigation. On the other hand, the growth conditions of lead iodide are simpler in comparison to the other two. $\mathrm{PbI}_{2}$ material is grown by the well known Bridgman method at relatively low temperature $\left(\sim 400^{\circ} \mathrm{C}\right)$, and there is no need for the complicated growth methods associated with vapor growth for $\mathrm{HgI}_{2}$. High purity starting material $6 \mathrm{~N}$ (99.9999\% purity) is commercially available and further purification, which is crucially important for detector grade lead iodide, is accomplished by zone refining for 100 passes. However, one of the 
major obstacles dealing with lead iodide is its poor mechanical behavior resulting from its layered structure which is very similar to that of mercuric iodide. This structure makes the material very sensitive to mechanical defects such as plastic deformation which degrades electrical properties. As a result, cutting and cleaving of lead iodide crystals would deteriorate its electrical performance which in turn limits the practical detector size in order to achieve higher energy resolution for $\mathrm{x}$ ray spectroscopy. 


\section{SUMMARY}

The objective of this project was to improve the performance and the fabrication process of lead iodide. This work has been done at Sandia and some other locations (e.g., RMD, CMU, UCLA, and LLNL). The role of the laboratory personnel and facilities was to coordinate all the groups and correlate the material and electrical properties of the lead iodide detectors. For this work, we used a variety of analytical techniques including chemical (ICP-MS and ICP-OES), optical (Raman, infrared and photoluminescence), physical (PIXE, XRD, and TAD) properties and $\mathrm{x}$-ray spectroscopy.

Using comprehensive chemical analysis methods (e.g., ICP-OES and PIXE), it was clear that the zone refining method is crucially important in order to reduce chemical impurity levels down to ppm range, which is a basic requirement for detector grade lead iodide. Chemical analysis of the segmented ingot, which are representative of the entire zone refined ingot, enabled us to calculate the segregation coefficient of some major impurities in the lead iodide material. The benefit of the ZR method toward improving material quality was also shown by the low temperature photoluminescence method. To prepare the detector for fabrication, we used the mechanochemical processes which are common to mercuric iodide and lead iodide. This process is more difficult to use for lead iodide because the process involves cutting with a string etchant saw or razor blade. The cutting method successfully used was a diamond wheel saw which, after etching the damaged surfaces, showed significant removal of damage. The chemical etching process was studied, including etch rates characteristic for various etchants solutions (e.g., $\mathrm{KI}, \mathrm{NaI}$, and $\mathrm{Na}_{2} \mathrm{~S}_{2} \mathrm{O}_{3}$ ). Among the three of them, $\mathrm{NaI}$ appears to be the most controlled etch and gave better results. Detectors fabricated from the ZR material gave high energy resolution for low-x-ray energy, but one should note that, at the present time, the performance is limited to small detectors only. 


\section{TABLE OF CONTENTS}

$\begin{array}{ll}\text { Introduction } & 10\end{array}$

$\begin{array}{ll}\text { Background } & 11\end{array}$

Purification of Lead Iodide $\quad 11$

Impurity Analysis $\quad 12$

ICP-OES 12

PIXE Results $\quad 14$

Photoluminescence $\quad 15$

$\begin{array}{ll}X \text { X-Ray Diffraction } & 16\end{array}$

$\begin{array}{ll}\text { Cutting and Chemical Etching } & 17\end{array}$

Phonon Dispersion Characteristic in $2 \mathrm{H}^{\mathrm{PbI}_{2}} \quad 20$

Detector Fabrication and Testing 22

Conclusions $\quad 22$

References $\quad 23$

Figures $\quad 25$

$\begin{array}{ll}\text { Appendix } & 46\end{array}$ 


\section{Lead Iodide X-Ray and Gamma-Ray Spectrometers for Room and High Temperature Operation}

\section{Introduction}

Lead iodide $\left(\mathrm{PbI}_{2}\right)$ is a layered semiconductor material and has a hexagonal structure [1] belonging to the point group P3m1. It was first introduced by Roth and Wilig in 1971 [2-3] and later by others [4] as a n electronic device. The electronic structure and optical properties were studied both experimentally [5] and theoretically [6-8]. $\mathrm{PbI} 2$ is a direct band gap material with a wide band gap (Eg=2.58 eV), high resistivity $\left(10^{13} \Omega \mathrm{cm}\right)$, and because its high atomic number (82, 53), it is well suited for use as a x-ray and gamma-ray radiation detector [9-15]. Its properties revealed much similarity to mercuric iodide. Its advantages over mercuric iodide are lower vapor pressure of the material, higher thermal stability with a melting point of $408^{\circ} \mathrm{C}$ (compare to the destructive phase transformation of $127^{\circ} \mathrm{C}$ of mercuric iodide), a wider energy bandgap and hence lower leakage currents, lower polarization $[13,16]$ and a higher atomic number which provides better stopping power for nuclear radiation. Its disadvantages compared to mercuric iodide are its low mobility $(\mu)$ of $8 \mathrm{~cm}^{2} / \mathrm{Ns}$ and $2 \mathrm{~cm}^{2} / \mathrm{Vs}$ for electrons and holes respectively and lower energy resolution for x-ray radiation [9-11] compared to mercuric iodide, which has electron and hole mobilities of $100 \mathrm{~cm}^{2} / \mathrm{Vs}$ and 4 $\mathrm{cm}^{2} / \mathrm{vs}$. Therefore, in order to enhance the electrical properties, one should improve the trapping time $(\tau)$ characteristics and the mobilities $(\mu)$. Recently, some theoretical calculations (given in this report) shows that the mobility $(\mu$ ) could be much improved on perfect lead iodide crystals. The role of impurities on the electrical properties of lead iodide is crucial, and a great improvement to chemical purification is achieved by using the zone refining method. It is suggested that lead iodide is dominated by structural defects which by eliminating them, its electrical properties should be much improved revealing its actual potential. Some authors suggested that the evidence of polytype formation during 
growth and polytypic phase transformations also influence the electrical behavior. It was found that the polytype structure [17-19], which is mostly $2 \mathrm{H}$ for melt grown lead iodide, is affected by the annealing temperature, etching solvents, purity of the material, and growth conditions. Another known structural imperfection is its fragility caused during growth and during the fabrication process. Because the material is so fragile, even small stresses, pressure or other forces applied to the crystal will result in delamination of the weakly bond layers. Other disadvantages of $\mathrm{PbI}_{2}$ as nuclear radiation detector are low drift mobility, contact degradation, polarization, and multiple nuclear radiation peaks. In this paper, we report on some chemical aspects on lead iodide, electrical properties and theoretical calculation of electron and hole mobilities.

\section{Background}

$\mathrm{PbI}_{2}$ is a material that could replace mercuric iodide for some applications in which the DOE is interested. Some applications require detector material which is tough and rigid to resist environmental conditions or temperatures [20] where mercuric iodide is not capable of collecting data. Such applications are portable remote monitoring of radioactive waste material in which the temperature can easily rise to $80-100^{\circ} \mathrm{C}$. More potential applications include the research nuclear materials by the Nuclear Emergency Search Team (NEST), the verification of international treaties and safeguards, and the satellite detection of nuclear releases. Commercial applications of these detectors and spectrometers include environmental cleanup, sorting of alloys in industry, medicine, radiation monitoring in health physics, and basic science instrumentation. The major obstacle to the widespread use of lead iodide devices is the low manufacturing yield of the detector grade material and the resulting high cost of instruments based on these detectors.

\section{Purification of Lead Iodide}

The detector grade crystals require the highest purity lead iodide available [21]. For that reason, a zone refining [22] (shown in Figure 1) purification method is 
preferred. This method is well described in the literature, and it is based on the differential solubility of the impurities in the melt and in the solid portions of the material. This process involves loading commercially available $6 \mathrm{~N}$ lead iodide as the starting material. The material is introduced into a small diameter ampoule, compacted by melting or by other methods, evacuated (or filled with argon) and sealed off. The ampoule is mounted into the zone refining furnace and the heaters (most likely two ring heaters $8 \mathrm{~cm}$ apart) moved at a speed of $2 \mathrm{~cm} / \mathrm{hr}$ across the length of the ingot and upon reaching the end, quickly moves back to the starting point to prevent melting of the ingot during reverse motion. The furnace temperature was set to $\sim 500^{\circ} \mathrm{C}$ well beyond the melting point of $408^{\circ} \mathrm{C}$. This process was repeated multiple times (about 100 passes) in order to increase the purification efficiency to semiconductor standards. The efficiency of removing an impurity is dependent on the segregation coefficient: the ratio of the solubility of the impurity in the solid to the solubility in the melt. For segregation coefficient much different than unity, there is a better efficiency for removing the impurity. It is shown that detectors fabricated from the sections containing the purest material resulted in best detector performance.

\section{Impurity Analysis}

\section{ICP-OES}

The zone refined lead iodide was analyzed primarily by the ICP-OES method. The method is quite sensitive to metal impurities and the estimated detection limit for most elements was below $1 \mathrm{ppb}$ in the final solution. The samples were immersed in $8 \mathrm{ml}$ aqua regia in a Teflon digestion cell and left overnight. After dilution with deionized water ( $\sim 50 \mathrm{ml}$ ), the samples were placed in CEM 81-D microwave oven and open digested for 40 minutes at $30 \%$ power. A closed vessel digestion was then performed at $50 \%$ power for 40 minutes. Samples were then cooled and transferred to a $100 \mathrm{ml}$ polypropylene Nalgene by volumetric flask with the balance of the volume made up of $10 \%$ aqua regia. The sample was divided into two flasks, the first was spiked with a $2 \mathrm{ml}$ solution with known composition, and a similar volume of $2 \mathrm{ml}$ of aqua regia added to the other. 
Samples from the bottom, middle, and upper sections of the zone refined ingot were analyzed, and the concentrations of a number of impurities $\mathrm{Ca}, \mathrm{Ma}, \mathrm{Fe}, \mathrm{Zn}$, $\mathrm{Al}, \mathrm{Cu}, \mathrm{Mg}, \mathrm{Ni}, \mathrm{Ag}, \mathrm{Mn}, \mathrm{Cr}$ ) were determined. Impurity concentrations for these elements range below about $10 \mathrm{ppm}$, and there is a strong trend for impurities to segregate to the bottom of the ingot, (last to freeze) as a consequence of the zone refining. It also appears that the total impurity concentration is at a minimum towards the middle of the ingot indicating that for some elements the segregation coefficient is below or above unity, so these impurities segregates to the first to freeze or last to freeze parts of the ingot. Figures 2-6 shows the concentration profiles for impurities existing in lead iodide. In this research, we have analyzed some zone refined materials from two sources: RMD company and Fisk University. The results show that the most common impurities (i.e., $\mathrm{Ag}, \mathrm{Cu}, \mathrm{Al}$, $\mathrm{Zn}$ ), and $\mathrm{Mn}$ which are known from other semiconductor materials to be responsible for trapping centers, were found on both sources. Ag was found in the Fisk material (see Figure 5), but very low level in the RMD material (Figure 4). Both ICP-MS and ICP-OES have been used because the sensitivity for some elements is greater in one method compared to the other as shown in Figure 6. From the relative migration of the impurities to one end of the ingot (or to the other end), one can calculate the effective segregation coefficient of the elements in the lead iodide. $\mathrm{Ag}$ and $\mathrm{Cu}$ concentrations, as a function of the position in the ingot, are presented in Figures 8-9. Removing most of the total impurities from the staring material, indicates that the zone refining method is very effective. Using this data, we have computed the segregation coefficient for a number of elements and these are summarized in Table 1. Simplified model for segregation coefficient assumes an impurity concentration, which follows the form $C(x)=A \exp (B x)$, where $x$ is the distance along the ingot and $A$ and $B$ are constants.

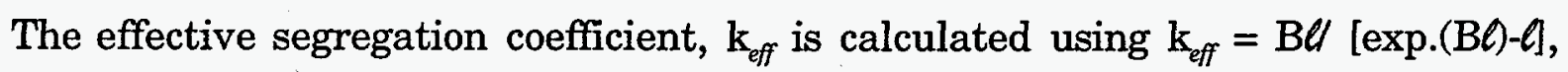
where $\ell$ is the zone length. The constant $A$ is proportional to the initial uniform impurity concentration $C_{0}$ and is expressed as $A=C_{0} B L / \exp (B L-1)$, where $L$ is the ingot length. In view of the good agreement between the model and this data, we expect that these estimates are reasonably accurate. 
TABLE 1.

Elemental Segregation Coefficient $\mathrm{K}_{\mathrm{eff}}$

\begin{tabular}{|l|l|}
\hline Element & $\mathrm{K}_{\text {eff }}$ \\
\hline Chromium & 0.84 \\
\hline Manganese & 0.71 \\
\hline Silver & 0.66 \\
\hline Iron & 0.65 \\
\hline Copper & 0.59 \\
\hline Calcium & 0.55 \\
\hline
\end{tabular}

\section{PIXE Results}

PIXE (Particle Induced X-Ray Emission) was used as a non-destructive technique which enabled us to get a semi-quantitative picture of the impurities at the surface. The PIXE analysis was performed at Lawrence Livermore Laboratories by the Ion Micro-Analysis Group (IMAG). The resulting spectra of $\mathrm{PbI}_{2}$ slices are given in the Table 2 and in Figure 7. Qualitatively, it is clearly shown that the impurity content in the "good" material (yellow-clear) are much lower in comparison to the "bad" (yellow-black) material, even though the results are one order of magnitude higher than those measured by ICP. The most abundant impurities obtain by the PIXE are $\mathrm{Fe}, \mathrm{Cu}, \mathrm{Ni}$, and $\mathrm{Zn}$ which were detected by ICP as well. The high level of $\mathrm{Fe}, \mathrm{Cu}, \mathrm{Ni}$, and $\mathrm{Zn}$ may be due to secondary $\mathrm{x}$-ray fluorescence; nevertheless, zone refining is very efficient at removing impurities proving the efficiency of ZR on removing most of the impurities. 
TABLE 2.

Impurity level (ppm) on ZR crystalline $\mathrm{PbI}_{2}$ as obtain by PIXE

\begin{tabular}{|c|c|c|}
\hline Impurity & Yellowish-Gray Material & Yellow Material \\
\hline Iron & 1100 & 190 \\
\hline Copper & 520 & 430 \\
\hline Nickel & 60 & ND* $^{*}$ \\
\hline Zinc & 150 & ND $^{*}$ \\
\hline
\end{tabular}

*Under detection limit

\section{Photoluminescence}

Low temperature photoluminescence (PL) spectroscopy was performed on lead iodide samples taken from the middle and last to freeze sections of a zone refined ingot. The PL spectra were taken with the samples maintained at $4.2 \mathrm{~K}$ in a low temperature immersion cryostat. The excitation source was an argon ion laser tuned to the $454 \mathrm{~nm}$ line and set at a power level of $40 \mathrm{~mW}$. The laser excitation source was chopped and not focused onto the surface of the sample to avoid excessive excitation power densities. The luminescence was collected from the front surface of the sample and directed to the entrance slits of a SPEX model $14043 / 4 \mathrm{~m}$ spectrometer set to a resolution of about of about 2A. It was then detected by a PMT with an S20 response and associated computer controlled data acquisition system. The photoluminescence spectra as given in Figures 8-11, which are characteristic of the $2 \mathrm{H}$ polytype, may be generally divided into two regions, the near band edge luminescence $(\mathrm{E}=2.52 \mathrm{eV}-2.25 \mathrm{eV})$ and the deeper region $(\mathrm{E}<2.25 \mathrm{eV})$. The spectra are presented with some of the features labeled according to the identification of Brodin et al [23], and a number of observations may be made regarding the observed spectra. The intensity of the near-band-edge luminescence is about a factor of three greater for the spectra obtained from the middle sample of the ingot indicating an overall higher quality material. An increase in this near-band-edge luminescence is probably an indication of a lower concentration of nonradiative or deep recombination centers that could be 
associated with impurities and defects. In the near band edge region of the spectrum, the features are also better resolved again indicating higher quality material. The lower energy luminescence is dominated by two bands centered at about $2.10 \mathrm{eV}$ and $1.72 \mathrm{eV}$ which appear to be somewhat more intense, relative to the near band edge luminescence, in the spectra taken from the last to freeze samples (Figures 8-11) indicating, perhaps, a greater concentration of deeper radiative recombination centers in this material. All these observations are consistent with the trend observed in the ICP-OES studies, namely that the middle of the ingot tends to contain less extrinsic impurities.

\section{X-Ray Diffraction}

The crystals were characterized for their crystallinity by x-ray diffraction spectral analysis (Link analyzer), LXRSA, and by Triple axis X-Ray Diffraction, (TAXRD) and conventional powder diffractometer.

TAXRD measurements were performed with Bede D3 diffractometer. The initial beam $(\mathrm{Cu})$ was conditioned by a (111) $\mathrm{Si}$ channel-cut collimator and a monochromator. The diffracted beam from the sample was further conditioned by a narrow slit, acting as a low resolution analyzer crystal. Rocking curves ( $\omega$ scans), which measure the amount of mosaicity in the crystal, are performed by simply moving the angular position of the sample with the detector fixed in position. X-ray Reciprocal Space Maps (RSM) are performed by taking a series of $\omega-2 \theta$ scans. The RSM measurements allow one to observe both the mosaic structure and the degree of lattice strain variations (due to residual strains, lattice parameter variation, or deviations from stoichiometry). Polycrystalline $\mathrm{PbI}_{2}$ samples (with polytypes $2 \mathrm{H}$ and $4 \mathrm{H}$ ) were analyzed using a conventional $\theta-2 \theta$ coupled diffractometer equipped with a copper x-ray tube. Typical scan range of $5-60^{\circ}$ and a step size of $0.02^{\circ}$ were used for these measurements.

Figures 12-17 shows some x-ray diffraction results that compare samples cut by the diamond wheel saw before and after etching. The mosaic spread is about 250 
and 1000 arcsecs for the best and worst samples, respectively. These results can be compared with thick, cleaved and unetched $\mathrm{PbI}_{2}$ sample (Figure 15), where the mosaic spread is about 250 arcsecs. Figure 16 is a powder scan of $\mathrm{PbI}_{2}$ polytype that shows narrower peaks on the thick sample which is most likely to have less plastic deformations. Figure 17 shows that the diamond saw cut induce more strain and defects to the surface layer but after removing the damaged surface, the bulk is seems to have almost no crystallinity damage. Because of the low $\mu \tau$ product of lead iodide, the thinner detector is preferred rather than the thicker one. Therefore, it is recommended to cut thick slices in order to prevent plastic deformations and to polish it to the desired thickness so all damaged surfaces will be removed from the crystal as described in the next chapter.

\section{Cutting and Chemical Etching}

Chemical etching is used to remove damaged surfaces from the crystal. The thickness of the damaged surfaces is very dependent on the slicing method. Cleaving the crystal perpendicular to the 'c' axis with a razor blade is the most common method used, but it also introduces some mechanical defects to the bulk and roughens the surface. Plastic deformations cannot be easily removed by etching and thicker slices have to be cleaved in order to prevent such defects. The surface of the cleaved slice is usually etched 100-300 microns deep on each side, so all damaged surfaces are removed, then the wafer is ready for the deposition of the contacts. This surface (and near surface region) should be smooth, defect and trap minimized, so that recombination sites for carriers is reduced. In order to reduce the risk of plastic deformation due to cleavage, a string saw is commonly used. The string saw shown in Figure 18 has a cotton thread immersed in $25 \% \mathrm{KI}$. The crystals were mounted on a holder and held in place by wax (B-509). The was heated on a hot plate at $\sim 100^{\circ} \mathrm{C}$ and then applied to the crystal platform. The crystal was placed with orientation of the ' $c$ ' axis facing normal to the travel of the string. One should note that long contact with $\mathrm{KI}$ solution should cause some $\mathrm{KI}$ impurities to migrate into the bulk; therefore, this method is limited to some extent to thick cut slices or bulk cubes which will then be cleaved. Another method which does not involve chemical etching is the use of a diamond wheel saw 
which is described for the first time in this report. Lead iodide crystals were mounted on a graphite substrate as described earlier. In order to reduce heat and mechanical damages, the diamond wheel was set to low speed and it was continuously lubricated with deionized water. About 200-400 $\mu$ each side of the diamond cut slice was removed by chemical polishing. The sample is placed in a flask containing cheese cloth immersed in $10 \% \mathrm{NaI}$, and a random circular motion was employed. The last step after removing the surface damage was the etching process which provides a surface suitable for contact deposition.

Potassium iodide is an effective etchant commonly used for processing $\mathrm{HgI}_{2}$. This solution has been considered for lead iodide and found to introduce more visually observed surface defects (see Figure 19). Thus, we have explored the use of a number of other etching solutions, including sodium iodide (NaI) and sodium thiosulfate $\left(\mathrm{Na}_{2} \mathrm{~S}_{2} \mathrm{O}_{3}\right)$. Etching experiments were performed on a taped cleaved thin lead iodide slice with a known thickness of $\sim 15 \mu \mathrm{m}$. The slice was then divided into small pieces of $5 \mathrm{~mm}^{2}$ in area. All samples tested were of the identical dimensions. We tested $\mathrm{KI}$ and $\mathrm{NaI}$ solutions of various concentrations and also tested $\mathrm{Na}_{2} \mathrm{~S}_{2} \mathrm{O}_{3}$ as well. Etching is accomplished by immersing the samples in the etchant solution which was slightly stirred. Table 3 shows the results of the etching experiments. We found that the $\mathrm{NaI}$ solution is the preferred etchant for $\mathrm{PbI}_{2}$ as determined by visual inspection of the samples (using an optical microscope). No significant differences were observed in terms of etching characteristics between samples etched by $10-30 \%$ solution for short time (2-5 min.) etching. However, at longer times, triangular etch pits became clearly visible on the surface as shown in Figure 19. Some redeposited material was observed as well. Using a stronger $\mathrm{Na}_{2} \mathrm{~S}_{2} \mathrm{O}_{3}$, etchant results in a rapid reaction which caused the sample to flake apart. It appears that further investigation of these solutions at other temperatures or concentrations may yield improved etching characteristics. In order to correlate etching with the electrical properties, further testing has to be done using various etching methods. 
TABLE 3.

Etching Rates for lead iodide (slightly stirred)

\begin{tabular}{|l|l|l|}
\hline \multicolumn{1}{|c|}{ Etching Solution } & Etch Rate & \multicolumn{1}{|c|}{ Comments } \\
\hline $30 \% \mathrm{NaI}$ & $1 \mu / \mathrm{min}$ & Etch pits after longer times \\
\hline $10 \% \mathrm{NaI}$ & $0.5 \mu / \mathrm{min}$ & Optically clear \\
\hline $30 \% \mathrm{KI}$ & $3 \mu / \mathrm{min}$ & $\begin{array}{l}\text { Changed to opaque in } \\
\text { appearance }\end{array}$ \\
\hline $10 \% \mathrm{KI}$ & $1.2 \mu / \mathrm{min}$ & \\
\hline $10 \% \mathrm{Na}_{2} \mathrm{~S}_{2} \mathrm{O}_{3}$ & $\mathrm{NA}$ & $\begin{array}{l}\text { Rapid reaction caused sample } \\
\text { to flake apart }\end{array}$ \\
\hline
\end{tabular}

TAD measurements of diamond sawn lead iodide show the near surface region $(<10 \mu \mathrm{m})$ of the material to be significantly damaged (Figures 12 and 17). The overall reflected $\mathrm{x}$-ray intensity is reduced and the line width is quite broad (Figure 12). However, after polishing out the damaged surface and etching for two to five minutes with gentle agitation in a $10 \%$ by weight $\mathrm{NaI}$ solution, a significant improvement in the triple axis x-ray reciprocal space map is seen (see Figures 1314). The intensities of the reflected peaks arc increased and the linewidth of these features are decreased, both indicating an improved crystallinity in the near surface region. After a longer etch time, which removes all surface damage due to diamond sawing, triple axis x-ray diffraction measurements revealed no plastic deformation induced in the bulk ( $>1 \mathrm{~mm}$ ) in contrast to cleaved or tape peeled slices which do show plastic deformation. Thus, we conclude that chemical etching can be used to controllably remove the damaged near surface region that results from the diamond sawing of lead iodide. These results showed improved crystal near surface quality in comparison to cleaving with a razor blade. The performance of spectrometers fabricated from the improved material has been evaluated and pulse height spectra obtained for both ${ }^{55} \mathrm{Fe}$ and ${ }^{241} \mathrm{Am}$ have been reported elsewhere [9-16]. 


\section{Phonon Dispersion Characteristic in $\mathbf{2 H} \mathbf{P b I}_{2}$}

The carrier mobilities are reported in the literature are obtained by various methods (i.e., Hall measurements [24] and I-V studies). In this chapter, a theoretical calculation of the phonon modes and carrier transport properties are reported using polar optical scattering measurements [25]. Since $\mathrm{PbI}_{2}$ is anisotropic, we expect that the long wavelength optical phonon modes will display angular dispersion. The angular dispersion for $\mathrm{E}_{\mathrm{u}}$ is quite large, and tends to cross the dispersion curve of the $A_{2 u}$ mode. These are the only lines that show angular dispersion. This angular dependence plays a significant role in determining the anisotropy of the carrier mobilities. In the $E_{u}$ and $A_{2 u}$ optical modes, ions with opposite charges vibrate against each other, thus giving rise to a long term macroscopic electric field. The interaction of a carrier with such field is known as the polar optical scattering. Due to the strong polarity of the material, it is expected that polar-optical scattering will be the dominant mechanism which determines the intrinsic carrier mobility at temperature above $77 \mathrm{~K}$. This phenomena has also been found in the similar $\mathrm{HgI}_{2}$ material. The mobilities are related to the momentum relaxation times via:

$$
\mu \ell=\mathrm{e} / \mathrm{m} \ell^{*} \mathrm{x}<\mathrm{E} \tau \ell(\mathrm{E})>/<\mathrm{E}>\quad \mu \tau=\mathrm{e} / \mathrm{m}_{\mathrm{t}}{ }^{*} \mathrm{x}<\mathrm{E} \tau_{\mathrm{t}}(\mathrm{E})>/<\mathrm{E}>
$$

Where $<>$ denotes a thermal average with a Boltzmann distribution, $\mu_{t}$ is longitudinal mobility and $\mu_{\mathrm{t}}$ is transverse mobility, $\mathrm{m}_{\mathrm{i}}$ is the effective mass, and $\tau_{\mathrm{i}}$ is the momentum relaxation time, the subscript ${ }_{i}$ denotes the direction of transport. The detailed calculations and results for electron photon interaction are given in the appendix.

Figure 20 shows the mobility as a function of temperature for carrier with $\mathrm{m}_{\ell}^{*}=$ $\mathrm{m}_{0}$ and with three different values of $\mathrm{m}_{\mathrm{t}}{ }^{*} / \mathrm{m}_{t}^{*}$ which defined as $\sigma=1,2$ and 5 . The effective mass $m_{i}$ and the electron mobility $\mu \mathrm{I}$ were calculated. The following values were obtained (assuming $\sigma=2$ ): $\mathrm{m}_{\imath}^{*} \approx 0.96 \mathrm{~m}_{0}$ (which is greater than $\mathrm{m}_{\mathrm{t}}^{*} \approx$ $\left.0.48 \mathrm{~m}_{0}\right)$, and the electron mobility $\mu_{t}\left(\mu_{\mathrm{t}}\right) \approx 110(140) \mathrm{cm}^{2} / \mathrm{V}_{\mathrm{s}}$ and $20(25) \mathrm{cm}^{2} / \mathrm{v}_{\mathrm{s}}$ at $100 \mathrm{~K}$ and $300 \mathrm{~K}$ respectively. Apparently, the calculated mobilities are three times higher than the experimental results; therefore, we can estimate that the 
intrinsic mechanism due to polar optical scattering is one of the dominant factors in lowering the mobilities. We expect lead iodide can be improved even more by eliminating all extrinsic scattering. 


\section{Detector Fabrication and Testing}

The lead iodide detectors were fabricated by various methods. The common method for fabrication of the lead iodide detectors (from the already etched slices) involves applying Aquadag contacts on both sides of the detector. At the present time, the best energy resolutions are obtained when small area electrodes are applied. Evaporation of gold and palladium contacts on lead iodide has also been used, but no significant improvement in energy resolution was observed. The typical electronic setup consisted of a Tennelec 170D charge sensitive preamplifier, linear amplifier, low voltage power supply, high voltage power supply, and multichannel analyzer. The best energy resolution has been achieved for a $\sim 100$ micron thick and $2 \times 2 \mathrm{~mm}$ detector prepared by the tape peeling method. The energy resolution measured for this detector was $495 \mathrm{eV}$ FWHM for ${ }^{55} \mathrm{FeK} \alpha$ $(5.9 \mathrm{keV})$ peak as shown in Figure 21.

\section{Conclusions}

In conclusion, we have shown that zone refining is effective in reducing the concentrations of many impurities in lead iodide. In addition, we have obtained the segregation coefficients for a number of these impurities. The material quality is improved after zone refining as shown by low temperature PL measurements. The combination of proper chemical etching and an improved cutting and polishing process based on use of a diamond wheel saw were found to reduce the amounts of defects at the surface are found to be promising. With the appropriate processing techniques, it has been found that detectors fabricated from high purity crystals exhibit significant improvement in performance compared to those produced from low purity crystals. However, problems still exists in lead iodide due to low charge carrier collection efficiency and this low efficiency is probably caused by additional impurities or defects incorporated during crystal growth and detector fabrication processes. In addition, we are currently investigating the use of pulse processing techniques to improve energy resolution and detector efficiency. 


\section{References}

1. B. Palosz, Phys. Stat. Sol. (a) 80,11 (1983).

2. S. Roth and W. R. Willig, Appl. Phys. Lett. 18, 328 (1971).

3. W. R. Willig, Nucl. Inst. and Meth., 101 (1972) 23.

4. C. Manfredotti, R. Murri, A. Quirini, and L. Vasanelli, IEEE Trans. Nuc. Sci. NS-24, 126 (1977).

5. G. Baldini and S. Franch, J. Phys. Rev. Lett., 26, 503 (1971).

6. L. Ch. Schluter and M. Schluter, Phys. Rev. B9, 1652 (1954).

7. F. Olschner, J. C. Lund, K. S. Shah, and M. R. Squillante, ICFA Instrum. Bull. 7,9 (1989).

8. R. Minder, G. Ottaviani, and C. Canali, J. Appl. Physc. Chem. Solids 37, 417 (1976).

9. J. C. Lund, K. S. Shah, M. R. Squillante, and F. Sinclair, IEEE Trans. Nuc. Nci.

NS-35, 89 (1988).

10. J. C. Lund, K. S. Shah, M. R. Squillante, L. P. Moy, F. Sinclair, and G. Entine, Nucl. Inst and Meth. A283, 299 (1989).

11. J. C. Lund, K. S. Shah, F. Olschner, J. Zhang, L. P. Moy, F. Medrick, and M. R. Squillante, Nucl. Inst and Meth. A322, 464 (1992).

12. J. Zhang, K S. Shah, F. Olschner, J. C. Lund, L. P. Moy, K Daley, L. Cirignano, and M. R. Squillante, Nucl. Inst and Meth. A322, 499 (1992).

13. D. C. Dominique, R. B. James, H. Feemster, R. Anderson, A. J. Antolak, D. H. Morse, A. E. Pontau, H. Jayatirtha, A. Burger, X. J. Bao, T. E. Schlesinger,

G. S. Bench, and D. W. Heikkinen, Mat. Res. Symp. Proc. 302, 335 (1993).

14. V. Deich and M. Roth, Nucl. Instr. and Meth., (1996) in press.

15. J. C. Lund, F. Olschner and A. Burger, in Semiconductors for Room Temperature Nuclear Detector Applications, edited by T. E. Schlesinger and R. B. James in Semiconductors and Semimetals, 43 (Academic Press, San Diego, 1995), 443.

16. D. C. David, A. Burger, W. Wang, R. B. James, T. E. Schlesinger, and J. C. Lund, Proc. SPIE, 1734 (1992) 146.

17. M. Rao and O. N. Srivastava, J. Phys. D: Appl. Phys. 11, 919 (1978). 
18. S. K. Chaudhary and G. C. Trigunayat, J. Crystal Growth 62, 398 (1983).

19. M. Chand and G. C. Trigunayat, J. Crystal Growth 39, 299 (1977).

20. M. A. George, M. Azoulay, H. N. Jayatirha, Y. Biao, A. Burger, W. E. Collins, and E. Silberman, J. of Crystal Growth 137, 299 (1994).

21. J. Eckstein, B. Erier, and K. W. Benz, Mat. Res. Bull. 27, 357 (1992).

22. W. G. Pfann, "Zone Refining," Robert E. Kreiger Publishing Co., Huntington, New York (1978).

23. M. S. Brodin, A. O. Gushcha, L. V. Taranenko, V. V. Tishchenko, V. N. Khotyaintsev, S. G. Shevel, Sov. Phys. Solid State 28, 1658 (1986).

24. P. D. Bloch, W. J. Hodby, T. E. Jenkins, D. W. Stacey, G. Lang, F. Levi, and C. Schwab, J. Phys. C: Solid State Phys. 11, 4997 (1978).

25. Y. C. Chang and R. B. James, to be published (1997). 
Figure 1. Schematic representation of a computer controlled multipass zone refining furnace.

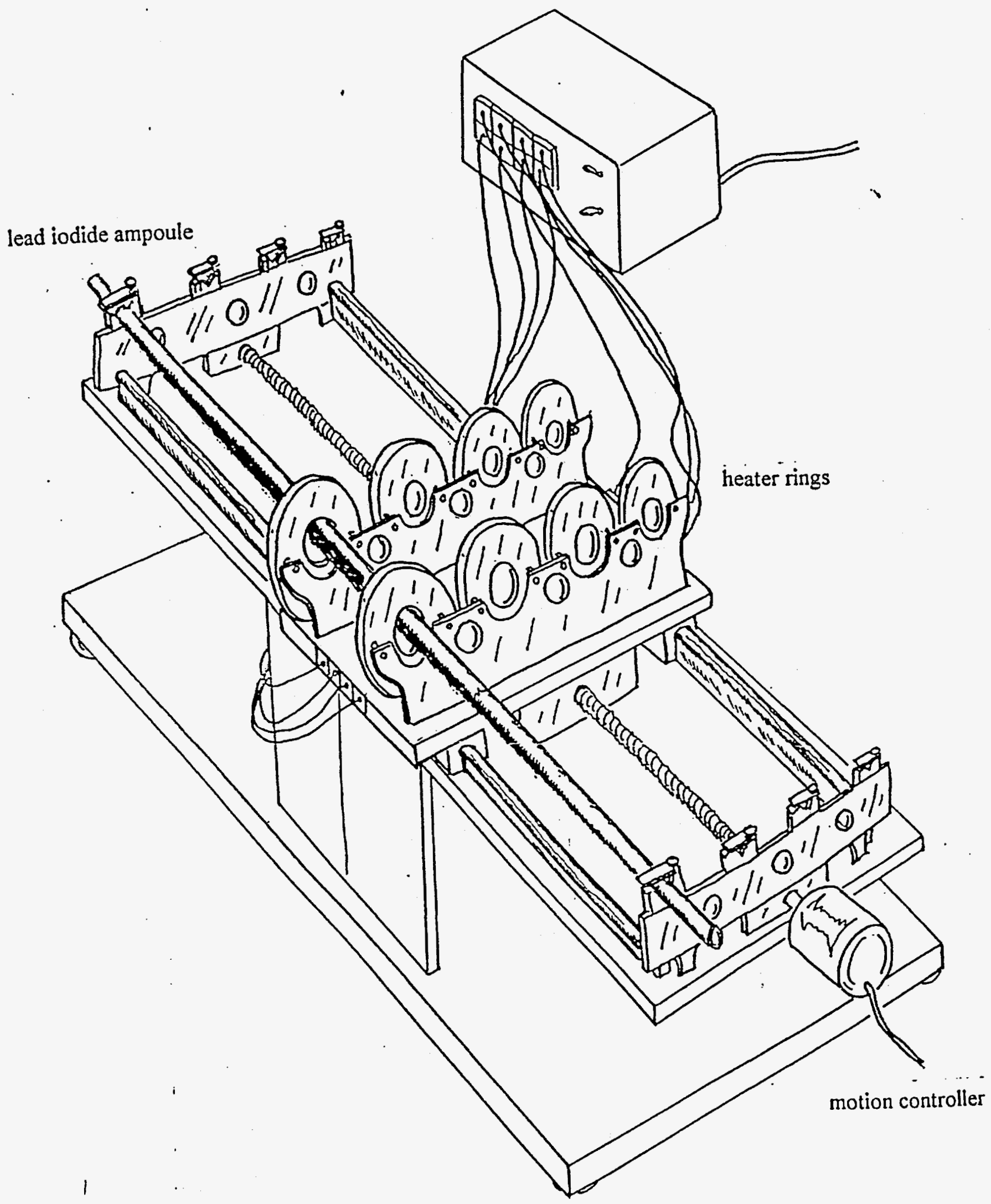


Figure 2. ICPOES elemental analysis of vacuum zone refined lead iodide.

\section{Lead iodide Zone Refined In Vacuum}

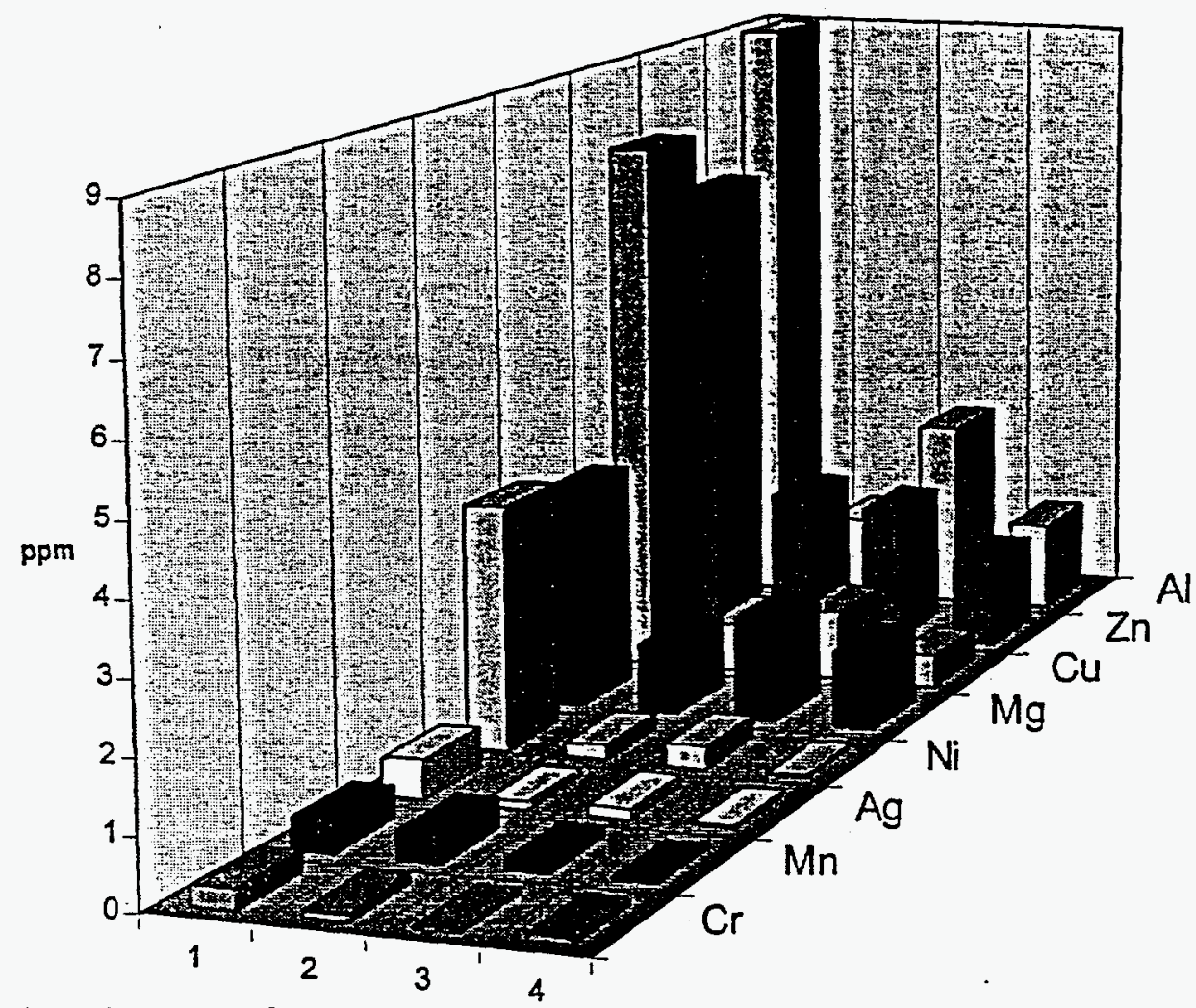

Position Along Zone

Refined Ingot

Heater Travel direction

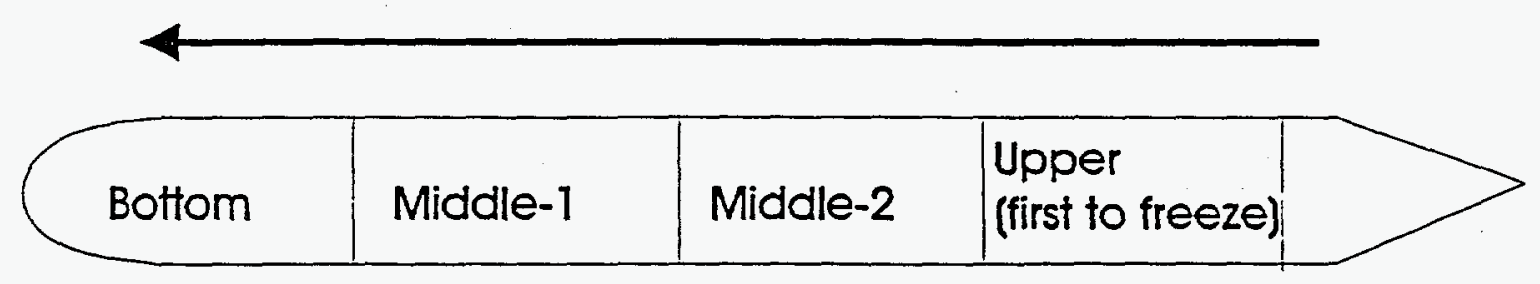


Figure 3. ICPOES elemental analysis of argon backfilled zone refined lead iodide.

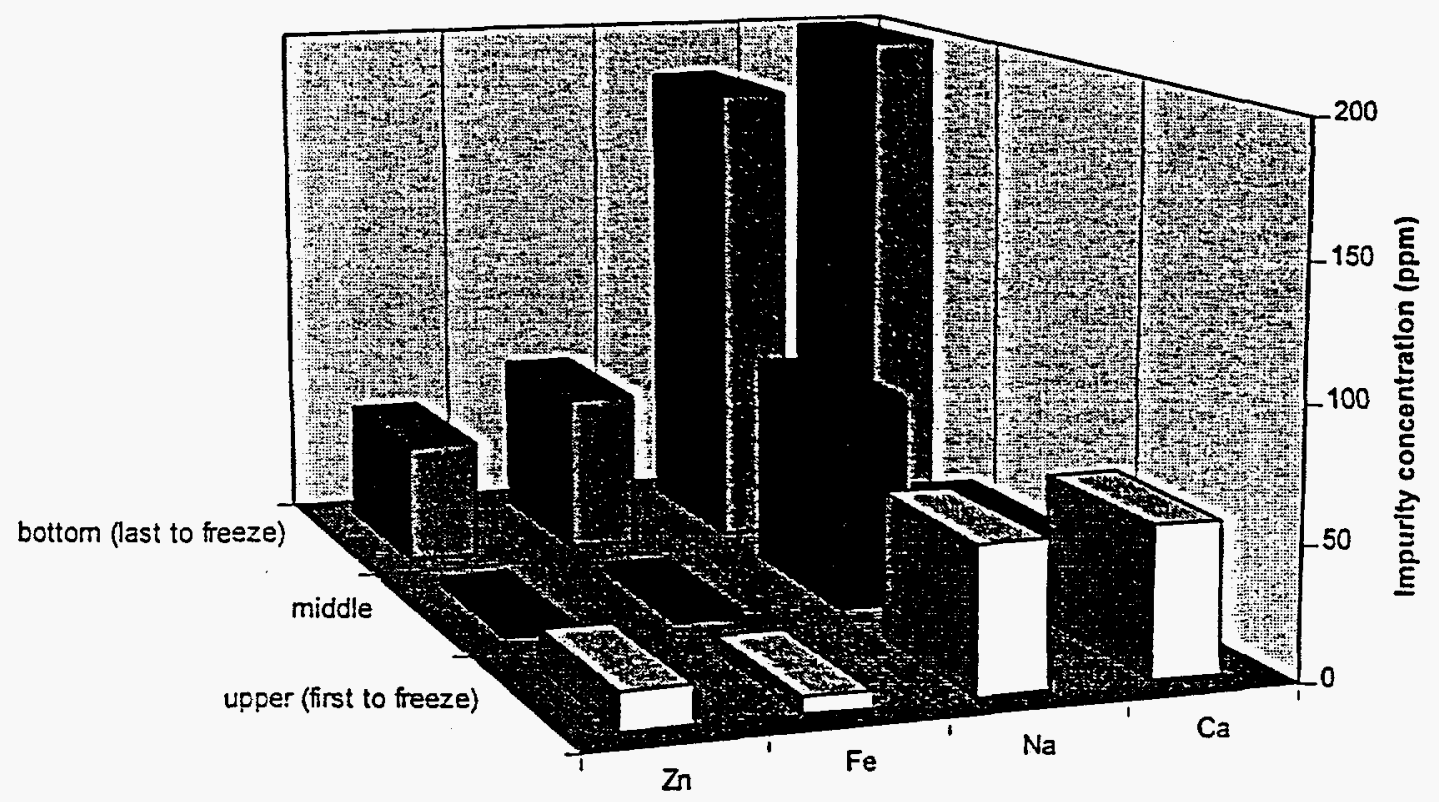


Figure 4. Elemental analysis of zone refined $\mathrm{PbI}_{2}$ ingot (RMD) by two methods: ICP-OES and ICP-MS.
A: First to freeze
B: Last to freeze

Zone refined ingot (RMD) by two methods: ICP-OES and ICP-MS.

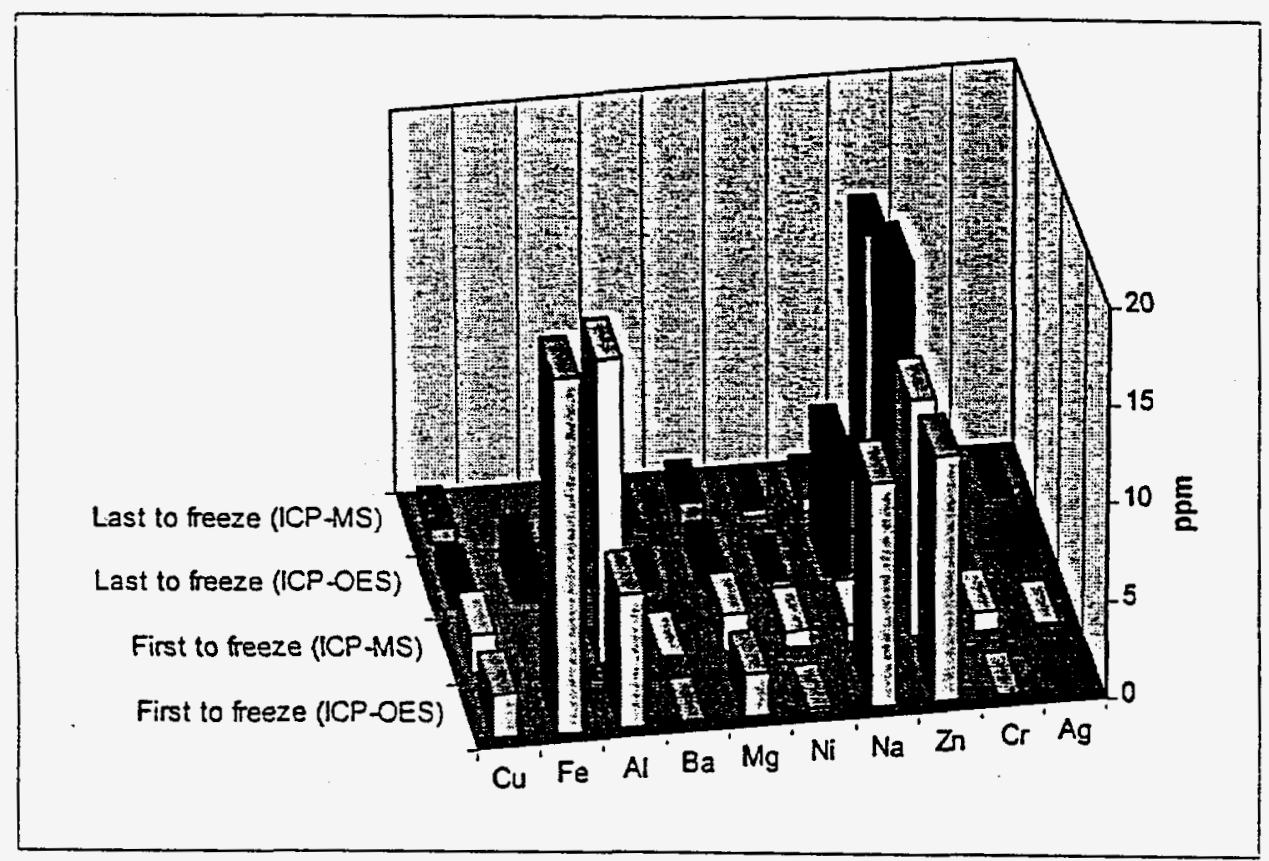


Figure 5. ICPOES elemental analysis of lead iodide from Fish University prior zone refining.

FISK ZR Pbi2

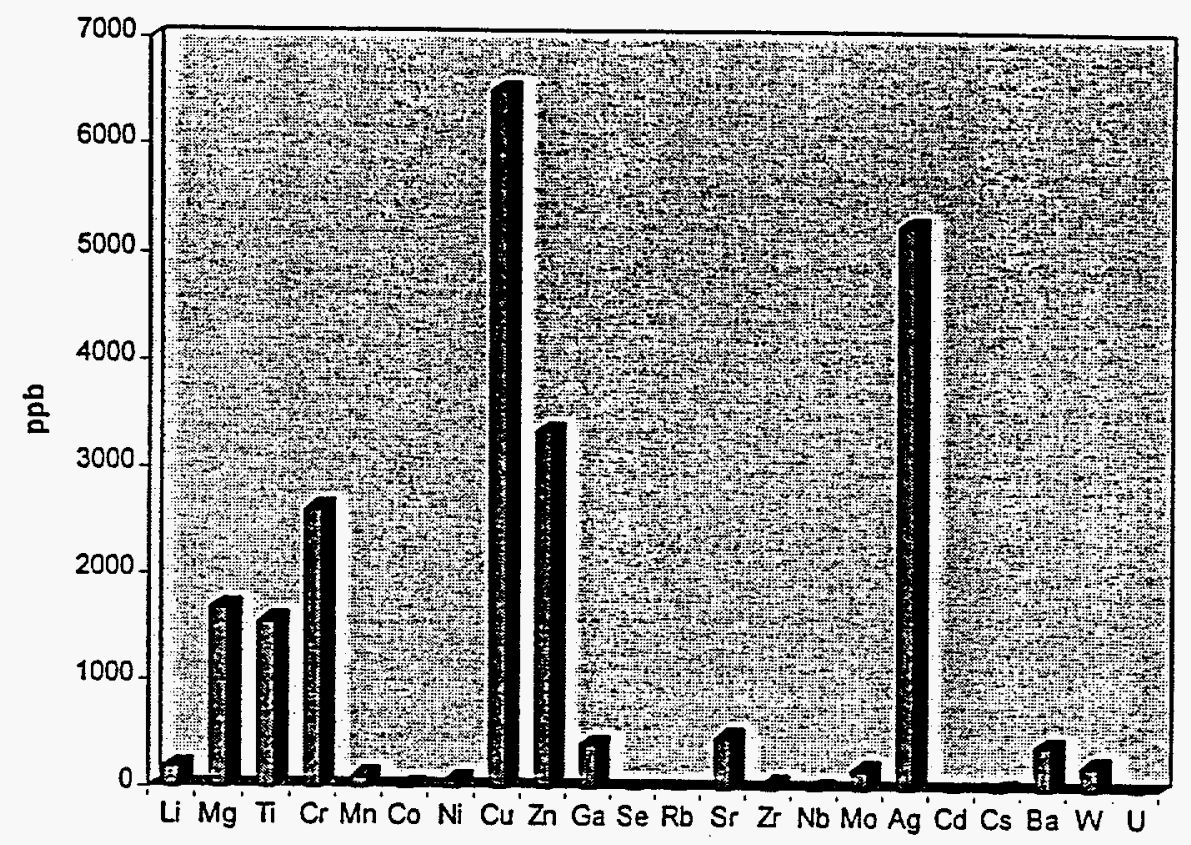


Figure 6. Concentration profile of $\mathrm{Cu}$ and $\mathrm{Ag}$ in the zone refined ingot.

Cu concentration profile in $\mathrm{Pbl}$

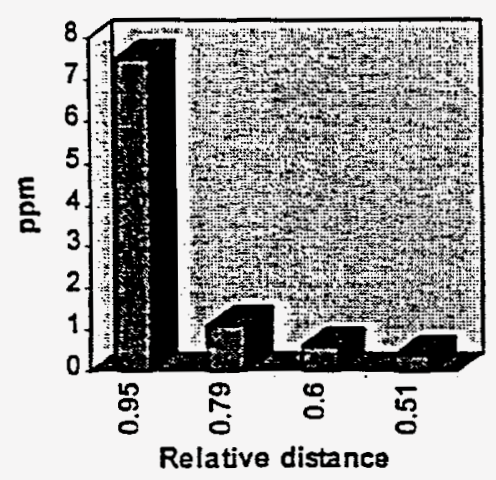

Ag concentration profile in $\mathrm{Pbl2}$

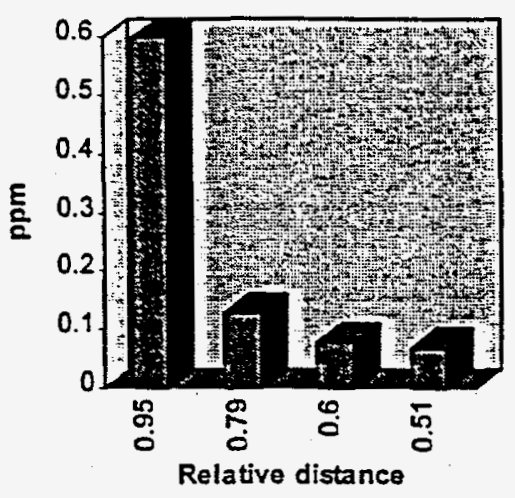


Figure 7. PIXE chemical analysis of zone refined material obtained from the last to freeze (upper spectrum) and from the middle ingot.
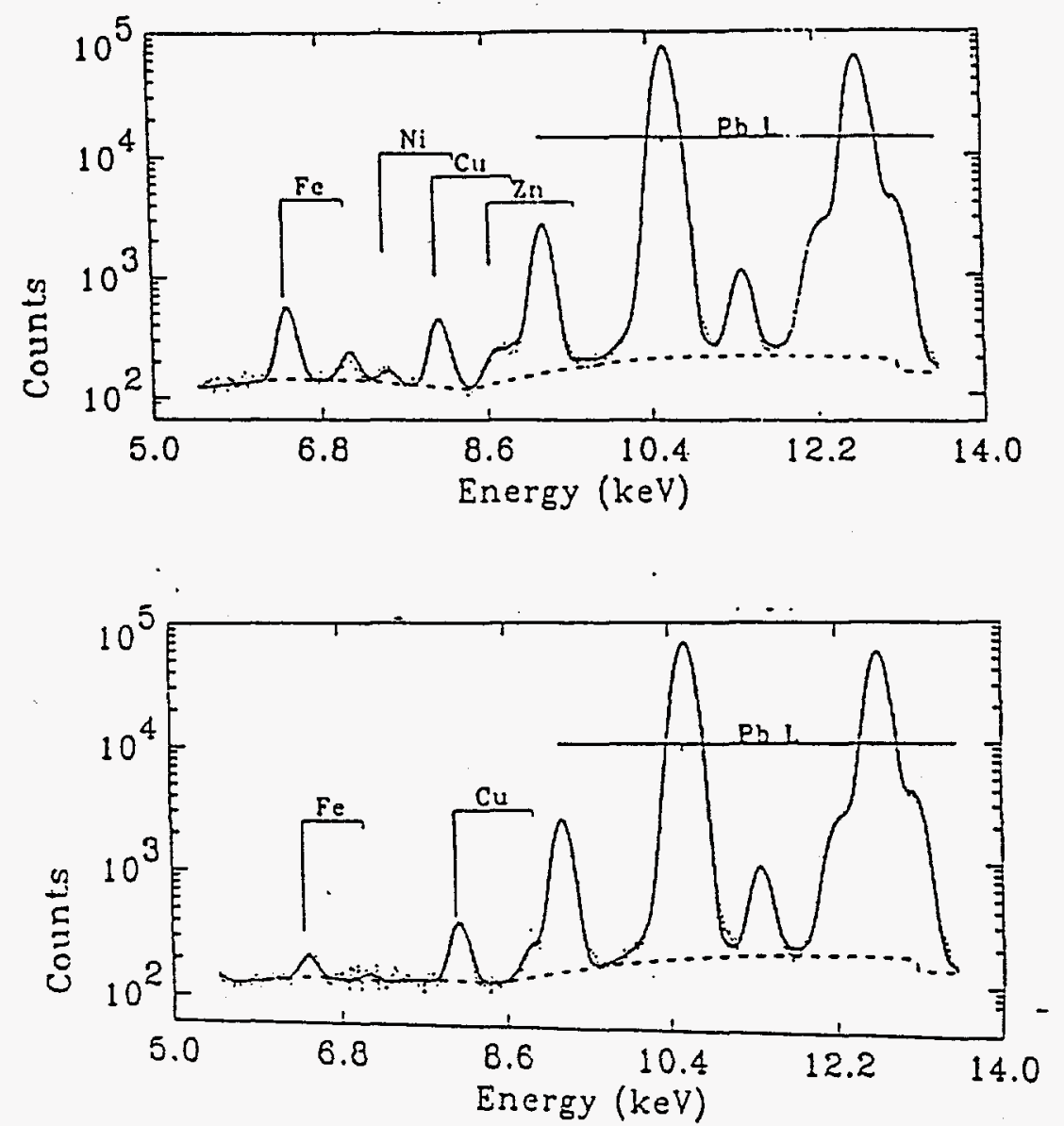
Figure 8. Low temperature $(4.2 \mathrm{~K})$ photoluminescence spectra of zone refined lead iodide samples obtained from the middle (upper spectrum) and the last to freeze (lower) region of an ingot.

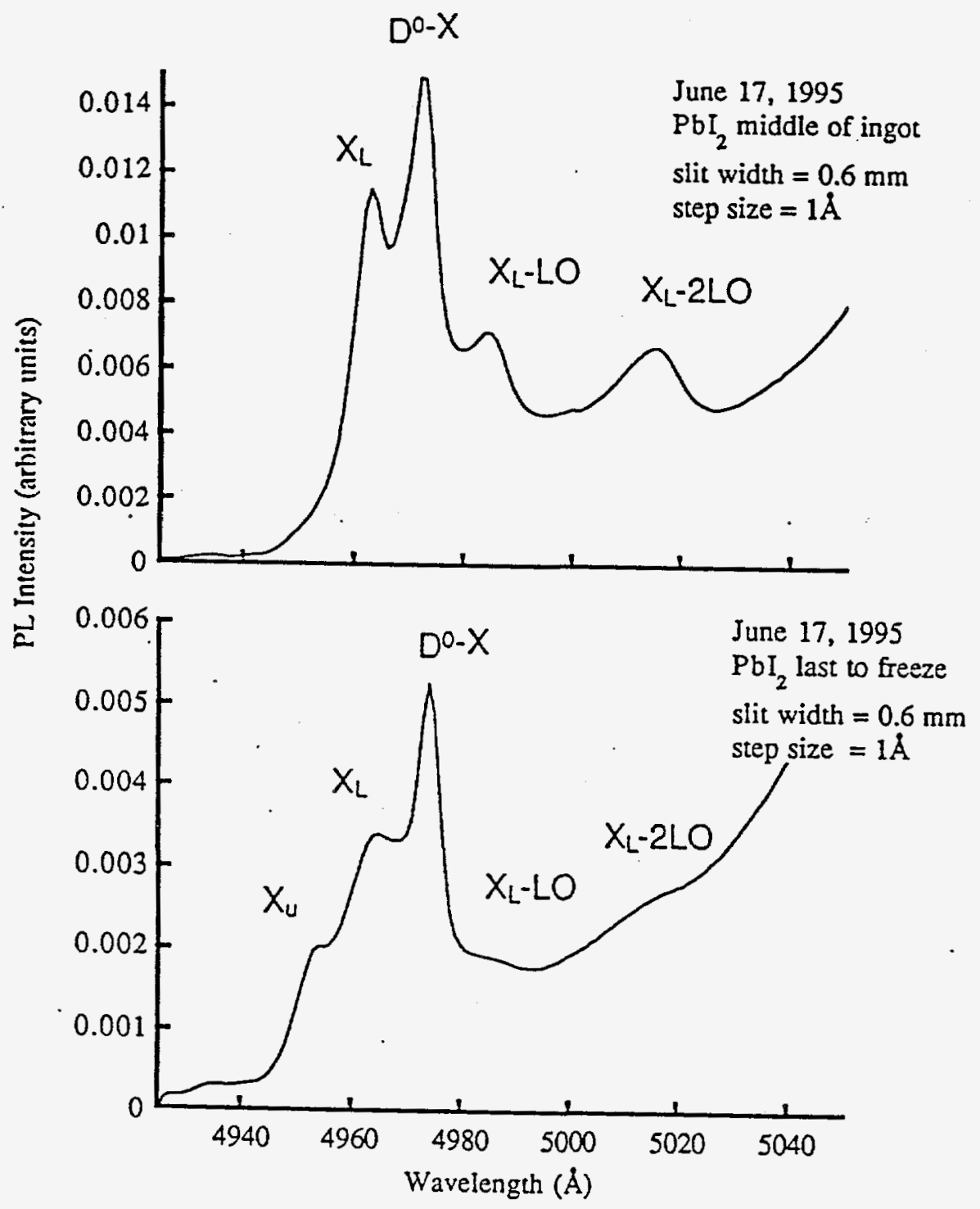


Figure 9. Near band gap photoluminescence of the samples described in Figure 8.

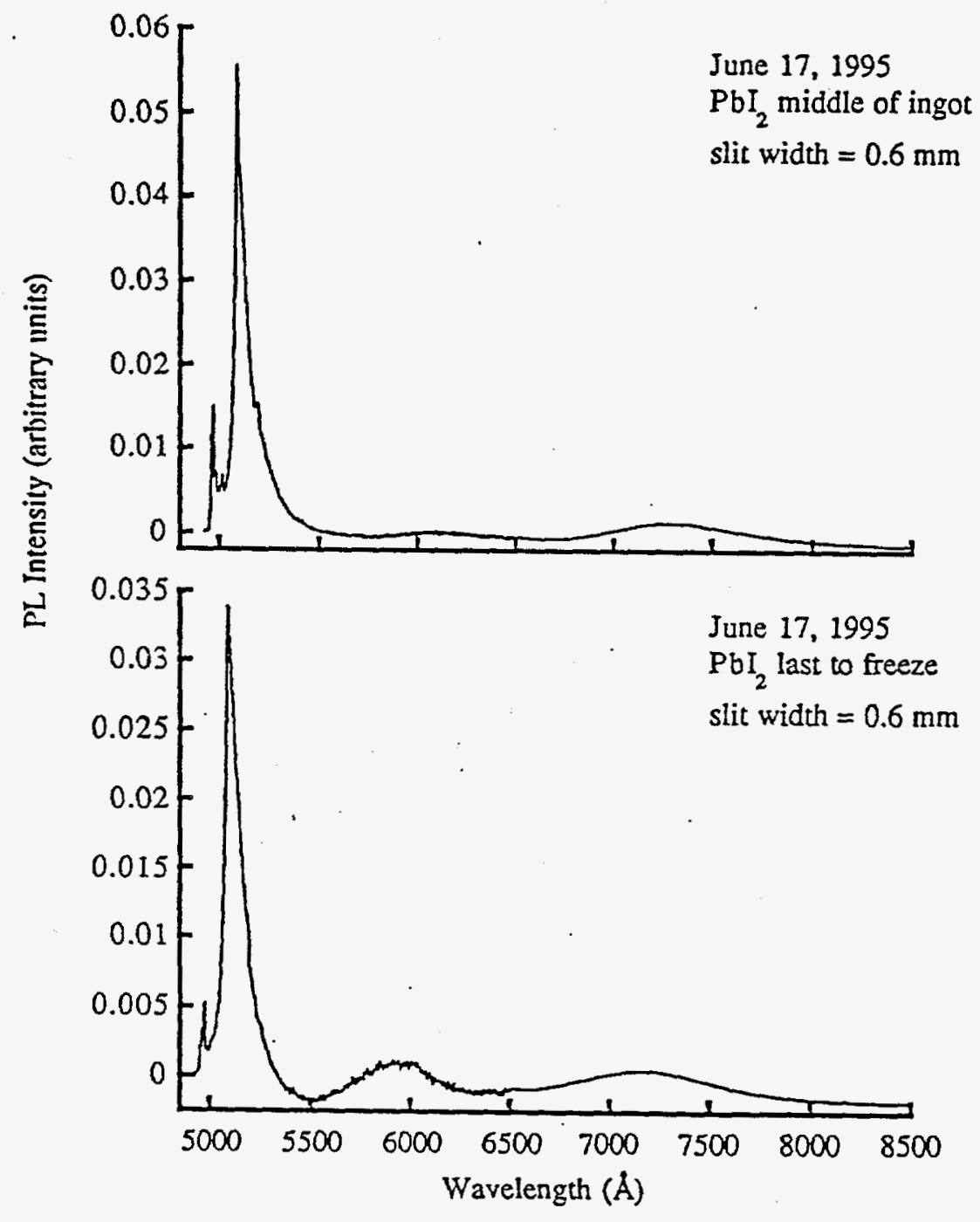


Figure 10. Low temperature $(4.2 \mathrm{~K})$ photoluminescence spectra and near band gap photoluminescence of RMD ingot, middle vs. last to freeze. Note the lower PL intensity from the last to freeze sample.

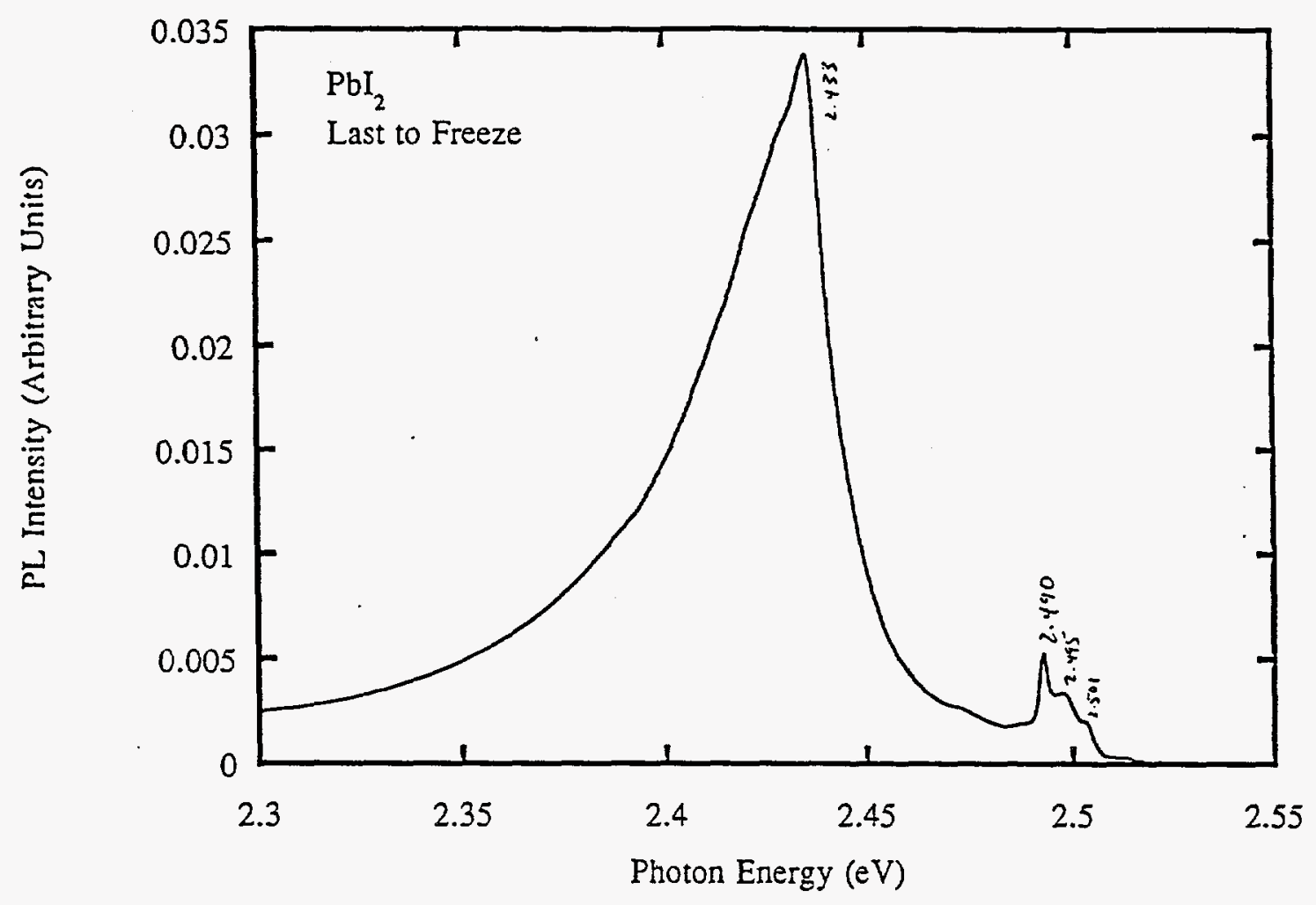


Figure 11. Near band gap photoluminescence of last to freeze ingot.

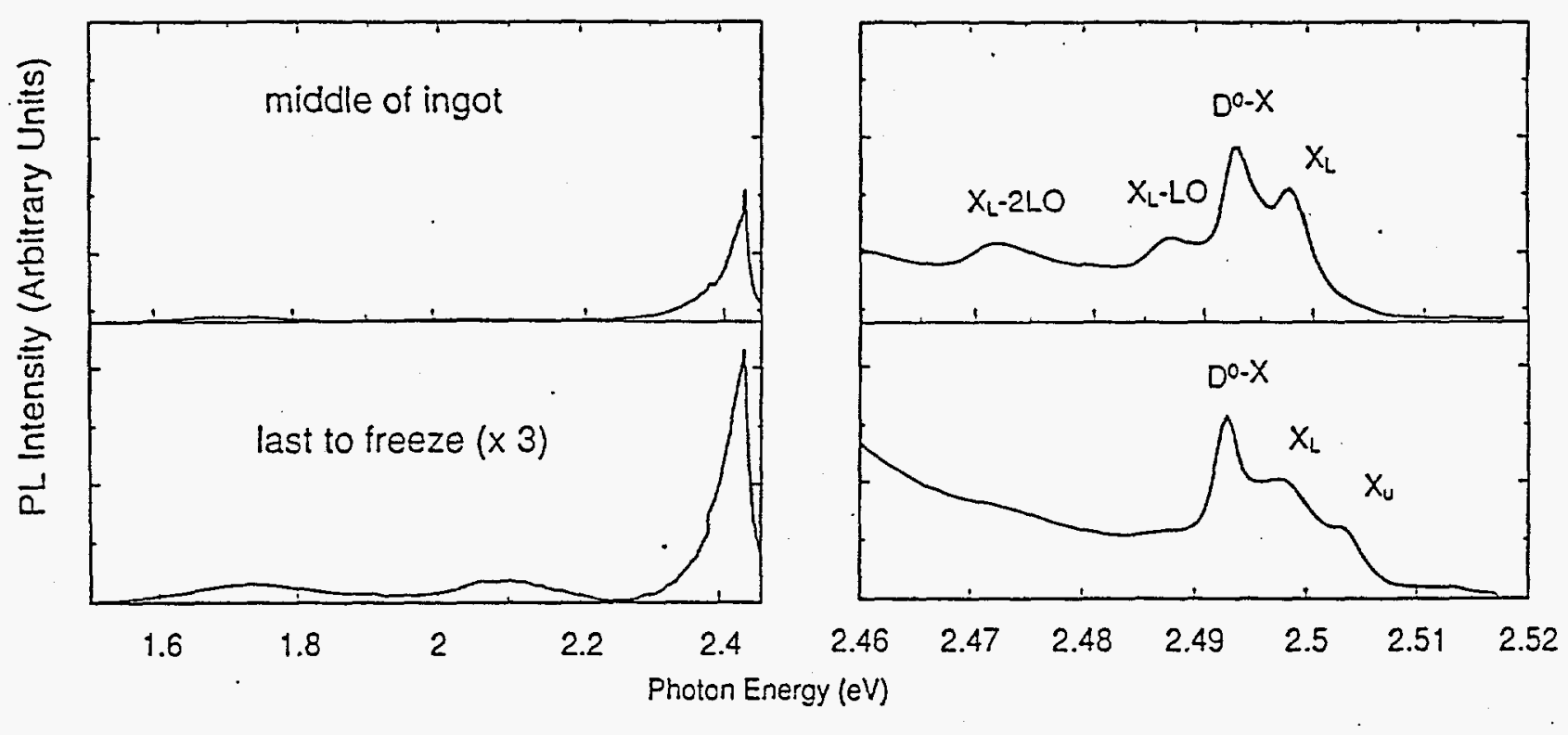


Figure 12. Triple axis x-ray diffraction of diamond saw cut $\mathrm{PbI}_{2}$.

\section{$\mathrm{Pbl}_{2}$ Cut by Diamond Saw \\ Triple Axis X-ray Diffraction (Reciprocal Space Map)}

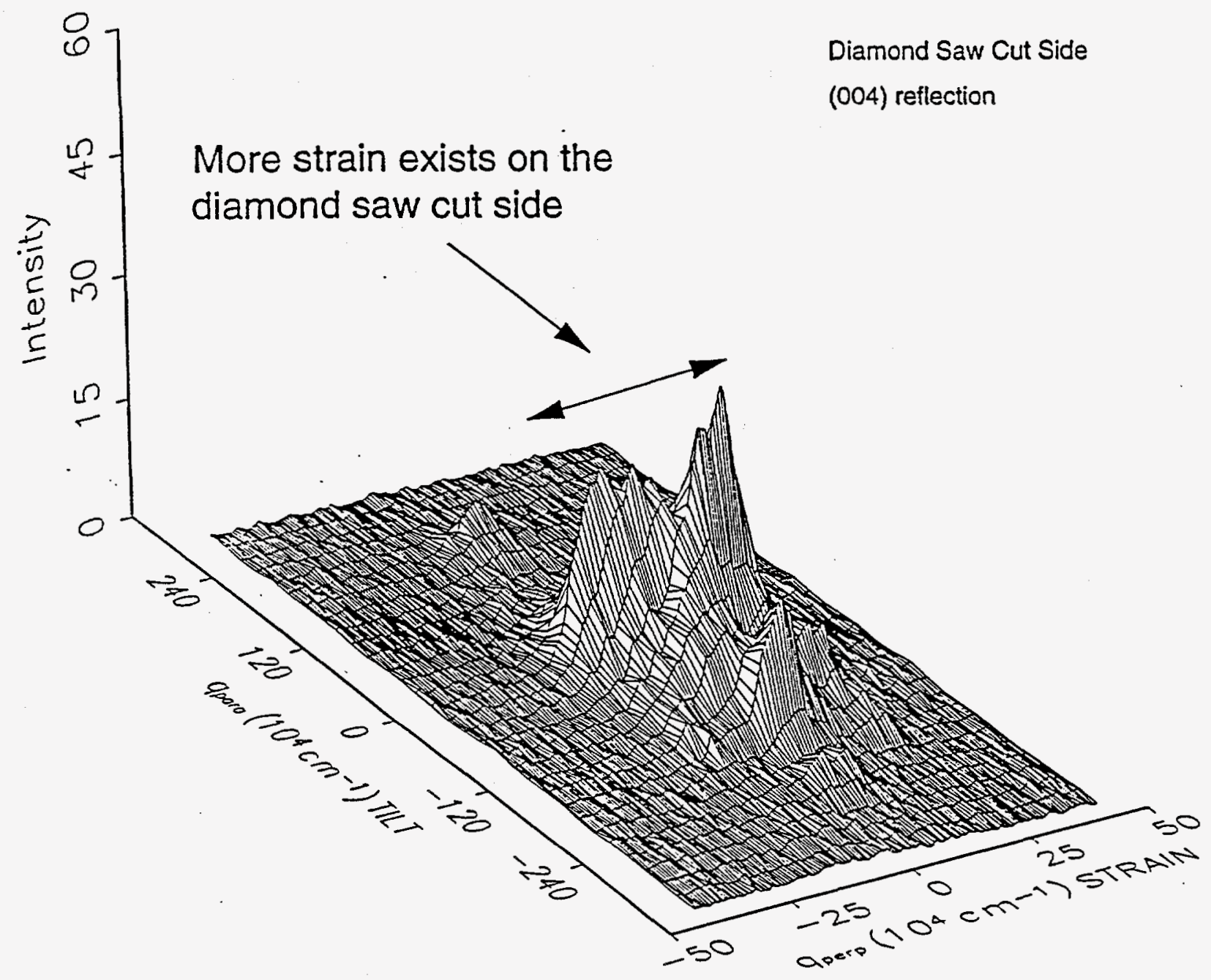


Figure 13. Triple axis $\mathrm{x}$-ray diffraction of diamond saw cut $\mathrm{PbI}_{2}$ after 1 minute etching.

\section{$\mathrm{Pbl}_{2}$ Cut by Diamond Saw \\ Triple Axis X-ray Diffraction (Reciprocal Space Map)}

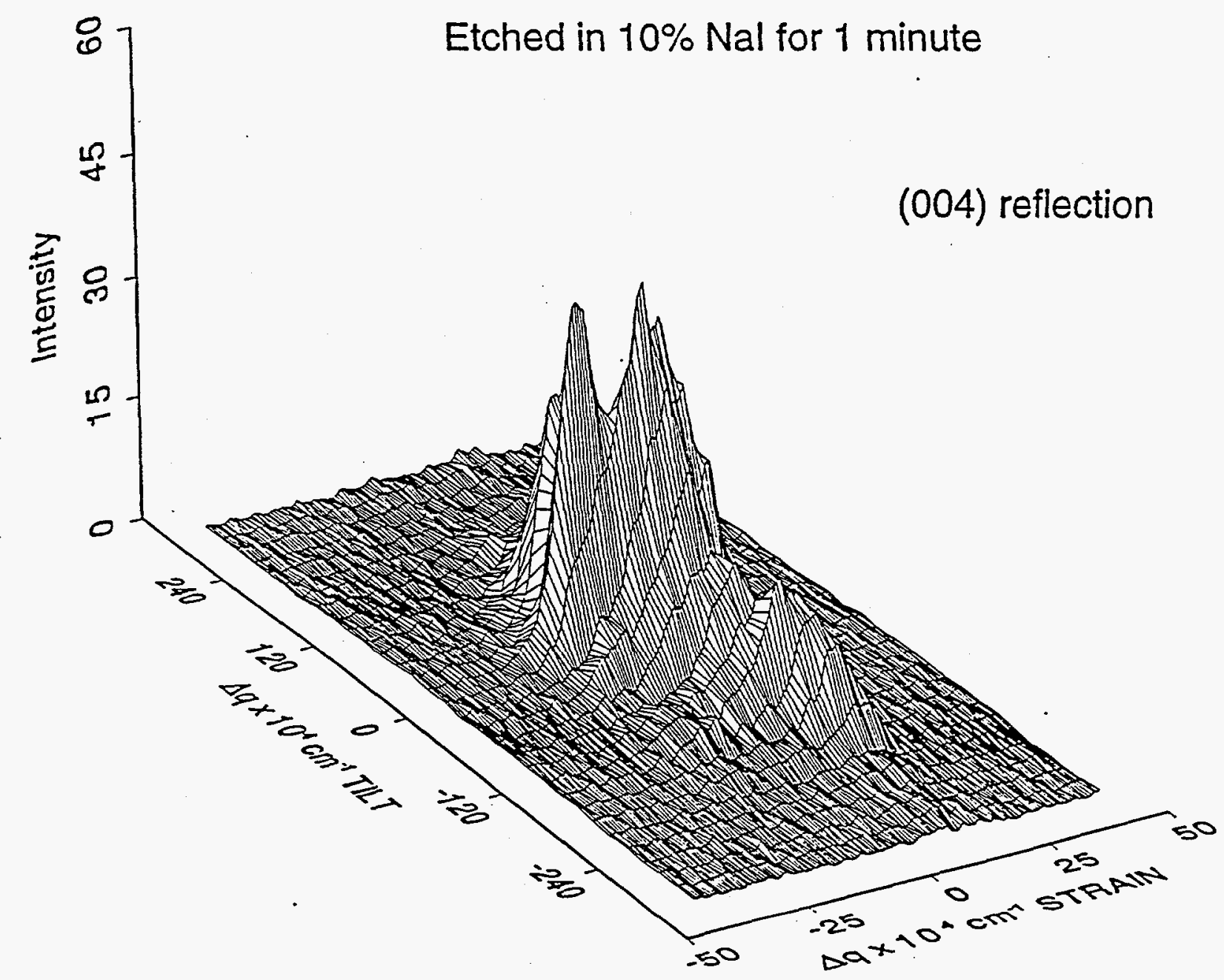


Figure 14. Triple axis $\mathrm{x}$-ray diffraction of diamond saw cut $\mathrm{PbI}_{2}$ after second etching.

\section{$\mathrm{Pbl}_{2}$ Cut by Diamond Saw \\ Triple Axis X-ray Diffraction (Reciprocal Space Map)}

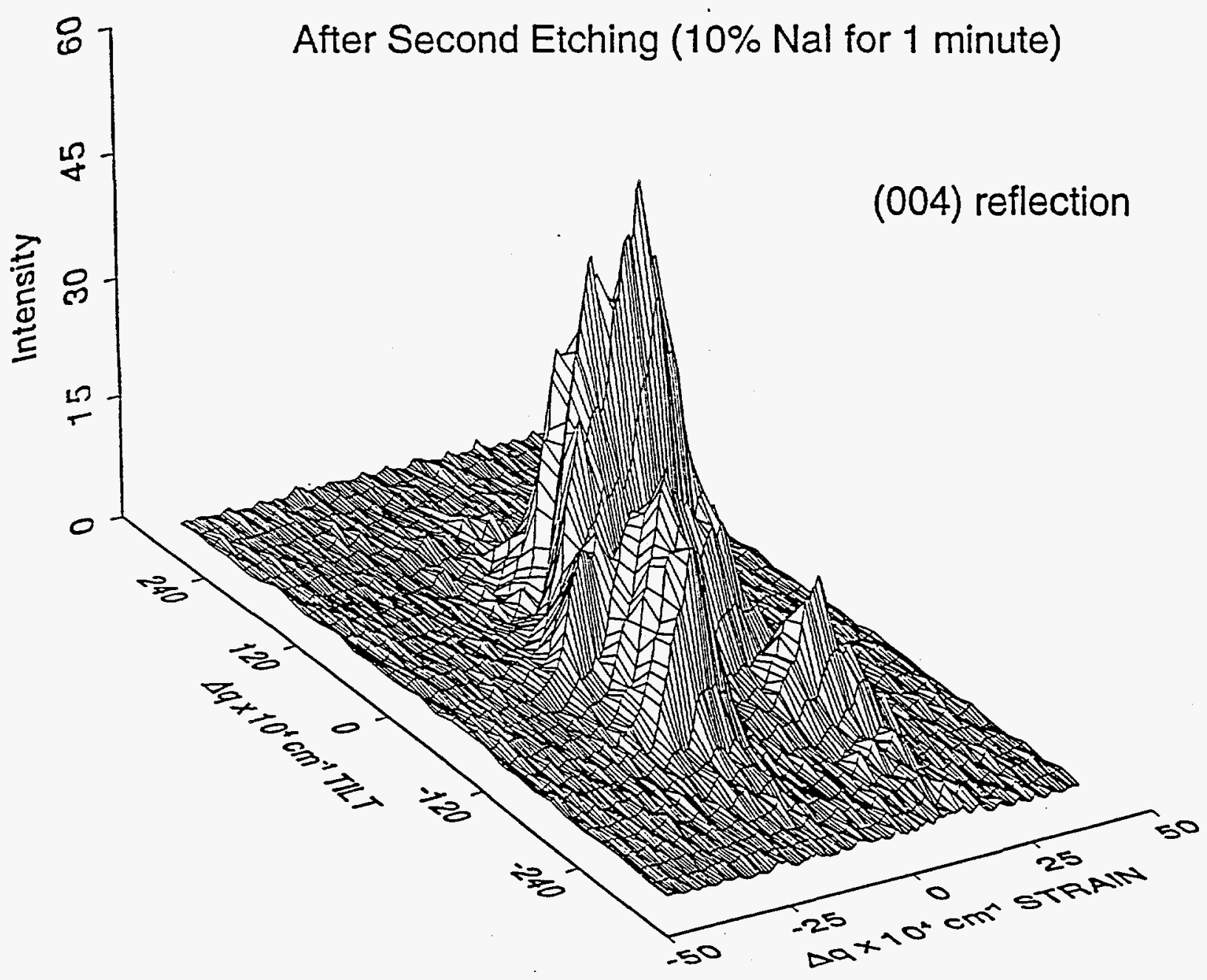


Figure 15. Triple axis $\mathrm{x}$-ray diffraction of thick $>1 \mathrm{~mm}$ cleaved sample.

\section{Triple Axis X-ray Diffraction (Reciprocal Space Map)}

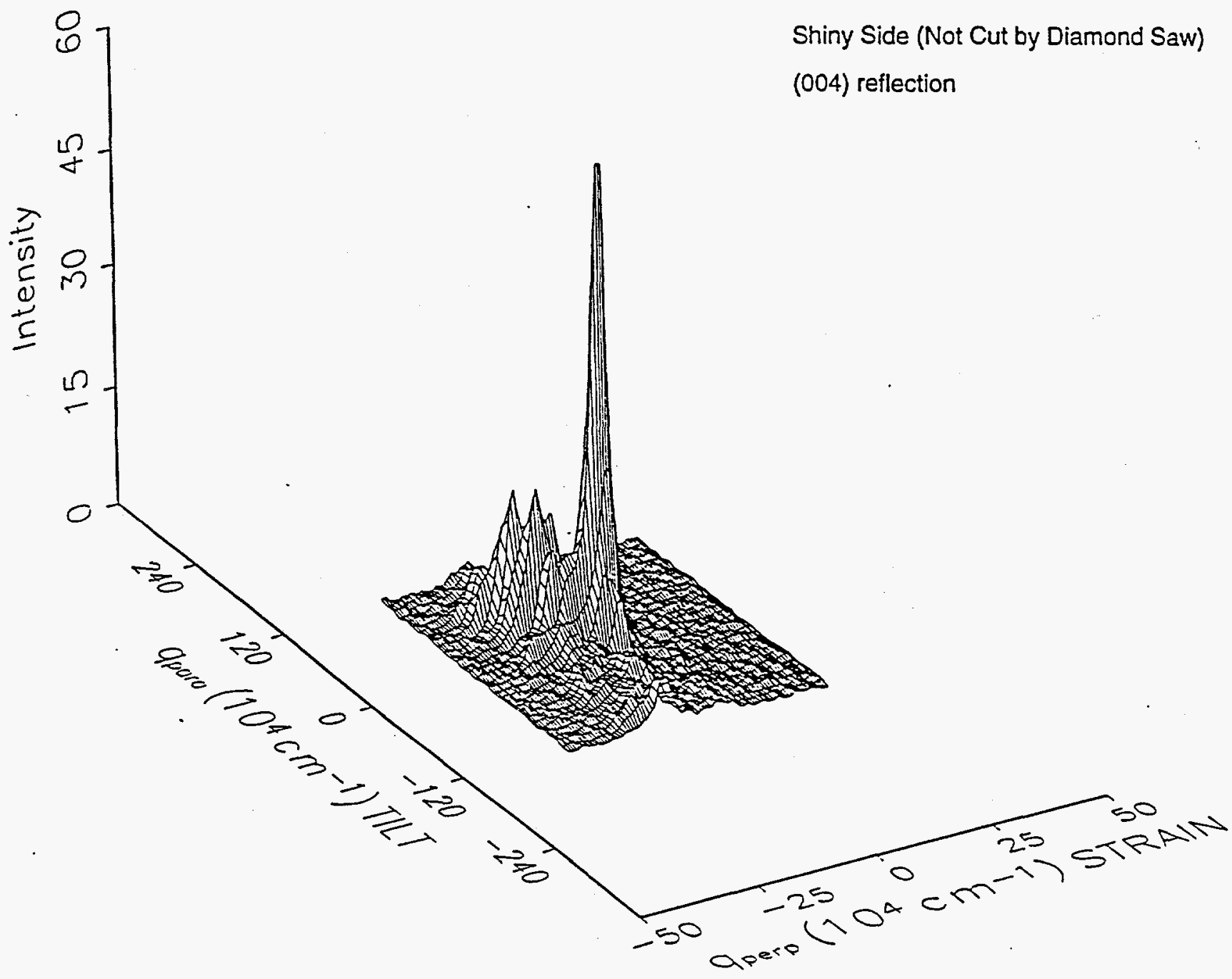


Figure 16. X-ray powder diffraction spectrum and identification of thin vs. thick diamond cut and etched samples. Note the better resolution on the thick sample.

\section{$\mathrm{Pbl} 2$ (RMD) \\ $\mathrm{X}$-ray Powder Diffraction Spectrum}

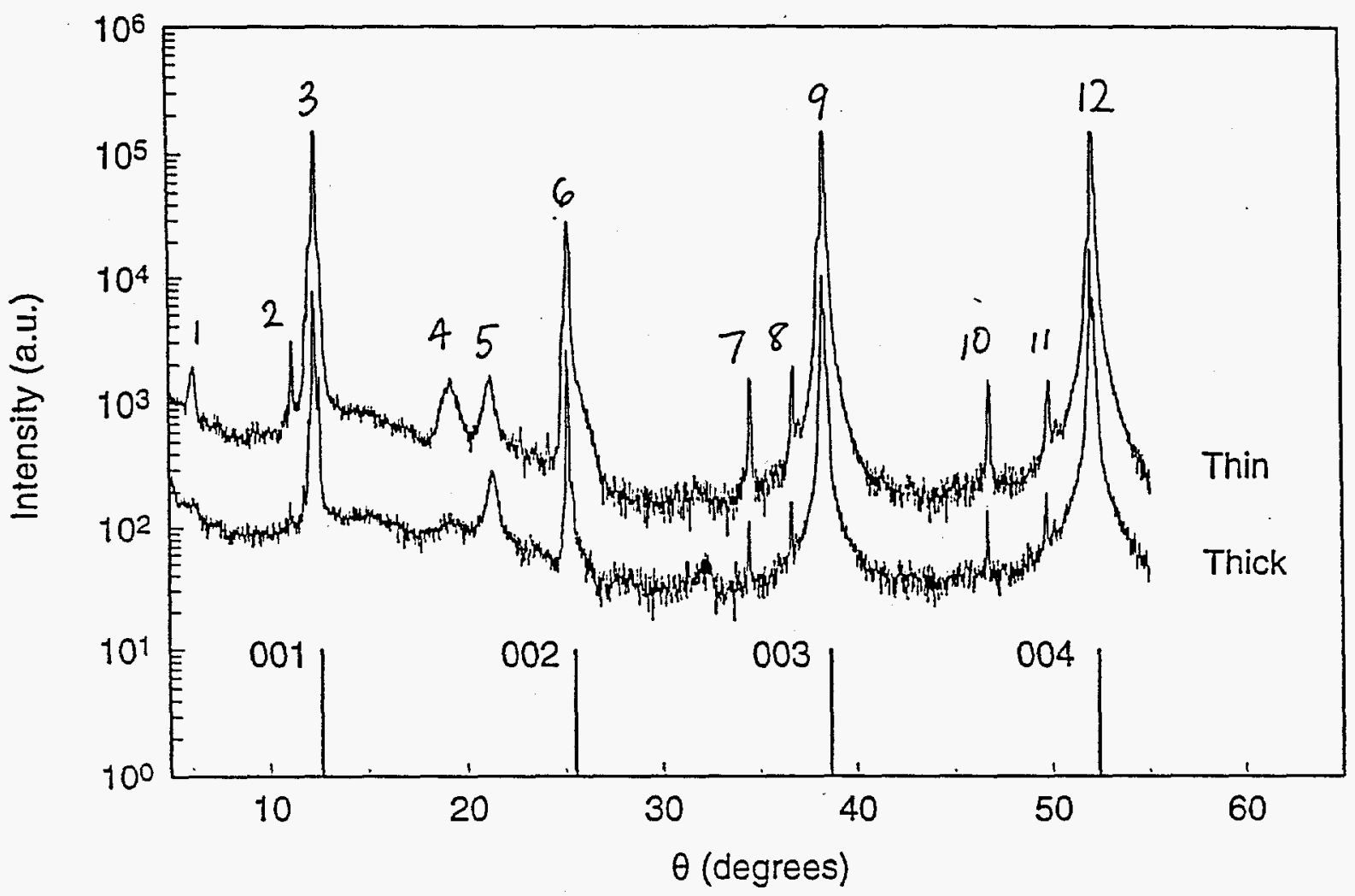

\begin{tabular}{|c|c|c|}
\hline Peak \# & 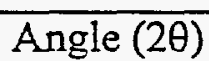 & Identification \\
\hline 1 & $6.20^{\circ}$ & (001) of " $4 \mathrm{H}$ " polytype \\
\hline 2 & $11.18^{\circ}$ & $\mathrm{CuK}_{\beta}$ \\
\hline 3 & $12.44^{\circ}$ & $(001)$ of "regular" $\mathrm{PbI}_{2}$ and/or (002) of " $4 \mathrm{H}$ " \\
\hline 4 & $19.14^{\circ}$ & $(003)$ of " $4 \mathrm{H}$ " \\
\hline 5 & $21.18^{\circ}$ & not yet identified \\
\hline 6 & $25.22^{\circ}$ & (002) of "regular" $\mathrm{PbI}_{2}$ and/or (004) of " $4 \mathrm{H} "$ \\
\hline 7 & $34.52^{\circ}$ & $\mathrm{CuK}_{\beta}$ \\
\hline 8 & $36.78^{\circ}$ & Tungsten line \\
\hline 9 & $38.42^{\circ}$ & (003) of "regular" $\mathrm{PbI}_{2}$ \\
\hline 10 & $46.72^{\circ}$ & $\mathrm{CuK}_{\mathrm{B}}$ \\
\hline 11 & $49.82^{\circ}$ & Tungsten line \\
\hline 12 & $52.08^{\circ}$ & (004) of "regular" $\mathrm{PbI}_{2}$ \\
\hline
\end{tabular}


Figure 17. High resolution $\mathrm{x}$-ray diffraction rocking curve on polished side vs. unpolished of $\mathrm{PbI}_{2}$ cut by diamond saw.

\section{Pbl2 Cut by Diamond Saw \\ High Resolution X-ray Diffraction Rocking Curve}

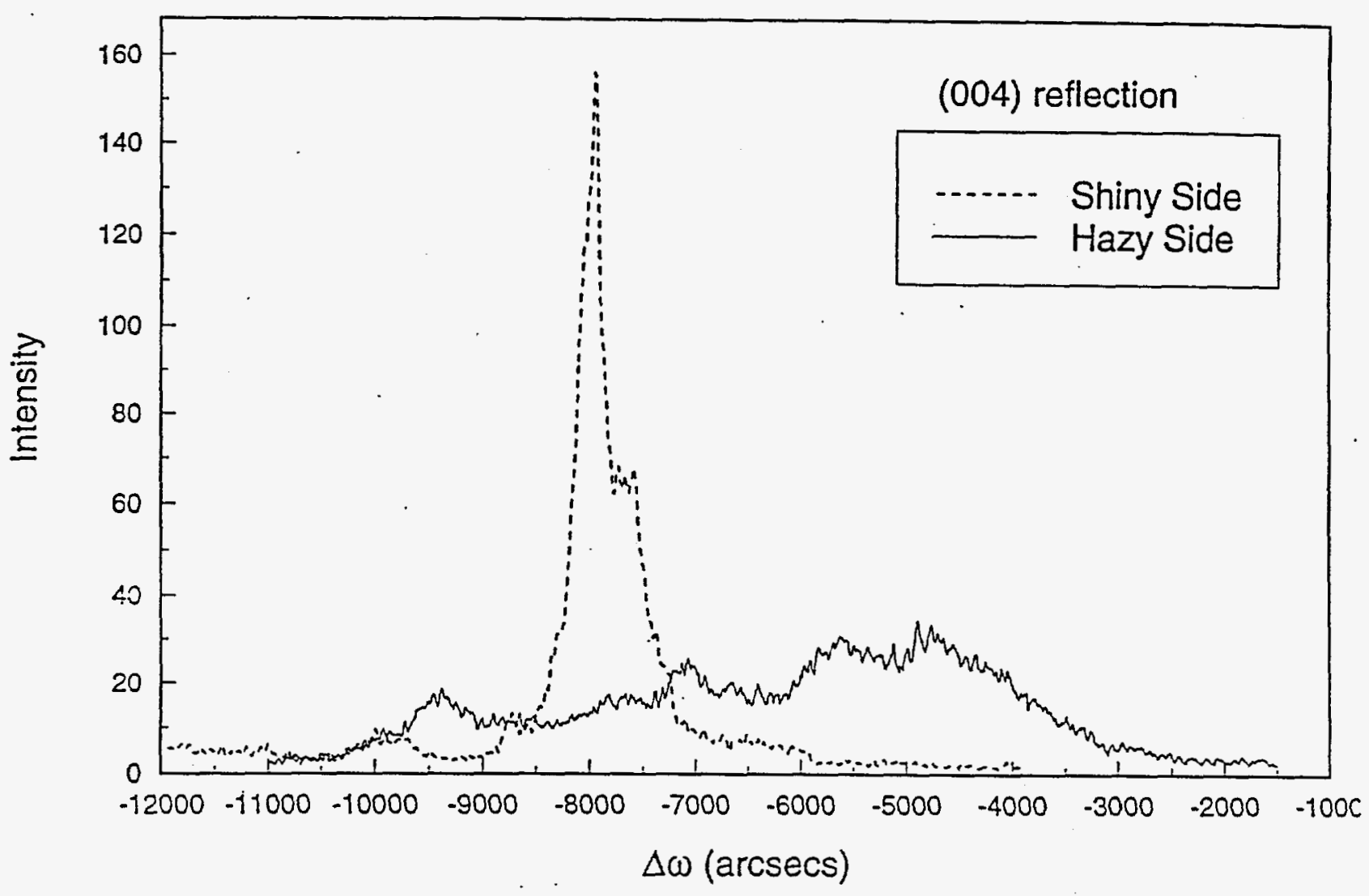


Figure 18. Surface structure of lead iodide after etching for 8 minutes. Left: lead iodide recrystallized on the surface.

Right: triangular etch pits.

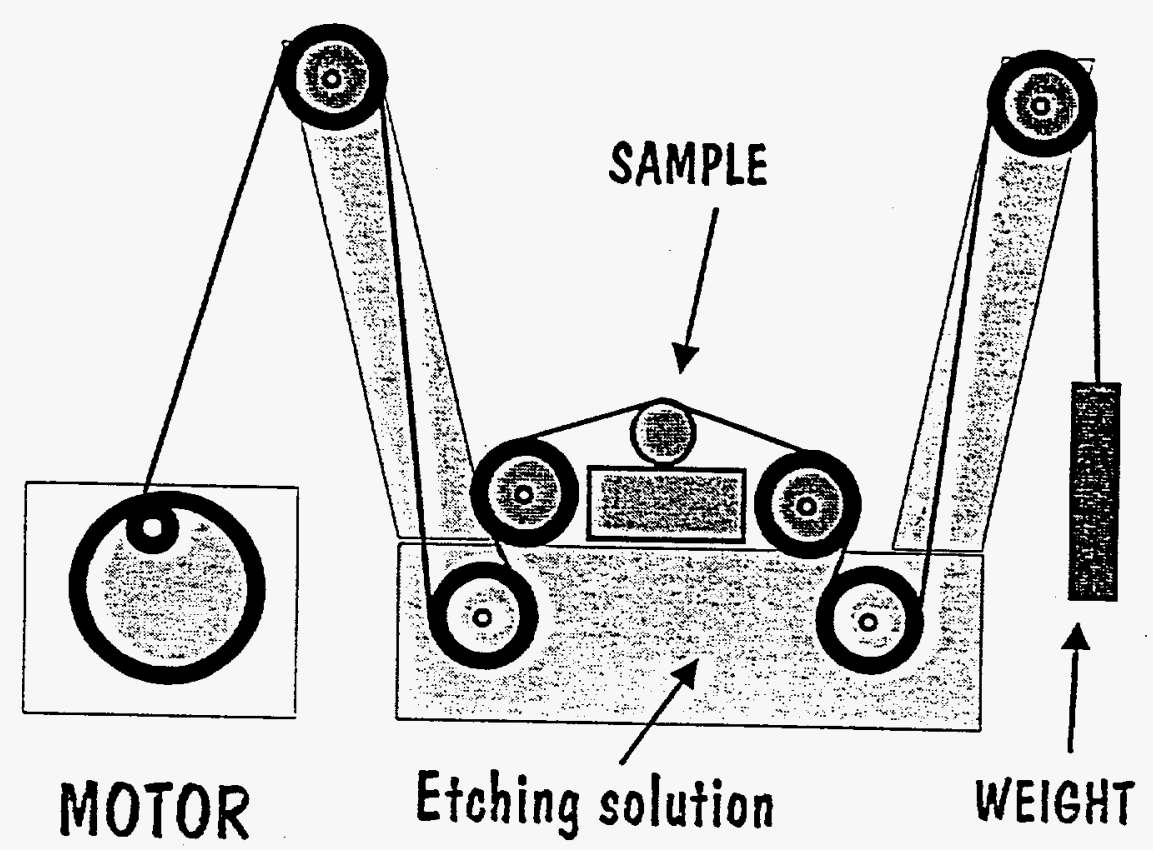


Figure 19. Carrier mobilities as functions of temperature for three values of mass anisotropies, $\sigma=1,2$ and 5 . Solid curves are for transport parallel to the $c$ axis, dotted lines are for transport perpendicular to the $c$ axis.
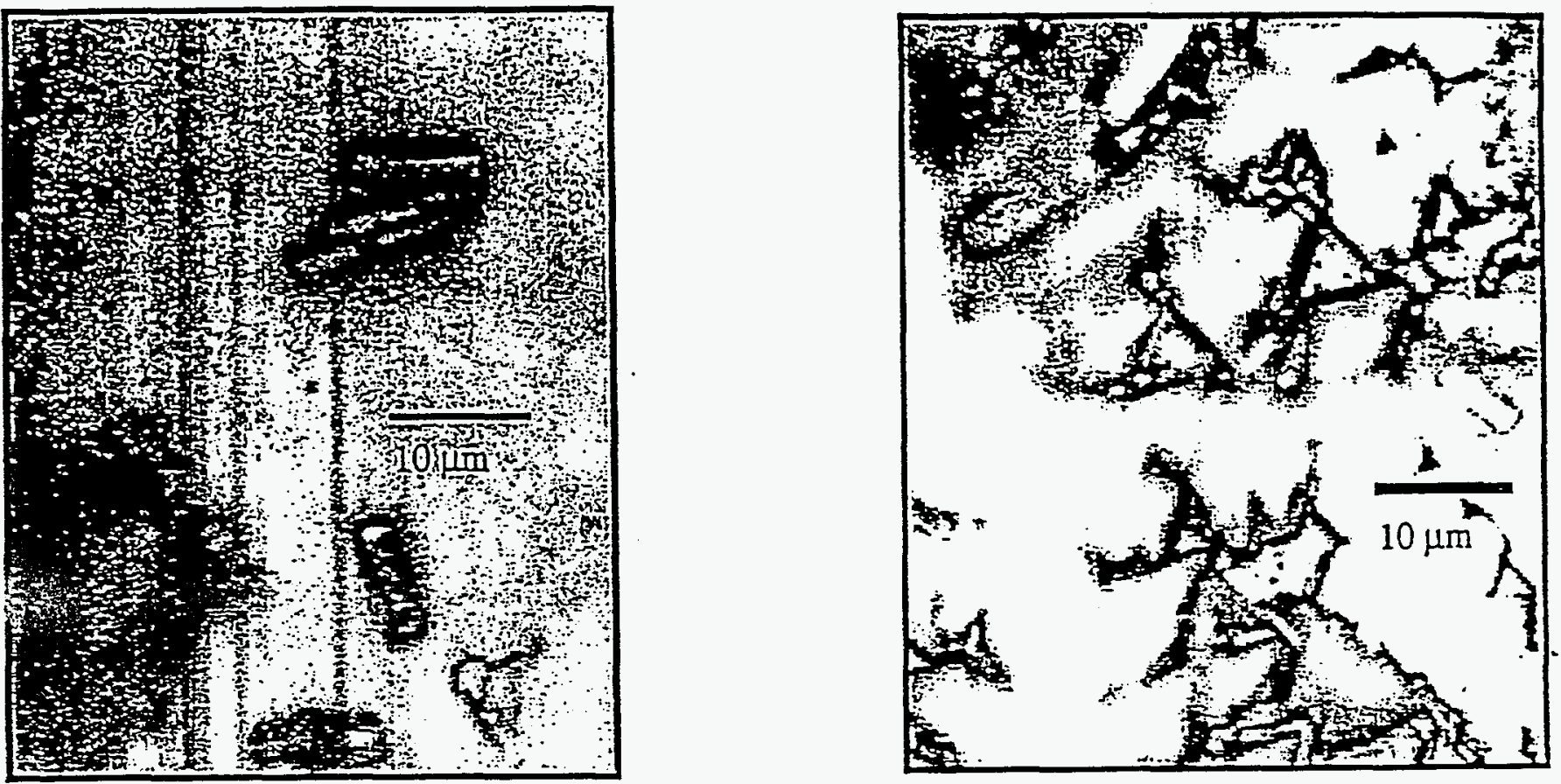
Figure 20. Chemical string saw used for $\mathrm{PbI}_{2}$.

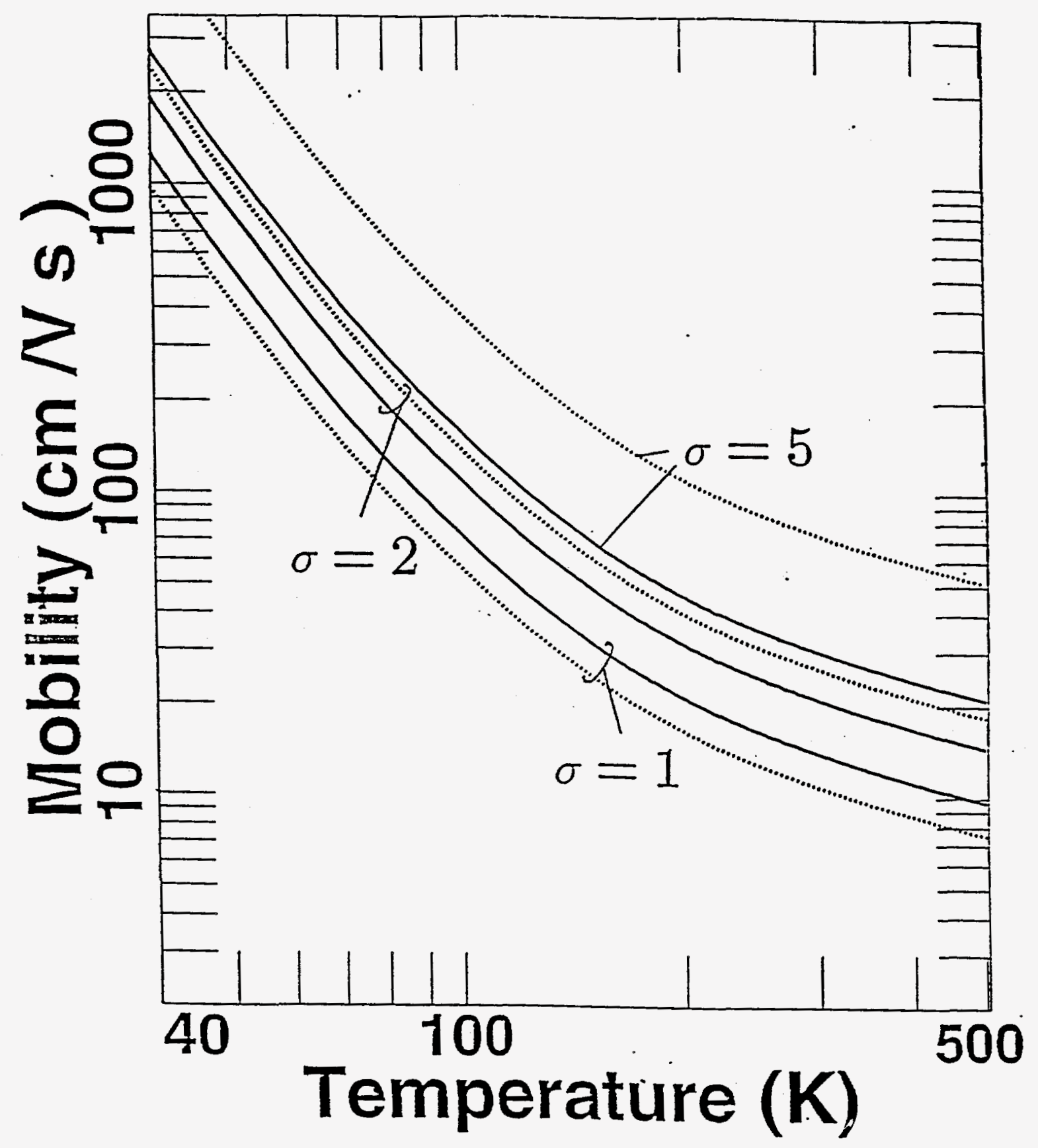


Figure 21. Spectrum of ${ }^{55} \mathrm{Fe}$ radiation measured by 100 micron thick $\mathrm{PbI}_{2}$.

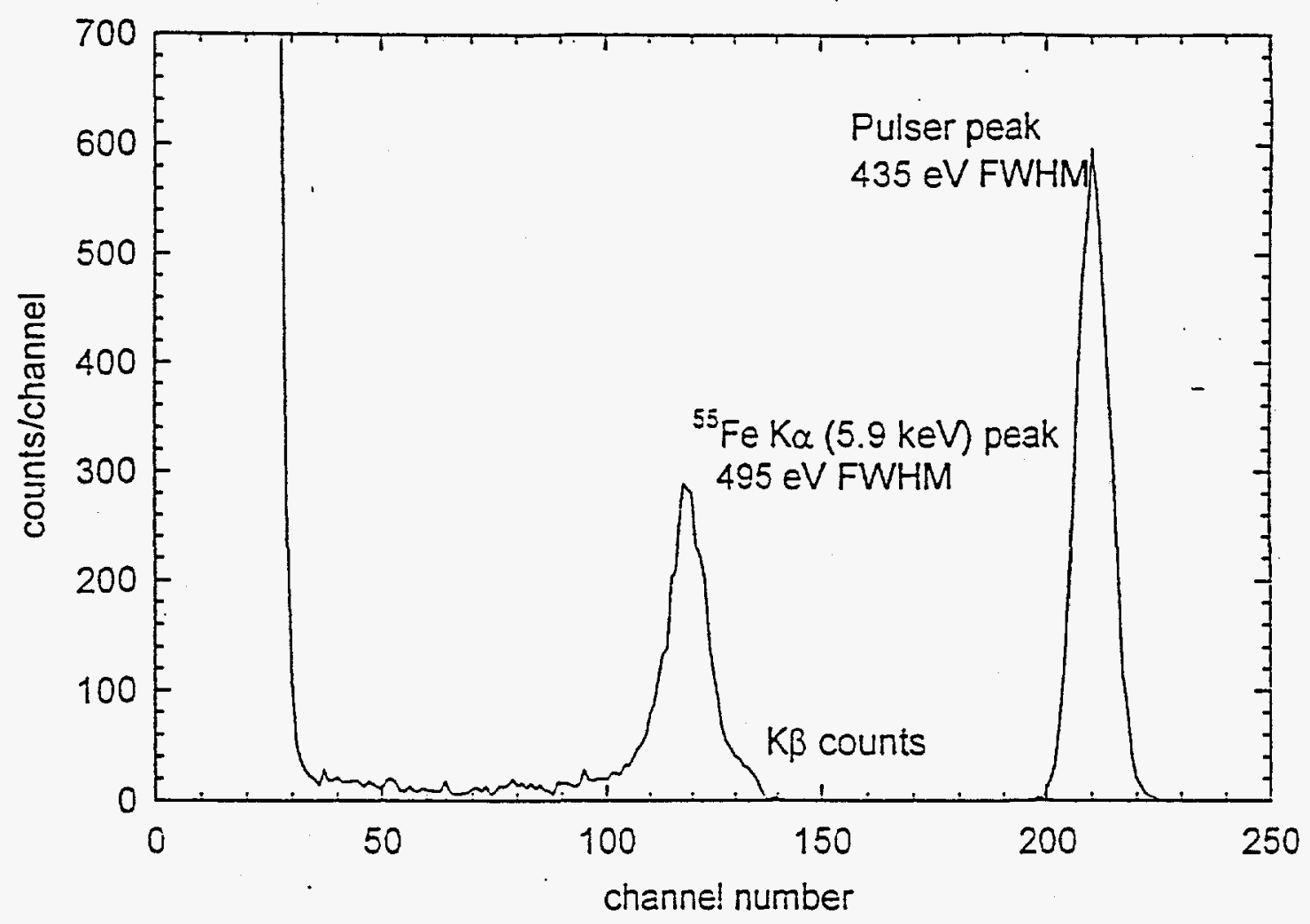




\title{
APPENDIX
}

Characterization of Lead Iodide for Nuclear Spectrometers

T.E. Schlesinger ${ }^{*}$, R.B. James ${ }^{*}$, M. Schieber ${ }^{*}$ J. Toney ${ }^{* *}$, J.M. Van Scyoc ${ }^{*}$, L. Salary", H. Hermon", J. Lund", A. Burger ${ }^{+}$, K.-T. Chen ${ }^{+}$, E. Cross", E. Soria", K. Shah ${ }^{++}$, M. Squillante ${ }^{++}$, H. Yoon ${ }^{++}$, M. Goorsky ${ }^{++}$

\author{
*Sandia National Laboratories, Livermore, CA, U.S.A. \\ ${ }^{* *}$ Carnegie Mellon University, Pittsburgh, PA, U.S.A. \\ 'Fisk University, Nashville, U.S.A. \\ ${ }^{+}$RMD Inc., Watertown, MA, U.S.A. \\ ${ }^{+++}$UCLA, Los Angeles, CA, U.S.A.
}

\begin{abstract}
We report on the results of a number of investigations into the material properties of lead iodide and their relation to $x$ and gamma ray spectrometers. The effectiveness of zone refining as determined by inductively coupled plasma optical emission spectroscopy is demonstrated. We show that zone refining is effective in producing lead iodide with a higher degree of purity in terms of extrinsic dopants and we determine the segregation coefficients for a number of these impurities. Low temperature photoluminescence also indicates an improvement in the material properties of the purified lead iodide. The chemical etching characteristics, including etch rates, of lead iodide is presented for a number of etching solutions. Triple axis $\mathrm{x}$-ray diffraction measurements have been employed to determine the structural perfection of the lead iodide after diamond sawing and etching and recovery of the crystallinity of the material is seen after $\mathrm{NaI}$ etching.
\end{abstract}




\section{Introduction}

Lead iodide, $\mathrm{PbI}_{2}$, was first introduced in the 1970s [1][2] as a candidate material for nuclear radiation spectrometry having an extraordinarily high photoelectric efficiency for gamma rays. In addition the wide band gap of this material $(2.55 \mathrm{eV})$ makes possible the growth of extremely high resistivity material and thus low background noise in nuclear detectors can be achieved. The high atomic numbers $(\mathrm{Pb}=82, \mathrm{I}=53)$ and density $\left(6.2 \mathrm{~g} / \mathrm{cm}^{3}\right)$ provide a high stopping power. Thus, room temperature, and even above room temperature, operation of gamma-ray spectrometers fabricated in this material is feasible.

Lead iodide consists of molecular layers $\mathrm{Pb}-\mathrm{I}-\mathrm{Pb}$, where the $\mathrm{Pb}$ ions are octahedrally coordinated [3] and where the bonding between the layers is of the molecular type, via van der Waals forces. Electrical measurement of melt-grown lead iodide single crystals have revealed a number of similarities between lead iodide and mercuric iodide, including resistivity greater than $10^{13}$ $\Omega \mathrm{cm}$ and a mean energy required to produce an electron-hole pair, $\varepsilon_{\mathrm{e}, \mathrm{h}}=6.49 \mathrm{eV}$ [4]. The room temperature drift mobilities of electrons and holes have also been reported to be 8 and $2 \mathrm{~cm}^{2} \mathrm{~V}^{-1}$ $\mathrm{s}^{-1}$, respectively [5]. The various polytypic phases of lead iodide have been extensively studied[3][6][7] and it has been established that high purity lead iodide is all of one polytype $(2 \mathrm{H})[6][8]$ with a melting point of $408^{\circ} \mathrm{C}$. It should be noted that the lack of any solid-solid phase transitions in lead iodide clearly distinguishes it from the related material mercuric iodide and allows it to be grown from the melt. In contrast, the destructive solid-solid phase transition between the orthorhombic and tetragonal phases of mercuric iodide at $130^{\circ} \mathrm{C}$ limits bulk detector grade mercuric iodide material to growth by vapor phase and this limits the growth rate compared to lead iodide.

Various methods for the growth of $\mathrm{PbI}_{2}$ single crystals have been evaluated. These include the Czochralski method, vapor phase growth, gel growth, and Bridgman growth. The Bridgman crystal growth method is perhaps the simplest melt based technique, and it has been used extensively and successfully for the growth of lead iodide crystals for detector applications. High quality melt-grown lead iodide crystals have became available recently and $x$-ray and gamma ray radiation detectors have been fabricated from these[9][10][11][12][13]. The primary difference between recently demonstrated lead iodide detectors and those fabricated earlier appears to be the 
degree of crystal purity[1][2]. Crystallinity and orientation are most likely critical in determining detector performance. In addition the fabrication process of lead iodide $x$-ray and gamma ray detectors consists of several steps: extraction of slabs from crystals (sawing), surface preparation (etching), deposition of electrical contacts, and encapsulation[15]. In this paper we discuss a number of material characterization results related directly to the fabrication of $\mathrm{PbI}_{2} \mathrm{x}$-ray and gamma-ray detectors including zone refining, chemical etching, photoluminescence characterization, and triple axis $\mathrm{x}$-ray diffraction.

\section{Zone Refining and Impurity Analysis}

Purification of commercially available lead iodide material is necessary because even the best available material (99.9999\%) has a significant concentration of impurities by semiconductor standards. These can cause high carrier trapping and therefore low carrier lifetime in the resulting detectors. The zone purification[14] process has been used as a purification method for materials that melt congruently. In particular zone refining has been investigated as a means of obtaining $\mathrm{PbI}_{2}$ with reduced extrinsic defect concentrations. The technique is implemented by melting a portion of the material and this melted zone is then slowly passed through the solid material. The expected result is a charge much purer at the middle section than the end sections. One important parameter in zone refining is the segregation coefficient: the ratio of the solubility of the impurity in the solid to the solubility in the melt. Since most impurities are more soluble in the liquid phase than in the solid phase they tend to accumulate in the liquid phase. The number of zone refining passes is also important to the effectiveness of purification. Attempts to purify lead iodide starting materials by zone refining to produce a reasonable large amount of high purity starting materials for crystal growth have been reported [10][11][12][13], and the results showed that the detector performance could be improved for detectors fabricated from the section of the charge consisting of what would be expected to be the purest material. To quantitatively assess the effectiveness of zone refining as a means of purification of $\mathrm{PbI}_{2}$ we have employed inductively coupled plasma optical emission spectroscopy (ICPOES) to investigate the presence and concentrations of impurities in zone refined $\mathrm{PbI}_{2}$. We have employed a computer controlled zone refining furnace for purifying lead iodide. Two heater rings were used to increase the process effi- 
ciency. The process involves loading commercially available lead iodide powder in a quartz ampoule and evacuating the ampoule to about $50 \mu \mathrm{m} \mathrm{Hg}$ pressure. The ampoule is then sealed and the $\mathrm{PbI}_{2}$ powder is melted to form a solid ingot. The ampoule is mounted onto the zone refining furnace, the heaters set to a temperature of $500^{\circ} \mathrm{C}$ (which exceeds the melting point of $\mathrm{PbI}_{2}$ of $408^{\circ} \mathrm{C}$ ), and the heaters moved at a speed of about $2 \mathrm{~cm} / \mathrm{hr}$ down the length of the ingot. After each pass the heater is moved quickly back in the reverse direction at about $20 \mathrm{~cm} / \mathrm{hr}$ and this prevents melting of the ingot during the reverse motion and hence prevents impurities from being transported back through the ingot. A large number $(\sim 100)$ of passes are carried out in this manner.

For the ICPOES measurements $4 \mathrm{gm}$ (six significant figures) samples were immersed in $8 \mathrm{ml}$. of aqua regia in a tefion digestion sample and left overnight. All reagents were J.T. Baker Ultra pure. After dilution with deionized water (approx.50 ml) the samples were placed in a CEM 81-D microwave oven and open digested for forty minutes at $30 \%$ power. A closed vessel digestion was then performed at $50 \%$ power for 40 minutes. Samples were then cooled and transferred to a 100 ml. polypropylene nalgene volumetric fiask with the balance of the volume made up of $10 \%$ aqua regia. The sample was divided into two samples with $400 \mathrm{ppb}$ spike solution (SPEX Industries ICPMS-2) added to one sample and a similar volume of $(2 \mathrm{ml})$ of $10 \%$ aqua regia added to the other. Impurity levels were determined using a Perkin Elmer P-100 Sequential ICPMS and a Cetac U-500 Ultra Nebulizer. Estimated detection limits for most elements was below 1 ppb with the exception of $\mathrm{Cu}$ and $\mathrm{Fe}$ at about $2 \mathrm{ppb}$ and $\mathrm{Ni}$ at about $7 \mathrm{ppb}$.

Samples from the bottom, middle, and upper sections of the zone refined ingot were analyzed and the concentrations of a number of impurities ( $\mathrm{Ca}, \mathrm{Na}, \mathrm{Fe}, \mathrm{Zn}, \mathrm{Al}, \mathrm{Cu}, \mathrm{Mg}, \mathrm{Ni}, \mathrm{Ag}, \mathrm{Mn}, \mathrm{Cr}$ ) were determined. Impurity concentrations for these elements ranges below about $10 \mathrm{ppm}$ and there is a strong trend of impurities segregating to the bottom of the ingot as a consequence of the zone refining. It also appears that the total impurity concentration is a minimum towards the middle of the ingot indicating that for some elements the segregation coefficient is near or above unity and this is presented in Figure 1. Figure 2 shows the concentration profiles for $\mathrm{Mn}, \mathrm{Ag}$, and $\mathrm{Cu}$. For these elements the concentration decreases to a value below the detection limit for this technique and clearly indicates that the zone refining method is very effective. Using this data we have 
computed the segregation coefficient for a number of elements and these are summarized in Table 1. These segregation coefficients were arrived at using existing models for impurity concentrations in a zone refining process as shown in Figure 3 for $\mathrm{Mn}$ as an example. The model assumes an impurity concentration which follows the form, $C(x)=A \exp (B x)$, where $x$ is the distance along the ingot and $A$ and $B$ are constants. The effective segregation coefficient, $k_{\text {eff }}$ is calculated using $k_{\text {eff }}=B L(\exp (B l)-l)$, where $l$ is the zone length. The constant $A$ is proportional to the initial uniform impurity concentration $C_{0}$ and is expressed as $A=C_{0} B L /(\exp (B L-I)$, where $L$ is the ingot length. In view of the good agreement between the model and this data we expect that these estimates are reasonably accurate.

\section{Photoluminescence}

Low temperature photoluminescence (PL) spectroscopy was performed on lead iodide samples taken from the middle and last to freeze sections of a zone refined ingot. The PL spectra were taken with the samples maintained at $4.2 \mathrm{~K}$ in a low temperature immersion cryostat. The excitation source was an argon ion laser tuned to the $454 \mathrm{~nm}$ line and set at a power level of $40 \mathrm{~mW}$. The laser excitation source was chopped and not focussed onto the surface of the sample to avoid excessive excitation power densities. The luminescence was collected from the front surface of the sample and directed to the entrance slits of a SPEX model $14043 / 4 \mathrm{~m}$ spectrometer set to a resolution of about $2 \AA$. It was then detected by a PMT with an $S 20$ response and associated computer controlled data acquisition system. The photoluminescence spectrum which is characteristic of the $2 \mathrm{H}$ polytype may be generally divided into two regions, the near band edge luminescence $(E=2.52 \mathrm{eV}-2.25 \mathrm{eV})$ and the deeper region $(E<2.25 \mathrm{eV})$. The spectra are presented in Figure 4 with some of the features labeled according the identification of Brodin et al[16] and a number of observations may be made regarding the observed spectra. The intensity of the near-band-edge luminescence is about a factor of three greater for the spectra obtained from the sample from the middle of the ingot indicating an overall higher quality material. An increase in this near-bandedge luminescence is probably an indication of a lower concentration of nonradiative or deep recombination centers that could be associated with impurities and defects. In the near band edge region of the spectrum the features are also more well resolved again indicating higher quality 
material. The lower energy luminescence is dominated by two bands centered at about $2.10 \mathrm{eV}$ and $1.72 \mathrm{eV}$ which appear to be somewhat more intense, relative to the near band edge luminescence, in the spectra taken from the last to freeze samples indicating, perhaps, a greater concentration of deeper radiative recombination centers in this material. All these observations are consistent with the trend observed in the ICPOEMS studies, namely, that the middle of the ingot tends to be freer of extrinsic impurities.

\section{Chemical Etching}

A key step in the fabrication of nuclear spectrometers is the preparation of the semiconductor surface before the deposition of contacts. This surface (and near surface region) should be smooth, defect and trap free so as to ensure that the recombination rate for carriers is not unusually great. Potassium Iodide (KI), for example, is an effective etchant commonly used as a $10 \%$ solution in deionized water in the processing of $\mathrm{HgI}_{2}$. This solution has been considered and found not to be effective in etching $\mathrm{PbI}_{2}$ giving slow or not reproducible etch rates. Thus we have explored the use of a number of other etching solutions including Sodium Iodide (NaI) and Sodium Thiosulfate $\left(\mathrm{Na}_{2} \mathrm{~S}_{2} \mathrm{O}_{3}\right)$. Etching experiments were performed by polishing a razor cleaved or diamond sawn wafer of $\mathrm{PbI}_{2}$ for a fixed amount of time on a cloth saturated with the etching solution and followed by a deionized water rinse. From this a linear etch rate was inferred. Alternatively samples were immersed in etching solution and their weight loss measured after a fixed period of time and a volume etch rate was calculated. The results are summarized in table 2. No significant differences were observed in terms of etching characteristics between samples that were razor cleaved or diamond sawn. At this time a $10 \% \mathrm{NaI}$ solution appears to be the most controlled etch. This solution is capable of removing some surface damage for short etch times. However at longer times triangular etch pits become clearly visible on the surface as does redeposited material. This redeposited material appears to be insoluble in these solutions and thus one of these etch solutions appear capable of providing a surface suitable for contact deposition. It appears that further investigation of these solutions at other temperatures or concentrations may yield improved etching characteristics.

Triple axis $\mathrm{x}$-ray measurements of diamond sawn lead iodide show the near surface region 
$(<10 \mu \mathrm{m})$ of the material to be significantly damaged. The overall reflected $x$-ray intensity is reduced and the line width is quite broad. However, after approximately two to five minutes of etching with gentle agitation in a $10 \%$ by weight $\mathrm{NaI}$ solution a significant improvement in the triple axis $\mathrm{x}$-ray reciprocal space map is seen. The intensities of the reflected peaks are increased and the linewidth of these features are decreased, both indicating an improved crystallinity in the near surface region. After a longer etch time, which removes all surface damage due to diamond sawing, triple axis $x$-ray diffraction measurements revealed no plastic deformation induced in the bulk ( $>1 \mathrm{~mm}$ ) in contrast to cleaved or tape pealed slices which do show plastic deformation. Thus we conclude that chemical etching can be used to controllably remove the damaged near surface region that results from diamond sawing of lead iodide. The performance of spectrometers fabricated from improved material has been evaluated and pulse height spectra obtained for both ${ }^{55} \mathrm{Fe}$ and ${ }^{241} \mathrm{Am}$ and these have been reported elsewhere[15].

\section{Conclusions}

In conclusion we have shown that zone refining is effective in reducing the concentrations of many impurities in lead iodide. In addition we have obtained the segregation coefficients for a number of these impurities. The material quality is thus improved as seen in low temperature PL measurements and defects at the surface can be minimized with the appropriate chemical etch. With the appropriate processing techniques it has been found that detectors fabricated from high purity crystal exhibit significant improvement in performance compared to those produced from low purity crystals. However, problems still exists in lead iodide due to low charge carrier collection efficiency and this low efficiency is probably caused by additional impurities or defects incorporated during crystal growth and detector fabrication processes. In addition we are currently investigating the use of pulse processing techniques to improve energy resolution and detector efficiency. 


\section{References}

1. S. Roth and W. R Willig, Appl. Phys. Lett. 18, 328(1971).

2. C. Manfredotti, R. Murri, A. Quirini, and L. Vasanelli, IEEE Trans. Nuc. Sci. NS-24, 126(1977).

3. B. Palosz, Phys. Stat. Sol. (a) $80,11(1983)$.

4. F. Olschner, J. C. Lund, K. S. Shah, and M. R. Squillante, ICFA Instrum. Bull. 7, 9(1989).

5. R. Minder, G. Ottaviani, and C. Canali, J. Phys. Chem. Solids xx, 417(1976).

6. M. Rao and O. N. Srivastava, J. Phys. D: Appl. Phys. 11, 919(1978).

7. S. K. Chaudhary and G. C. Trigunayat, J. Crystal Growth 62, 398(1983).

8. M. Chand and G. C. Trigunayat, J. Crystal Growth 39, 299(1977).

9. J. C. Lund, K. S. Shah, M. R. Squillante, and F. Sinclair, IEEE Trans. Nuc. Sci. NS-35, 89(1988).

10. J. C. Lund, K. S. Shah, M. R. Squillante, L. P. Moy, F. Sinclair, and G. Entine, Nucl. Inst. and Meth. A283, 299(1989).

11. J. C. Lund, K. S. Shah, F. Olschner, J. Zhang, L. P. Moy, F. Medrick, and M. R. Squillante, Nucl. Inst. and Meth. A322, 464(1992).

12. J. Zhang, K. S. Shah, F. Olschner, J. C. Lund, L. P. Moy, K. Daley, L. Cirignano, and M. R. Squillante, Nucl. Inst. and Meth. A322, 499(1992).

13. D. C. Dominique, R. B. James, H. Feemster, R. Anderson, A. J. Antolak, D. H. Morse, A. E. Pontau, H. Jayatirtha, A. Burger, X. J. Bao, T. E. Schlesinger, G. S. Bench, and D. W. Heikkinen, Mat. Res. Symp. Proc. 302, 335(1993).

14. W. G. Pfann, “Zone Refining”, Robert E. Kreiger Publishing Co., Huntington, New York (1978).

15. J. C. Lund, F. Olschner, and A. Burger, Ch. 11, in Volume 43 of Semiconductors and Semimetals, "Semiconductors for Room Temperature Nuclear Detector Applications", editors: T.E. Schlesinger and R. B. James, Academic Press, New York, (1995).

16. M.S. Brodin, A.O. Gushcha, L.V. Taranenko, V.V. Tishchenko, V.N. Khotyaintsev, S.G. Shevel, Sov. Phys. Solid State 28, 1658(1986). 
Table 1: Elemental Segregation Coefficients $k_{\text {eff }}$

\begin{tabular}{|l|c|}
\hline Element & $\mathrm{k}_{\text {eff }}$ \\
\hline \hline Chromium & 0.84 \\
\hline Manganese & 0.71 \\
\hline Silver & 0.66 \\
\hline Iron & 0.65 \\
\hline Copper & 0.59 \\
\hline Calcium & 0.55 \\
\hline
\end{tabular}


Table 2: Etching Rates for $\mathrm{PbI}_{2}$

\begin{tabular}{|l|l|l|}
\hline \multicolumn{1}{|c|}{ Etching Solution } & Etch Rate & \multicolumn{1}{|c|}{ Comments } \\
\hline \hline $\begin{array}{l}\text { Saturated NaI } 66 \% \\
\text { by wt.) }\end{array}$ & $100 \mu \mathrm{m} / \mathrm{min}$ & reaction rate too great \\
\hline $25 \% \mathrm{NaI}$ by wt. & $80 \mu \mathrm{m} / \mathrm{min}$ & considerable surface damage and roughening \\
\hline $10 \% \mathrm{NaI}$ by wt. & $10 \mu \mathrm{m} / \mathrm{min}$ & $\begin{array}{l}\text { some surface damage removed at short times, however, } \\
\text { for etching times greater than 10 minutes surface } \\
\text { becomes rough }\end{array}$ \\
\hline $\begin{array}{l}10 \% \mathrm{Na}_{2} \mathrm{~S}_{2} \mathrm{O}_{3} \\
\text { by wt. }\end{array}$ & - & rapid reaction causing sample to flake apart \\
\hline $10 \% \mathrm{KI}$ by wt. & - & Rates not reproducible \\
\hline
\end{tabular}


Figure 1. Total impurity concentration measured in a zone refined lead iodide ingot as measured by ICPOEMS. While it is observed that most impurities segregate to the bottom (last to freeze) section of the ingot, some impurities segregate to the upper portion of the ingot and thus the center of the ingot may be the most pure.

\section{Total Impurity Concentration}

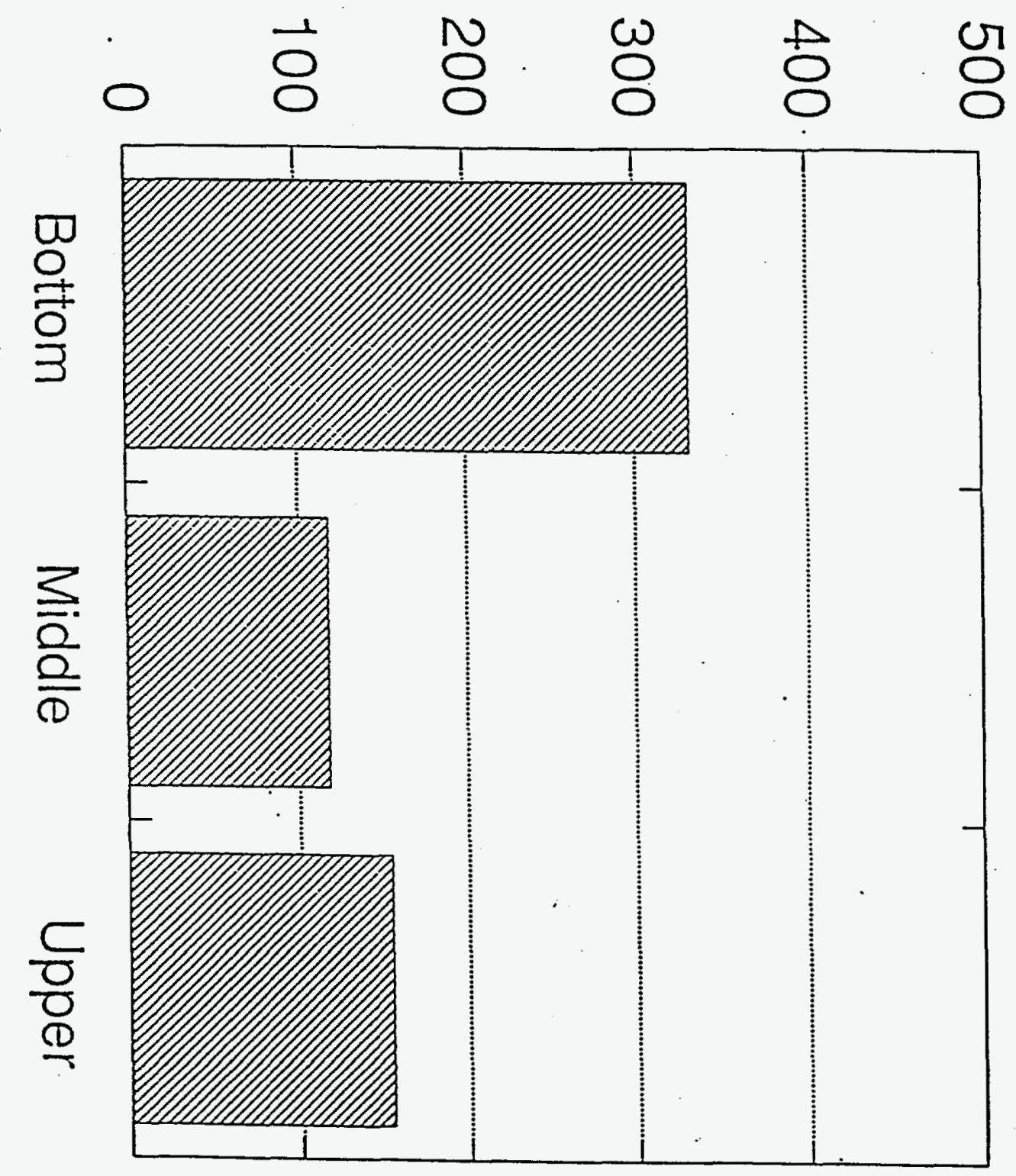


Figure 2. Impurity concentration of $\mathrm{Mn}, \mathrm{Cu}$, and $\mathrm{Ag}$ as obtained by ICPOEMS for a zone refined lead iodide ingot. From this and similar data, the segregation coefficients for a number of elements were obtained in this study.

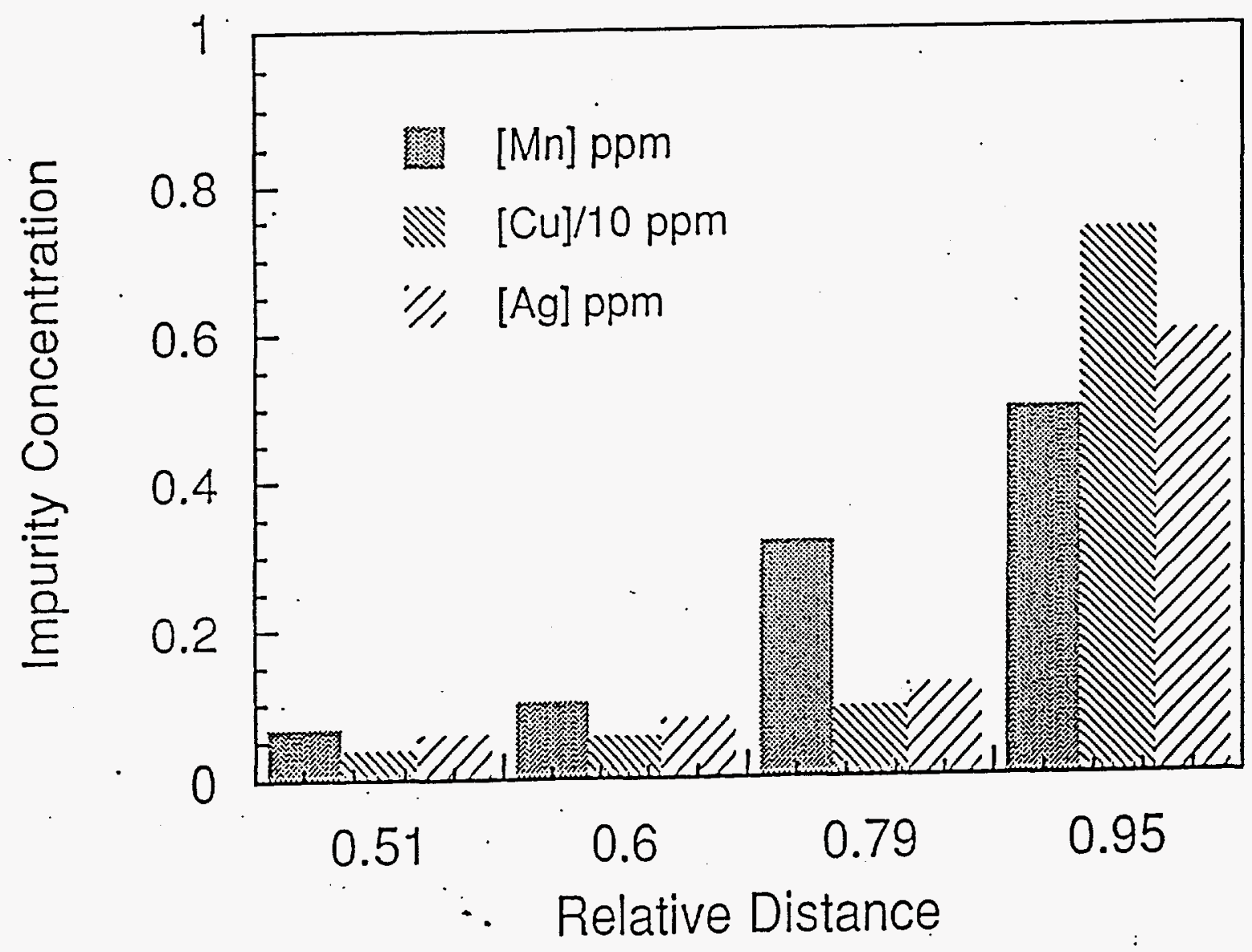


Figure 3. Experimentally measured concentration profile for $\mathrm{Mn}$ in zone refined lead iodide (circles) along with exponential fit to the data. This data yielded a segregation coefficient for $\mathrm{Mn}$ of 0.71 and in view of the good agreement between the model and the data this value is reasonably accurate.

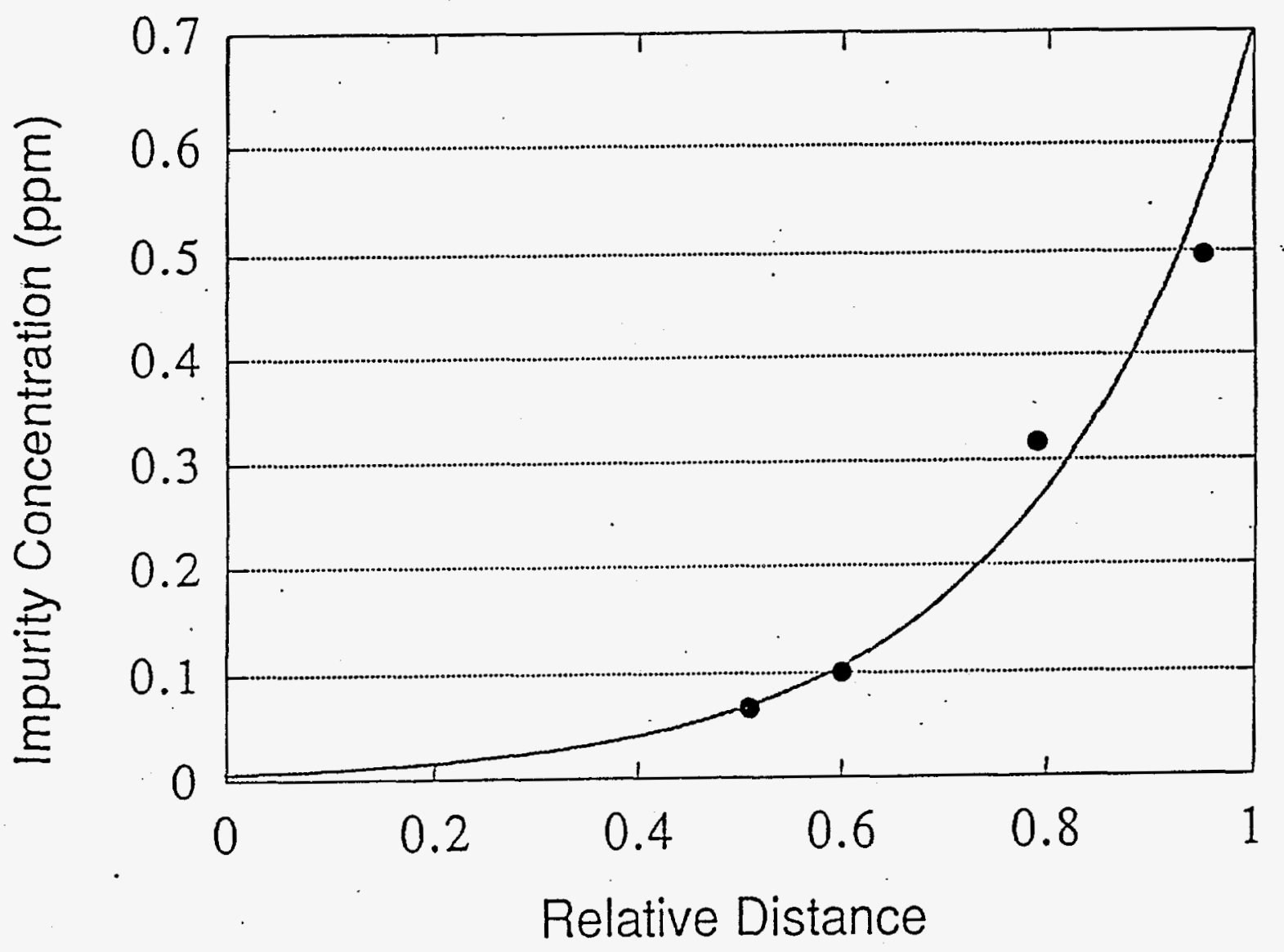


Figure 4. Low temperature $(4.2 \mathrm{~K})$ photoluminescence spectra of zone refined lead iodide samples obtained from the middle (upper spectrum) and the last to freeze (lower spectrum) region of an ingot. We observe more intense luminescence in the upper spectra (note that the lower spectra has been multiplied by a factor of three) indicating higher quality material in the middle of the ingot. Note also the relate intense deep luminescence and less well resolved near band edge luminescence in the last to freeze sample.

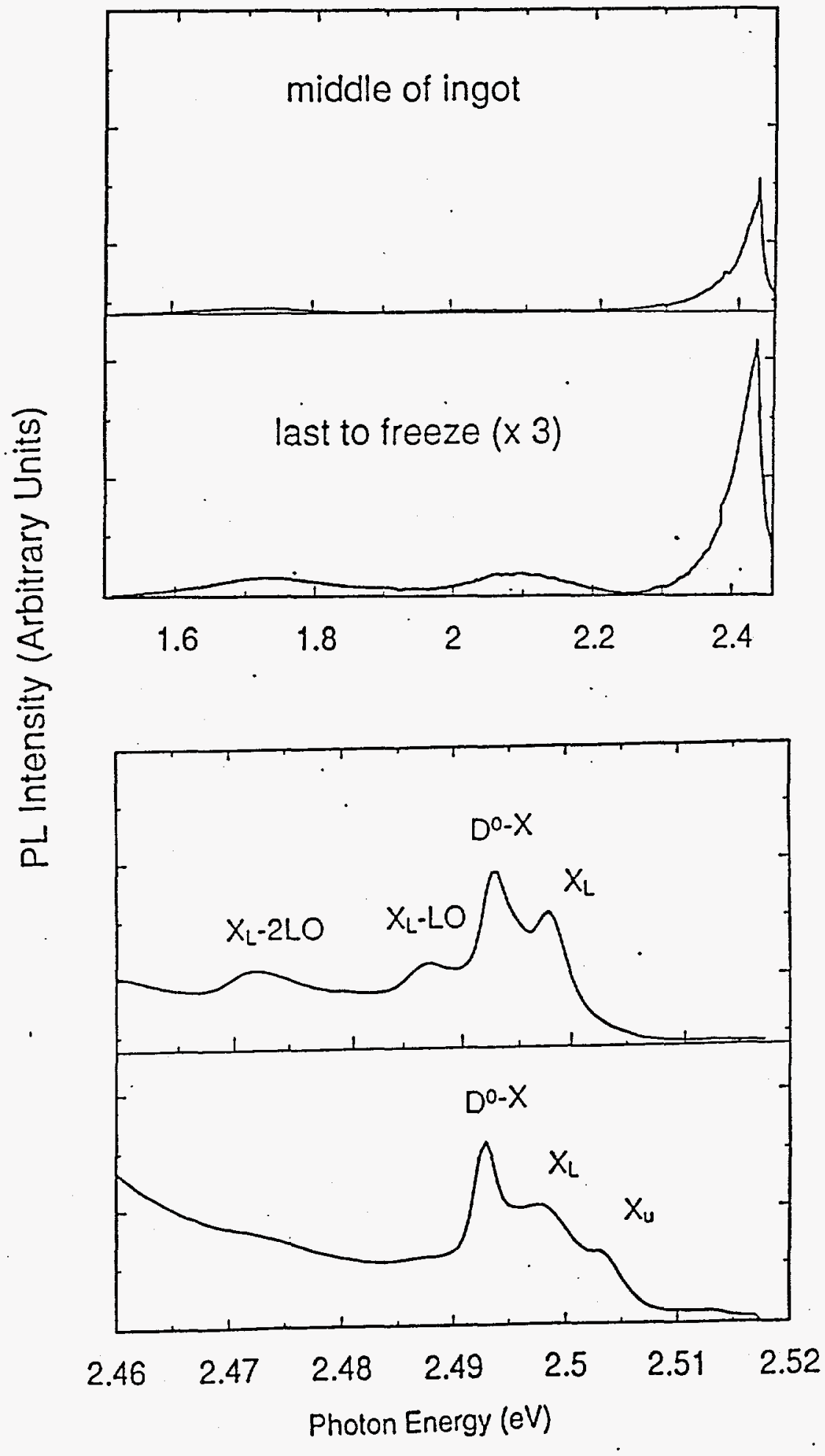




\title{
Phonon dispersion and polar-optical scattering in $2 \mathrm{H} \mathrm{PbI}_{2}$
}

\author{
Yia-Chung Chang \\ Department of Physics and Materials Research Laboratory \\ University of Illinois at Urbana-Champaign 1110 W. Green St., \\ Urbana, Ilinois 61801 \\ R. B. James \\ Advanced Electronics Manufacturing Technologies Department \\ Sandia National Lab, Livermore, CA 94550
}

(August 10, 1996)

\begin{abstract}
Theoretical studies of phonon dispersion curves and the polar-optical scattering process for $2 \mathrm{H} \mathrm{PbI}_{2}$ are presented. A rigid-ion model with anisotropic effective charges is used to calculate the phonon dispersion curves. The carrier mobility and its arisotropy due to polar-optical scattering as a function of temperature are calculated. Results are in good agreement with the experimental data.
\end{abstract}




\section{INTRODUCTION}

$\mathrm{PbI}_{2}$ is one of the candidate materials for room temperature $\mathrm{X}$-ray and $\gamma$-ray radiation detectors. [1] The electronic structures and optical properties 'were studied both experimentally[2-6] and theoretically.[7-9] It was determined that $\mathrm{PbI}_{2}$ is direct with both the conduction-band minimum and valence-band maximum occurring at the $\mathrm{A}$ point. The conduction band states are derived from the spin-orbit split $6 p$ orbitals of $\mathrm{Pb}$, while the valence-band states from the $6 s$ orbitals of $\mathrm{Pb}$ and $5 p_{z}$ orbitals of $\mathrm{I}$. The phonon properties have been studied via the neutron scattering [10], reflectivity [11,12], and Raman [13] measurements. The carrier mobilities were determined by Hall measurements [14] and I-V studies [15]. Up to now, theoretical calculations of the phonon modes and carrier transport properties have not been reported. The carrier transport is an important consideration for applications in room-temperature radiation detectors. In this paper, we present theoretical studies of both the phonon and transport properties of $\mathrm{PbI}_{2}$ via the use of a rigid-ion model and polar-optical scattering. Based on our previous studies of $\mathrm{HgI}_{2}$ [19], the polar-optical scattering is the dominant mechanism responsible for intrinsic carrier transport. Since $\mathrm{PbI}_{2}$ is similar to $\mathrm{HgI}_{2}$, we expect the same holds true. Our calculated electron mobilites based on polar-optical scattering alone are in fairly good agreement with experiment (within a factor of three), indicating that the currently observed electron mobilities are probably limited by the intrinsic scattering mechanism.

In section II, we present the rigid-ion model calculation for the phonon dispersion curves of $2 \mathrm{H} \mathrm{PbI}_{2}$. In section $\mathrm{III}$, we describe the polar-optical scattering for non-cubic crystals and examine the angular dependence of electron-phonon coupling constant for $\mathrm{PbI}_{2}$. In section IV, we discuss our calculated results for carrier scattering rates and mobilities as functions of temperature. A summary of the paper is given in section V.

\section{PHONON DISPERSION CURVES}

To calculate the phonon dispersion curves in the rigid-ion model, we need to fully utilize the symmetry properties of the crystal. The $2 \mathrm{H} \mathrm{PbI}_{2}$ has a hexagonal lattice with the unit cell depicted in FIG. 1. $\mathrm{Pb}$ (I) atoms are labeled by 1 (2 or 3 ) with or without superscript. The lattice constants used are $a=4.56 \dot{A}$ and $c=6.98 \dot{A}$. The primitive lattice vectors are $\mathbf{a}_{1}=(\sqrt{3} / 2,-1 / 2,0) a, \mathbf{a}_{2}=(0,1,0) a, \mathbf{a}_{3}=(0,0,1) c$ in Cartesian coordinates. The $\mathrm{Pb}$ atom in the unit cell is placed at the origin, and the two $\mathrm{I}$ atoms are placed at $\mathrm{u}_{1}=\frac{2}{3} \mathrm{a}_{1}+\frac{1}{3} \mathrm{a}_{2}+0.265 \mathrm{a}_{3}$ and $\frac{1}{3} \mathrm{u}_{2}=\mathrm{a}_{1}+\frac{2}{3} \mathrm{a}_{2}+0.265 \mathrm{a}_{3}$. The reciprocal lattice rectors are $b_{1}=(2 / \sqrt{3}, 0,0) \frac{2 \pi}{a}, b_{2}=(1 / \sqrt{3}, 1,0) \frac{2 \pi}{a}$, and $b_{3}=(0,0,1) \frac{2 \pi}{c}$. The first brillowin zone is shown in FIG. 3 of Ref. [8].

The point group of the crystal is $D_{3 d}$ with 12 elements. Six symmetry operations are denoted by $E, C_{3}, C_{3}^{-1}, \sigma_{d}, \sigma_{d} C_{3}, \sigma_{d} C_{3}^{-1}$, where $E$ is the identity, $C_{3}$ a three-fold rotation about the c-axis, and $\sigma_{d}$ an $x-z$ mirror plane. The matrix representation of the six operations are given in Table $\mathrm{I}$. The remaining six symmetry operations are products of the above with the inversion (denoted by $I$ ). The irreducible representations of the $D_{3 d}$ point group are denoted by $A_{1 g}, A_{2 u}, B_{g}, E_{g}$, and $E_{u}$. The $A B$ representations are one dimensional and the 
$E$ representations are two dimensional. The subscripts $g$ and $u$ denote symmetries which are even (gerade) and odd (ungerade) under inversion.

Since there are three atoms per unit cell, we expect to find nine phonon branches. The polarization vectors of zone-center phonons should transform according to the irreducible representations of the group $D_{3 d}$. Due to the existence of an inversion center which falls on $\mathrm{a} \mathrm{Pb}$ atom, the symmetry identification of the zone-center phonon modes is quite easy. First we classify the symmetry of phonon modes by its parity. A displacement of the $\mathrm{Pb}$ atom alone is denoted by a vector $d_{1}$, which has an odd parity, since the vector will flip sign under inversion. A simultaneous displacement of two I atoms in the unit cell in the same direction (denoted by $d_{+}=d_{2}+d_{3}$ ) also has an odd parity, while a simultaneous displacement of two $I$ atoms in opposite directions (denoted by $d_{-}=d_{2}-d_{3}$ ) has an even parity. Thus, odd-parity modes must have a polarization vector which is a linear combination of $d_{1}$ and $d_{+}$, and even-parity modes must behave like $d_{-}$. Taking into account the three possible directions $(x, y, z)$ we end up with six odd-parity modes (three acoustical and three optical) and three even-parity optical modes. The three $z$ vibrations transform according to the $A$ representation and the $x, y$ vibrations transform according the two-fold $E$ representation. Therefore, the three acoustical modes can be identified as the $A_{2 u}(L A)$ and $E_{u}(T A)$ modes (taking $z$ as the direction of propagation). The remaining six optical branches are identified as $A_{1 g}, E_{g}$ (even-parity) and $A_{2 u}, E_{u}$ (odd-parity) modes. The even-parity modes will be Raman active, while the odd-parity optical modes will be infrared active.

With the use of symmetry, the following relations among force-constant matrices are found. For the nearest-neighbor $\mathrm{Pb}-\mathrm{I}$ interactions, we have

$$
\Phi(1,2)=\left(\begin{array}{ccc}
A_{1} & 0 & D_{1} \\
0 & B_{1} & 0 \\
E_{1} & 0 & C_{1}
\end{array}\right)=C_{3} \Phi\left(1,2^{\prime}\right) C_{3}^{-1}=C_{3}^{-1} \Phi\left(1,2^{\prime \prime}\right) C_{3} .
$$

For the nearest-neighbor I-I interactions, we have

$$
\Phi(2,3)=\left(\begin{array}{lll}
A_{2} & D_{2} & E_{2} \\
D_{2} & B_{2} & F_{2} \\
E_{2} & F_{2} & C_{2}
\end{array}\right)=C_{3} \Phi\left(2,3^{\prime}\right) C_{3}^{-1}=C_{3}^{-1} \Phi\left(2,3^{\prime \prime}\right) C_{3} .
$$

For the next nearest-neighbor I-I interactions, we have

$$
\Phi(2, \overline{3})=\left(\begin{array}{lll}
A_{3} & D_{3} & E_{3} \\
D_{3} & B_{3} & F_{3} \\
E_{3} & F_{3} & C_{3}
\end{array}\right)=C_{3} \Phi\left(2, \overline{3}^{\prime}\right) C_{3}^{-1}=C_{3}^{-1} \Phi\left(2, \overline{3}^{\prime \prime}\right) C_{3} .
$$

Here $\overline{3}$ denotes an I atom 3 displaced by a lattice vector $-(0,0,1) c$ (see FIG. 1). All the other short-range couplings are ignored, as they are found to have less influence on the phonon dispersion curves.

The long-range Coulomb interaction matrix is calculated via the Ewald method by assuming a point charge $+e$ at the $\mathrm{Pb}$ sites and a point $-\mathrm{e} / 2$ at the I sites. To take into account the anisotropy, the $3 \times 3$ Coulomb matrix between any two ions is multiplied by the effective charge matrix given by 


$$
\left(\begin{array}{ccc}
e_{T}^{*} & 0 & 0 \\
0 & e_{T}^{*} & 0 \\
0 & 0 & e_{L}^{*}
\end{array}\right)
$$

where $e_{T}^{*}$ and $e_{L}^{*}$ are the transverse and longitudinal effective dynamic charges for the $\mathrm{Pb}$ atom, which are treated as adjustable parameters to fit the LO-TO splittings of the $E_{u}$ and $A_{2 u}$ optical modes. Including the 17 parameters for short-range force constants, we have a total of 19 adjustable parameters which are used in the fit of phonon dispersion curves.

We fit the lowest four phonon branches to the neutron scattering data of Ref. [10] and the zone center phonon frequericies for the $E_{u}(T O), E_{u}(L O), B_{g}, A_{1 u}(T O)$, and $A_{2 u}(L O)$ modes obtained from the far infrared reflectance [11,12] and Raman scattering data. [13] The best-fit parameters are given in Table II. The theoretical predictions for the phonon dispersion curves are shown in FIG. 2. The fit of the lowest four branches to the neutron scattering data is very good. The comparison of theory and experiment for the zone-center phonon frequencies is given in Table III and that for the sound velocities is given in Table IV. As seen in these tables, the agreement between theory and experiment is quite good.

Since $\mathrm{PbI}_{2}$ is anisotropic, we expect that the long-wavelength optical phonon modes to display an angular dispersion. This is shown in FIG. 3 , in which the phonon frequencies as functions of the angle $\theta$ (where $\cos \theta=q_{x} / q_{z}$ ) as the phonon wave vector approaches zero are plotted. It is noted that only the $E_{u}$ and $A_{2 u}$ modes which are infrared active have angular dispersion. The angular dispersion for the $E_{u}$ mode is quite large, and it tends to cross the dispersion curve of the $A_{2 u}$ mode. The two modes "anticross" at $\theta \approx 0.8 \frac{\pi}{2}$ as a result of level repulsion. Thus, there is a switch of characters at $\theta=\pi / 2$ such that the $E_{u}(L O)$ mode lies higher than the $A_{2 u}(T O)$ mode. As we shall see in the next two sections, this angular dependence plays a significant role in determining the anisotropy of the carrier mobilities.

\section{POLAR-OPTICAL SCATTERING}

In the $E_{u}$ and $A_{2 u}$ optical modes, ions with opposite charges vibrate against each other, thus giving rise to a long-range macroscopic electric field. The interaction of a carrier with such field is known as the polar optical scattering (or Frolich scattering). [21] Due to the strong polarity of the material, it is expected that the polar-optical scattering will be the dominant mechanism which determines the intrinsic carrier mobility at temperatures above about $77 \mathrm{~K}$. This has confirmed theoretically for $\mathrm{HgI}_{2}[19]$, a system of similar polarity. The electron-phonon interaction due to polar optical scattering associated with mode $j$ is given by

$$
H_{\text {el-ph }}=\frac{1}{\sqrt{N M_{\text {cell }}}} \sum_{q, \alpha} \sqrt{\frac{M_{\text {cell }}}{M_{\alpha}}} \frac{e}{v_{c}}\left[\frac{e_{\alpha, t}^{*} \hat{\epsilon}_{\alpha, l}^{(j)}}{\epsilon_{l}(\infty)}+\frac{e_{\alpha, t}^{*} \hat{\epsilon}_{\alpha, t}^{(j)}}{\epsilon_{t}(\infty)}\right] \cdot \hat{q}\left(\frac{4 \pi}{q}\right) Q_{\alpha j} e^{i q \cdot r}
$$

where $e$ is the free electron charge, $e_{\alpha, l}^{*}\left(e_{\alpha, t}^{*}\right)$ is the longitudinal(transverse) effective dynamic charge of ion $\alpha, \epsilon_{l}(\infty)\left[\epsilon_{t}(\infty)\right]$ is the longitudinal [transverse] high-frequency dielectric constant, $\hat{\epsilon}_{\alpha}^{(j)}$ is the polarization vector, and $v_{c}$ is volume of the unit cell. $e_{t}^{*}$ has been determined by reflectivity measurements to be $2.8 e$ for the $\mathrm{Pb}$ ion [11], while our theoretical value (obtained by fitting the LO-TO splitting of the $E_{u}$ mode) is 3.60 e (see Table II). $e_{i}^{i}$ has not been 
determined experimentally as it is difficult to obtain $\mathrm{PbI}_{2}$ crystals suitable for performing reflectivity measurements with the electric field of the incident photon parallel to the c-axis. The theoretical value obtained is $e_{l}^{*}=2.58$ which gives a resonable LO-TO splitting of the $A_{2 u}$ mode inferred from the dielectric measurement [16].

The effect of polar optical scattering can be described by an angular dependent coupling constant defined as

$$
C_{j}(\mathbf{q})=4 \pi\left|\sum_{\alpha} \sqrt{\frac{M_{c e l l}}{M_{\alpha}}} \frac{e}{v_{c}}\left[\frac{e_{\alpha, l}^{*} \hat{\epsilon}_{\alpha, l}^{(j)}}{\epsilon_{l}(\infty)}+\frac{e_{\alpha, t}^{*} \hat{\epsilon}_{\alpha, t}^{(j)}}{\epsilon_{t}(\infty)}\right] \cdot \hat{q}\right| .
$$

Note that $C_{j}(\theta) / q$ plays the same role as a deformation potential constant in the deformationpotential scattering.

Using the displacement vectors obtained by the rigid-ion model, we can obtain the angular dependent coefficients $C_{j}\left(\theta_{q}\right)$ for the $E_{u}$ and $A_{2 u}$ optical modes. In FIG. 4, we plot the angular dependent coupling coefficient $C_{j}$ for the two infra-red active optical branches $\left(E_{u}\right.$ and $\left.A_{2 u}\right)$. The two modes are found to couple strongly at finite values of $\theta$, resulting in an unusual angular dependence of $C_{j}$. In the uncoupled case (such as in $\left.\mathrm{HgI} \mathrm{I}_{2}\right), C_{j}(\theta)$ for the $E_{u}\left(A_{2 u}\right)$ mode should approximately behave like the function $\sin ^{2} \theta\left(\cos ^{2} \theta\right)$. However, with the strong coupling in the present case, the angular dependence is completely changed. At finite $\theta$, we can no longer assign the lower (upper) branch as the $E_{u}\left(A_{2 u}\right)$ mode. Thus, from now on, we shall simply call them the lower and upper branches.

The scattering rate from state $\mathbf{k}$ to $\mathbf{k}^{\prime}$ due to polar-optical scattering associated with branch $j$ is given by

$$
\begin{aligned}
W_{j}\left(\mathbf{k}, \mathbf{k}^{\prime}\right)= & \frac{1}{8 \pi^{2} \rho} \int \frac{C_{j}^{2}\left(\theta_{q}\right)}{\omega_{j}\left(\theta_{q}\right) q^{2}}\left\{n\left(\omega_{j}\left(\theta_{q}\right)\right) \delta_{\mathbf{k}^{\prime}, \mathbf{k}+q} \delta\left(E_{\mathbf{k}^{\prime}}-E_{\mathbf{k}}-\hbar \omega_{j}\left(\theta_{q}\right)\right)\right. \\
& \left.+\left[n\left(\omega_{j}\right)+1\right] \delta_{\mathbf{k}^{\prime}, \mathbf{k}-\mathrm{q}} \delta\left(E_{\mathbf{k}^{\prime}}-E_{\mathbf{k}}+\hbar \omega_{j}\right)\right\} d q
\end{aligned}
$$

For $k\left(k^{\prime}\right)$ along a general direction, the inclusion of both the anisotropy of band structure and the phonon dispersion is complicated. We therefor approximate $\omega_{j}\left(\theta_{q}\right)$ by a constant $\bar{\omega}_{j}$, which is taken to be the value of $\omega$ at the angle $\theta_{q}$ where the the coupling strength $C_{j}$ is the maximum. From FIG. 4 , we see that the maximum occurs at $\theta_{q}=0.75 \frac{\pi}{2}$ for the lower branch and at $\pi / 2$ for the upper branch. Thus, we have $\bar{\omega}_{1}=2.60 \mathrm{TH} z$ and $\bar{\omega}_{2}=3.396 \mathrm{THz}$ (see FIG. 3).

We define new variables

$$
q_{i}^{*}=\sqrt{\sigma} q_{i} \text { for } i=x, y
$$

and $q_{z}^{*}=q_{z}$, where $\sigma \equiv \frac{m_{i}^{*}}{m_{i}^{*}}$, and similarly for $\mathrm{k}^{*}\left(\mathrm{k}^{\prime *}\right)$, so that $E_{\mathrm{k}}\left(E_{\mathrm{k}^{\prime}}\right)$ becomes a spherical function of $\mathrm{k}^{*}\left(\mathrm{k}^{\prime *}\right)$. Eq. (3) becomes

$$
\begin{aligned}
& W_{j}\left(\mathrm{k}, \mathrm{k}^{\prime}\right)=\frac{\bar{C}_{j}^{2}}{8 \pi^{2} \rho \bar{\omega}_{j}} \int \frac{d \mathrm{q}^{*}}{q^{* 2}}\left\{n\left(\bar{\omega}_{j}\right) \delta_{\mathbf{k}^{-}, \mathbf{k}^{\bullet}+\mathbf{q}^{\cdot}} \delta\left(E_{\mathbf{k}^{\prime}}-E_{\mathrm{k}}-\hbar \bar{\omega}_{j}\right)\right. \\
& \left.+\left[n\left(\bar{\omega}_{j}\right)+1\right] \delta_{\mathbf{k}^{\cdot}, \mathrm{k}^{\cdot}-\mathbf{q}^{\cdot}} \delta\left(E_{\mathbf{k}^{\prime}}-E_{\mathrm{k}}+\hbar \bar{\omega}_{j}\right)\right\},
\end{aligned}
$$


where $\bar{C}_{j}^{2}$ is defined by

$$
\bar{C}_{j}^{2}=\int_{-1}^{1} \frac{C_{j}^{2}\left(\theta_{q}\right)}{\sin ^{2} \theta_{q}^{*}+\sigma \cos ^{2} \theta_{q}^{*}} d \cos \theta_{q}^{*} / 2 .
$$

The values of $\bar{C}_{j}$ are listed in TABLE V.

\section{CARRIER MOBILITY}

To calculate the carrier mobility, we also need the momentum relaxation time $\tau_{i}$ (the subscript $i$ denotes the direction of transport) which is related to the scattering rate via the expression [22]

$$
\frac{1}{\tau_{i}(E)}=\int k_{i}\left(k_{i}-k_{i}^{\prime}\right) W\left(\mathbf{k}^{\prime}, \mathbf{k}\right) d \mathbf{k}^{\prime} \delta\left(E-E_{\mathrm{k}}\right) d \mathrm{k} / \int k_{i}^{2} \delta\left(E-E_{\mathrm{k}}\right) d \mathrm{k},
$$

where $W\left(\mathrm{k}^{\prime}, \mathrm{k}\right)$ is the scattering rate given in the previous section. Substituting Eq.(5) into Eq. (6) we obtain

$$
\begin{gathered}
\frac{1}{\pi(E)}=\frac{3 \hbar^{3}}{16 \pi^{2} \rho\left(2 m_{l}^{*} E\right)^{3 / 2}} \sum_{j} \int_{-k_{m}}^{k_{m}} d k_{z} k_{z} \int d \phi_{q}^{*} \int_{0}^{\pi} d \theta_{q}^{*} \frac{C_{j}^{2}\left(\theta_{q}\right) \cos \theta_{q}^{*} \sin \theta_{q}^{*}}{\omega_{j}\left(\sin ^{2} \theta_{q}^{*}+\sigma \cos ^{2} \theta_{q}^{*}\right)} \\
\left\{-n\left(\omega_{j}\right)\left[q_{+}^{(1)} / R_{+}\left(q_{+}^{(1)}\right)+q_{+}^{(2)} / R_{+}\left(q_{+}^{(2)}\right)\right]+\left[n\left(\omega_{j}\right)+1\right]\left[q_{-}^{(1)} / R_{-}\left(q_{-}^{(1)}\right)+q_{-}^{(2)} / R_{-}\left(q_{-}^{(2)}\right)\right]\right\}, \\
\frac{1}{\tau_{t}(E)}=\frac{3 \hbar^{3}}{32 \pi^{2} \rho\left(2 m_{l}^{*} E\right)^{3 / 2}} \sum_{j} \int_{-k_{m}}^{k_{m}}\left(k_{m}^{2}-k_{z}^{2}\right)^{1 / 2} d k_{z} \int d \phi_{q}^{*} \int_{0}^{\pi} d \theta_{q}^{*} \frac{C_{j}^{2}\left(\theta_{q}\right) \sin ^{2} \theta_{q}^{*} \cos \phi_{q}^{*}}{\omega_{j}\left(\sin ^{2} \theta_{q}^{*}+\sigma \cos ^{2} \theta_{q}^{*}\right)} \\
\left\{-n\left(\omega_{j}\right)\left[q_{+}^{(1)} / R_{+}\left(q_{+}^{(1)}\right)+q_{+}^{(2)} / R_{+}\left(q_{+}^{(2)}\right)\right]+\left[n\left(\omega_{j}\right)+1\right]\left[q_{-}^{(1)} / R_{-}\left(q_{-}^{(1)}\right)+q_{-}^{(2)} / R_{-}\left(q_{-}^{(2)}\right)\right]\right\},
\end{gathered}
$$

where $k_{m}=\sqrt{2 m_{l}^{*} E} / \hbar, R_{ \pm}(q)=\frac{\hbar^{2}}{m_{i}^{*}}\left|q \pm\left(k_{z} \cos \theta_{q}^{*}+\left(k_{m}^{2}-k_{z}^{2}\right)^{1 / 2} \sin \theta_{q}^{*} \cos \phi_{q}^{*}\right)\right| \cdot q_{ \pm}^{(1)}$ and $q_{ \pm}^{(2)}$ are two positive real roots of the equation

$$
\frac{\hbar^{2}}{2 m_{l}^{*}}\left[q^{2} \pm 2 q\left(k_{z} \cos \theta_{q}^{*}+\left(k_{m}^{2}-k_{z}^{2}\right)^{1 / 2} \sin \theta_{q}^{*} \cos \phi_{q}^{*}\right)\right]= \pm \hbar \omega_{j} .
$$

Here $\theta_{q}^{*}$ is the polar angle of $\mathbf{q}^{*}$ with respect to the $c$ axis and $\phi_{q}^{*}$ is the azimuthal angle between $\mathrm{q}^{*}$ and $\mathbf{k}$.

If we replace $\frac{C_{j}^{2}\left(\theta_{q}\right)}{\sin ^{2} \theta_{q}^{*}+\sigma \cos ^{2} \theta_{q}}$ by its spherically averaged values, $\bar{C}_{j}$ and $\omega_{j}\left(\theta_{q}\right)$ by $\bar{\omega}_{j}$ [values evaluated at maximum $C_{j}\left(\theta_{q}\right)$, the above results reduce to [22]

$$
\frac{i}{\tau(E)}=\frac{\bar{C}_{j}^{2}}{4 \pi \rho \hbar \bar{\omega}_{j} v_{E}} n\left(\bar{\omega}_{j}\right)\left[\sqrt{1+\frac{\hbar \bar{\omega}_{j}}{E}}-\frac{\hbar \bar{\omega}_{j}}{E} \sinh ^{-1} \sqrt{\frac{E}{\hbar \bar{\omega}_{j}}}\right]
$$




$$
+\left[n\left(\bar{\omega}_{j}\right)+1\right]\left[\sqrt{1-\frac{\hbar \bar{\omega}_{j}}{E}}+\frac{\hbar \bar{\omega}_{j}}{E} \sinh ^{-1} \sqrt{\frac{E}{\hbar \bar{\omega}_{j}}-1}\right]
$$

where $v_{E}=\sqrt{2 E / m_{l}^{*}}$.

Momentum relaxation rates $\left(\frac{1}{\eta}\right.$ and $\left.\frac{1}{\tau_{t}}\right)$ at $300 \mathrm{~K}$ due to polar-optical scattering are plotted in FIG. 5 for three different mass anisotropies $\frac{m_{i}}{m_{i}^{*}} \equiv \sigma=1,2$, and 5 with $m_{i}^{*}$ assumed equal to the free electron mass, $m_{0}$. The solid (dashed) curves are for $\frac{1}{\eta}\left(\frac{1}{\tau_{1}}\right)$ and the dotted curves are the polar-optical scattering rates $\left(\frac{1}{\tau}\right)$ calculated in the spherical model according to Eq. (9). For carriers with a longitudinal effective mass differing from $m_{0}$, the result simply scales according to $\sqrt{m_{i}^{*} / m_{0}}$ [see Eq. (9)]. In FIG. 5, the two shoulder structures are due to the onset of the optical-phonon emission for the two branches with the higher branch being stronger. It is noted that even for spherical band $(\sigma=1) \frac{1}{\eta}$ and $\frac{1}{\tau_{t}}$ are different by about $30 \%$ due to the anisotropy in the scattering rate.

Finally the mobilities are related to the momentum relaxation times via [22]

$$
\begin{aligned}
& \mu_{l}=\frac{e}{m_{l}^{*}} \frac{\left\langle E \tau_{l}(E)\right\rangle}{\langle E\rangle}, \\
& \mu_{t}=\frac{e}{m_{t}^{*}} \frac{\left\langle E \tau_{t}(E)\right\rangle}{\langle E\rangle},
\end{aligned}
$$

where \{\rangle denotes a thermal average with a Boltzmann distribution in the non-degenerate limit. To simplify the calculation, we use the spherical-model expression [Eq. (9)] for the polar-optical scattering, but scale it by appropriate factors to take into account the anisotropy of scattering rates. As shown in FIG. $5, \tau_{l}^{-1}$ and $\tau_{t}^{-1}$ differ from the spherical results $(I / \tau)$ approximately by an energy-independent factor. We find $\tau_{l}^{-1} \approx f_{l} \tau^{-1}$ and $\tau_{t}^{-1} \approx f_{t} \tau^{-1}$ with $f_{t}=1.0,0.812,0.701$ and $f_{t}=1.287,1.283,1.439$ for $\sigma=1,2,5$.

FIG. 6 shows the mobility as a function of temperature for a carrier with $m_{l}^{*}=m_{0}$ and with three different values of $\frac{m_{i}^{*}}{m_{i}^{*}}$. Note that the carrier mobility scales like $m_{l}^{*-3 / 2}$ (since $\tau$ scales like $\left.m_{l}^{*-1 / 2}\right)$. The existing estimates of the effective masses of $\mathrm{PbI}_{2}$ are quite uncertain. The cyclotron measurement [14] gives an electron polaron mass $\left(m_{l}^{*} m_{t}^{*}\right)^{\frac{1}{2}}=0.68 \pm 0.16 m_{0}$. Based on the band structure calculation [9], $m_{l}^{*}$ is greater than $m_{t}^{*}$, but the ratio is not known accurately. Assuming $\sigma=2$, we obtain $m_{l}^{*} \approx 0.96 m_{0}$ and the electron mobilities $\mu_{l}\left(\mu_{t}\right) \approx 110(140) \mathrm{cm}^{2} / V s$ and $20(25) \mathrm{cm}^{2} / V s$ at $100 \mathrm{~K}$ and $300 \mathrm{~K}$, respectively. The results are in agreement with the experimental data within a factor of 3. [14,15] The temperature dependence of the mobility goes approximately like $1 / T^{1.3}$ for temperatures between $40 \mathrm{~K}$ and $100 \mathrm{~K}$, which agrees fairly well with the measurements of Ref. [14]. For holes, the calculated hole effective masses are $m_{l}^{*} \approx 1.67 m_{0}$ and $m_{t}^{*} \approx 0.35 m_{0}$ which gives $m_{l}^{*} / m_{i}^{*} \approx 5$. [23] Using these values, we obtain the room-temperature hole mobilities $\mu_{t} \approx 34 \mathrm{~cm}^{2} / V s$ and $\mu_{\mathrm{l}} \approx 15 \mathrm{~cm}^{2} / V s$. These numbers are about one order of magnitude higher than the observed hole mobilities [15], indicating that the extrinsic scattering mechanism may be important here.

Although there are uncertainties with the effective dynamic charges and the effective masses used in the calculation, the over-all estimate should be correct within a factor of 
three. The fairly good agreement for the electron mobilities between theory and experiment at room temperature (within a factor of three) indicates that the intrinsic mechanism due to polar-optical scattering is probably the dominant factor in explaining the observed electron mobility, while the hole mobility may be more influenced by the extrinsic scattering mechanisms.

\section{CONCLUSION}

We have presented detailed.theoretical studies of the phonon dispersion curves, electronphonon interaction due to polar-optical scattering, and carrier mobilities of $2 \mathrm{H}_{\mathrm{HbI}}$. The phonon dispersion curves obtained in the rigid-ion model with 19 empirical parameters agree well with the neutron scattering data for the lowest four branches and with the far-infrared as well as Raman scattering data for all zone-center optical modes. The amount of angular dispersion for the two infrared active optical modes $\left(E_{u}\right.$ and $\left.A_{2 u}\right)$ is also in agreement with the reflectivity measurements. The effective dynamic charges which are adjusted to give rise to the correct angular dispersion for the $E_{u}$ and $A_{2 u}$ optical modes are used in the calculation of the polar-optical scattering. The carrier mobilities due to the polar-optical scattering are then calculated. Both the magnitudes and anisotropy of carrier mobilities and their temperature dependence from 40 to $100 \mathrm{~K}$ are found in qualitative agreement with experiment.

\section{ACKNOWLEDGMENTS}

This work is supported in part by the Department of Energy. 
TABLE I. The matrix representation of symmetry operations of the point group for $\mathrm{PbI}_{2}$.

$$
\begin{array}{lc}
E=\left(\begin{array}{lll}
1 & 0 & 0 \\
0 & 1 & 0 \\
0 & 0 & 1
\end{array}\right) & \sigma_{d}=\left(\begin{array}{ccc}
1 & 0 & 0 \\
0 & -1 & 0 \\
0 & 0 & 1
\end{array}\right) \\
C_{3}=\left(\begin{array}{ccc}
-\frac{1}{2} & \frac{\sqrt{3}}{2} & 0 \\
-\frac{\sqrt{3}}{2} & -\frac{1}{2} & 0 \\
0 & 0 & 1
\end{array}\right) & C_{3}^{-1}=\left(\begin{array}{ccc}
-\frac{1}{2} & -\frac{\sqrt{3}}{2} & 0 \\
\frac{\sqrt{3}}{2} & -\frac{1}{2} & 0 \\
0 & 0 & 1
\end{array}\right) \\
C_{3} \sigma_{d}=\left(\begin{array}{ccc}
-\frac{1}{2} & -\frac{\sqrt{3}}{2} & 0 \\
\frac{\sqrt{3}}{2} & \frac{1}{2} & 0 \\
0 & 0 & 1
\end{array}\right) C_{3}^{-1} \sigma_{d}=\left(\begin{array}{ccc}
-\frac{1}{2} & \frac{\sqrt{3}}{2} & 0 \\
-\frac{\sqrt{3}}{2} & \frac{1}{2} & 0 \\
0 & 0 & 1
\end{array}\right) \\
\hline
\end{array}
$$

TABLE II. Parameters used in the rigid-ion model (measured in units of $e^{2} / v_{c}$, where $v_{c}$ is the unit-cell volume). $e_{l}^{*}$ and $e_{t}^{*}$ are dimensionless.

\begin{tabular}{lcccccc}
\hline \hline$A_{1}$ & $B_{1}$ & $C_{1}$ & $D_{1}$ & $E_{1}$ & $e_{l}^{*}$ & $e_{t}^{*}$ \\
-9.18828 & 2.93684 & -6.38286 & 3.82050 & -10.09855 & 3.59933 & 2.57632 \\
$A_{2}$ & $B_{2}$ & $C_{2}$ & $D_{2}$ & $E_{2}$ & $F_{2}$ & \\
-3.30617 & 2.71029 & -6.01193 & 0.94633 & 3.60113 & -2.87162 \\
$A_{3}$ & $B_{3}$ & $C_{3}$ & $D_{3}$ & $E_{3}$ & $F_{3}$ & \\
-1.35993 & -4.65746 & 3.27137 & -0.94474 & -1.86858 & 4.62527 & \\
\hline
\end{tabular}


TABLE III. Zone-center phonon frequencies (in $\mathrm{THz}$ ) for $\mathrm{PbI}_{2}$ obtained in the present rigidion model and from various experimental measurements.

\begin{tabular}{lcccccc}
\hline \hline & $E_{u}(T O)$ & $E_{u}(L O)$ & $E_{g}$ & $A_{1 g}$ & $A_{2 u}(T O)$ & $A_{2 u}(L O)$ \\
\hline Theory & 1.578 & 3.004 & 2.382 & 2.934 & 2.861 & 3.396 \\
Experiment & $1.55^{a}$ & $3.18^{\mathrm{b}}$ & $2.34^{\mathrm{b}}$ & $2.94^{\mathrm{b}}$ & $2.85^{\mathrm{b}}$ & $3.39^{\mathrm{b}}$ \\
\hline \hline
\end{tabular}

a. Lucovsky et al. (Ref. [11]).

b. Grisel and Schmid (Ref. [13]).

TABLE IV. Sound velocities (in $10^{5} \mathrm{~cm} / \mathrm{s}$ ) of acoustical branches for $\mathrm{PbI}_{2}$ obtained in the present rigid-ion model. The values in parentheses are experimental data taken from Ref. [10].

\begin{tabular}{lcc}
\hline \hline$[00 \xi]$ & {$[\xi \xi 0]$} & $\cdot[\xi 00]$ \\
\hline$v_{l}=1.392(1.54)$ & $v_{l}=1.892(1.81)$ & $v_{l}=1.944(1.86)$ \\
$v_{t}=1.073(0.93)$ & $v_{t, \|}=1.438(1.10)$ & $v_{t, \|}=1.225(1.07)$ \\
& $v_{t, \perp}=0.777(0.86)$ & $v_{t, \perp}=0.990(0.94)$ \\
\hline \hline
\end{tabular}

TABLE V. Spherical average of the coupling coefficients for polar-optical scatterings $\left(\bar{C}_{j .}\right)$ associated with $E_{u}$ and $A_{2 u}$ modes for three different longitudinal to transverse effective mass ratios $(\sigma)$. The units are $\mathrm{eV} / \dot{A}^{2}$.

\begin{tabular}{llll}
\hline \hline & $\sigma=1$ & $\sigma=2$ & $\sigma=5$ \\
\hline$E_{\vartheta}$ & 0.737 & 0.671 & 0.574 \\
$A_{2}$ & 1.378 & 1.275 & 1.134 \\
\hline \hline
\end{tabular}




\section{REFERENCES}

[1] see for example, J. C. Lund, F. Olschner, and A. Burger in Semiconductors and Semimetals, edited by T. E. Schlesinger and R. B. James, Academic Press (1995), Ch. 11, and references therein.

[2] G. Margaritondo, J. E. Rowe, M. Schlüter, and G. K. Wertheim, Phys. Rev. B 16, 2934 (1977).

[3] I. Imai, J. Phys. Chem. Solids 22, 81 (1961).

[4] G. Baldini and S. Franchi, Phys. Rev. Lett. 26, 503 (1971).

[5] G. Harbeke and E. Tosatti, Phys. Rev. Lett. 28, 1567 (1972).

[6] I. Baltog, I. Piticu, M. Constantinescu, C. Ghita, and L. Ghita, Phys. Stat. Sol. (a)52, 103 (1979).

[7] E. Doni, G. Grosso, and G. Spavieri, Solid State Common. 11, 493 (1972).

[8] L. Ch. Schlüter and M. Schlüter, Phys. Stat. Sol. (b) 57, 145 (1973).

[9] L. Ch. Schlüter and M. Schlüter, Phys. Rev. B9, 1652 (1974).

[10] B. Dorner, R. E. Ghosh, and G. Harbeke, Phys. Stat. Sol. (b) 73, 655 (1976).

[11] G. Lucovsky, R. M. White, W. Y. Liang, R. Zallen, and Ph. Scmid, Solid St. Commun. 18, 811 (1978).

[12] J. Hiraishi, K. Tani, and T. Tamura, J. Chem. Phys. 71, 554 (1979).

[13] A. Grisel and Ph. Schmid, Phys. Stat. Sol. (b) 73, 587 (1976).

[14] P. D. Bloch, J. W. Hodby, T. E. jenkins, D. W. Stacey, G. Lang, F. Lery, and C. Schwab, J. Phys. C: Solid St. Phys. 11, 4997 (1978).

[15] R. Minder, G. Ottaviani, and C. Canali, J. Phys. Chem. Solids 37, 417 (1976).

[16] A. E. Dugan, H. K. Henisch, J. Phys. Chem. Solids 35, 1269 (1974).

[17] I. Baltog, I. Piticu, M. Gonstantinescu, C. Ghita, and L. Ghita, Phys. Stat. Sol. (b) 52, $103(1979)$.

[18] K. Kunc, Ann. Phys. (Paris) 8, 319 (1973).

[19] Y. C. Chang and R. B. James, Phys. Rev. B (submitted).

[20] H. K. Sim, Y. C. Chang and R. B. James, Phys. Rev. B 49, 4559 (1994).

[21] H. Frölich, Proc. Roy. Soc. A 160, 230 (1937).

[22] B. K. Ridley, Quantum Process in Semiconductors.

[23] G. Grosso, unpublished (quoted in Ref. [14]). 


\section{FIGURE CAPTIONS}

FIG. 1. (a) Crystal structure of $\mathrm{PbI}_{2}$ with $\mathrm{Pb}$ atom labeled by 1 and $\mathrm{I}$ atoms labeled by 2 and 3. (b) Projection of the crystal onto the xy-plane. The nearest-neighbor I atoms centered at $\mathrm{Pb}$ atom 1 are labelled by 2, 2', and 2"in counter-clockwise order. Similarly, the nearest-neighbor I atoms centered at I atom 2 are labelled by 3,3 ', and $3^{\prime \prime}$.

FIG. 2. Phonon dispersion curves of $\mathrm{PbI}_{2}$ obtained by the present rigid-ion model. The symmetries of zone-center optical modes are marked.

FIG. 3. Angular dispersion of phonon frequencies for $\mathrm{PbI}_{2}$ deduced from the present rigidion model.

FIG. 4. Angular dependence of the coupling coeficient for polar optical phonon scattering for the two infra-red active modes.

FIG. 5. Momentum relaxation rates associated with polar-optical scattering at $300 \mathrm{~K}$ as functions of the carrier energy for three values of mass anisotropies: $(a) \sigma=1,(b) \sigma=2$, (c) $\sigma=5$. Solid curves: $\tau_{l}^{-1}$. Dashed curves: $\tau_{t}^{-1}$. Dotted curve: $\tau^{-1}$ (spherical model). FIG. 6. Carrier mobilities as functions of temperature for three values of mass anisotropies, $\sigma=1,2$, and 5 . Solid (dotted) curves are for transport parallel (perpendicular) to the $c$ axis. 
(a)

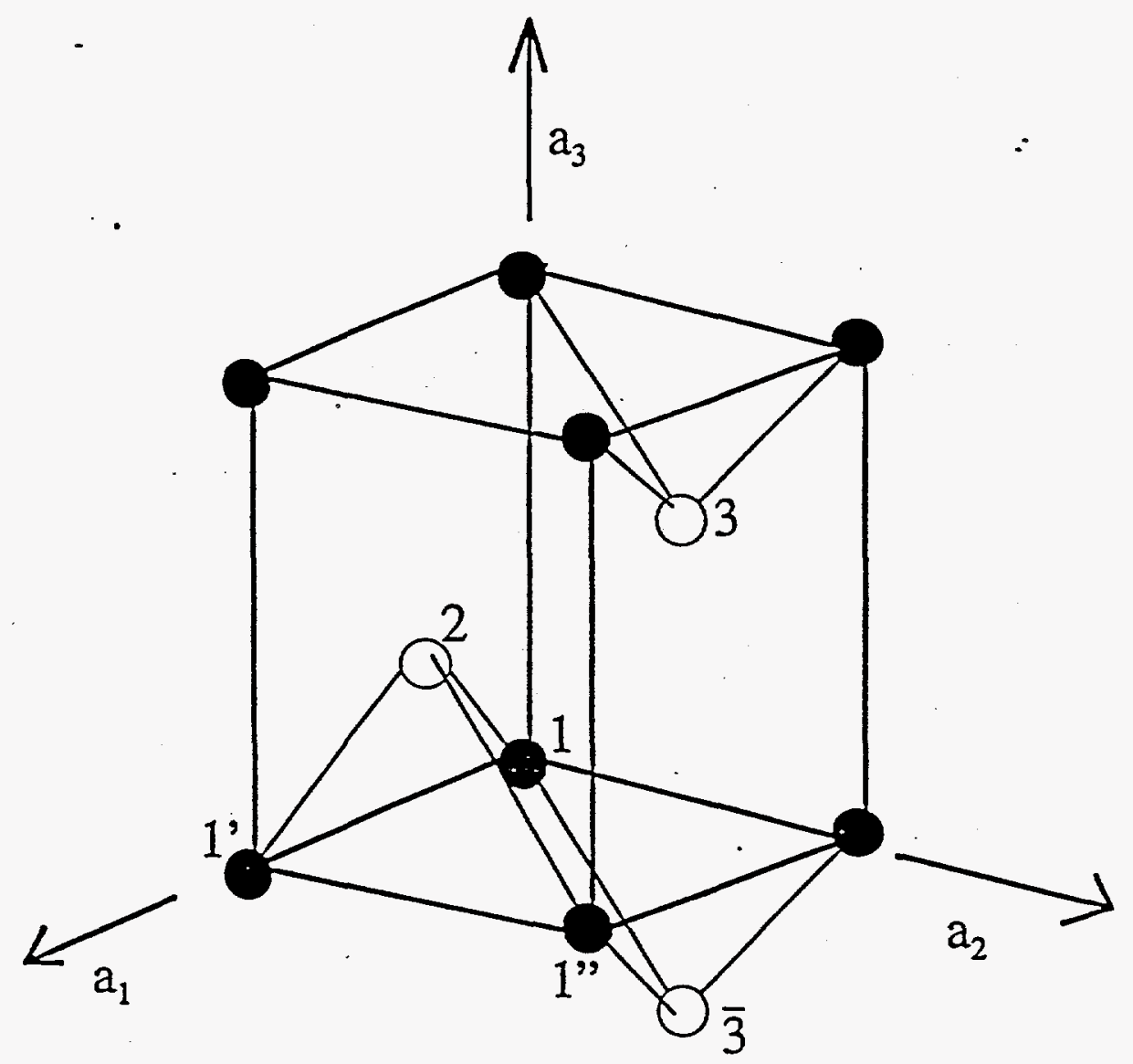

4

(b)

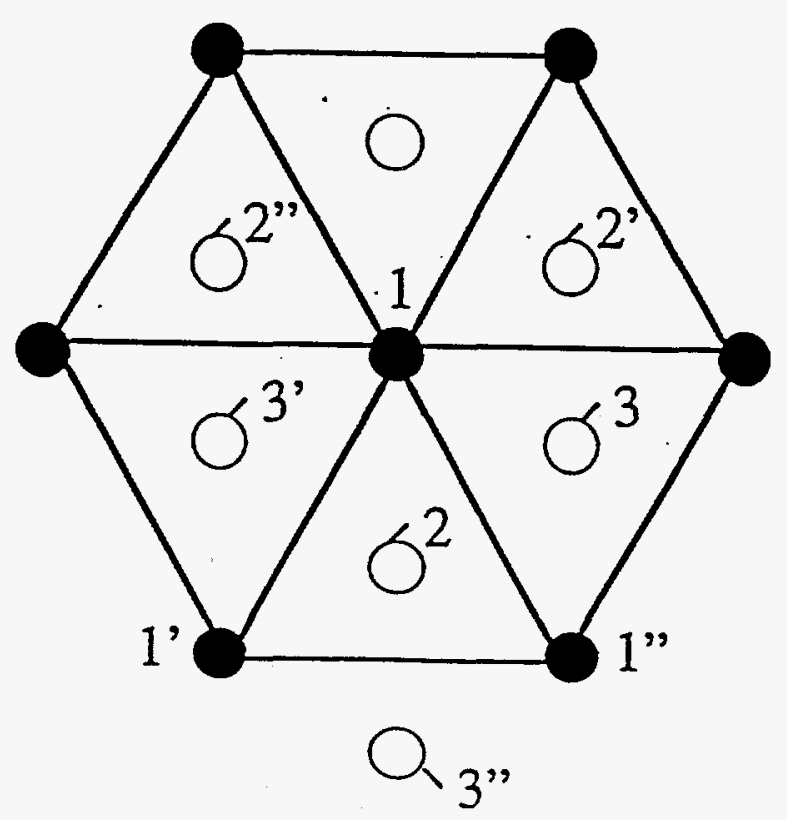

Fig. 1 


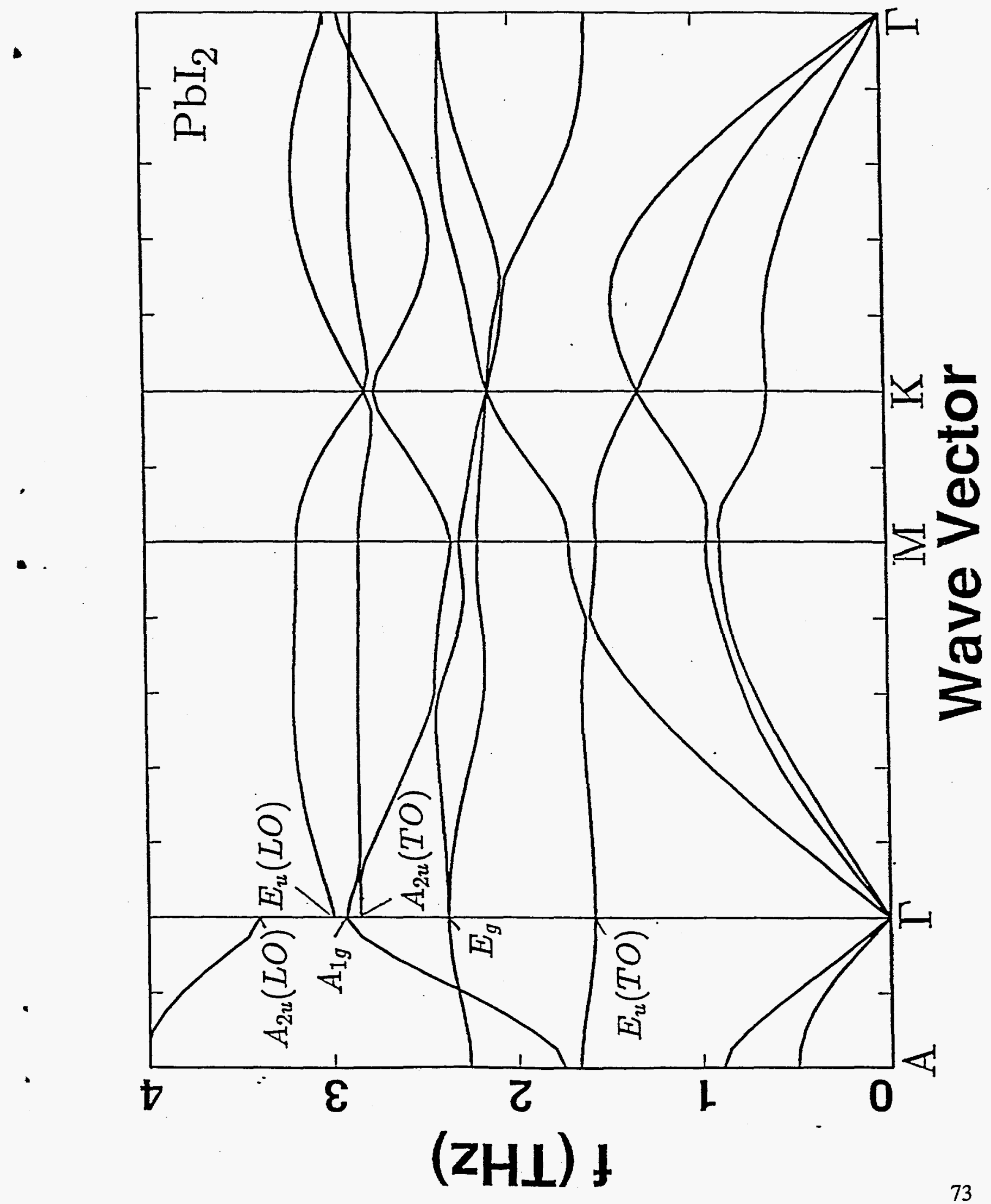




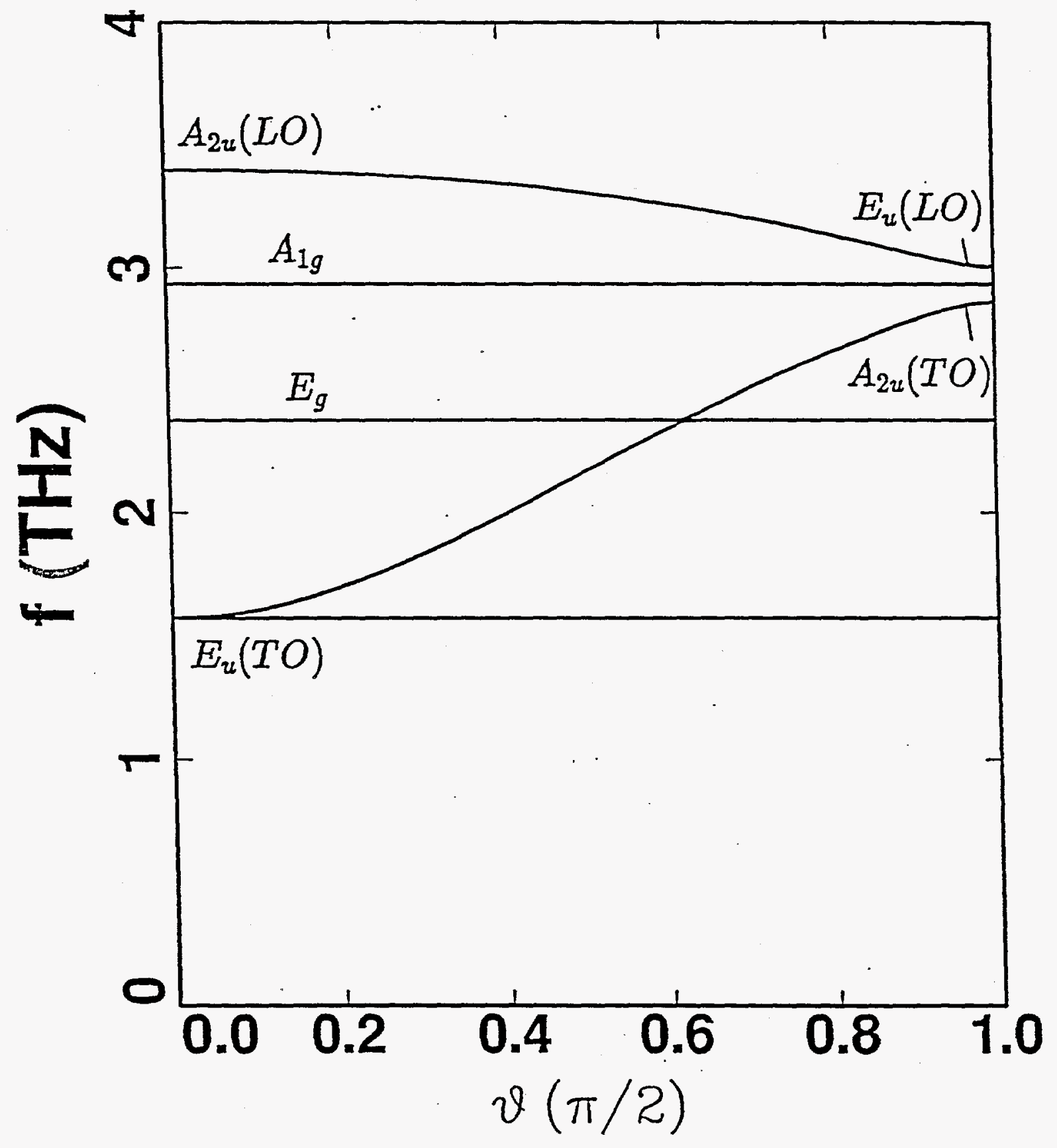




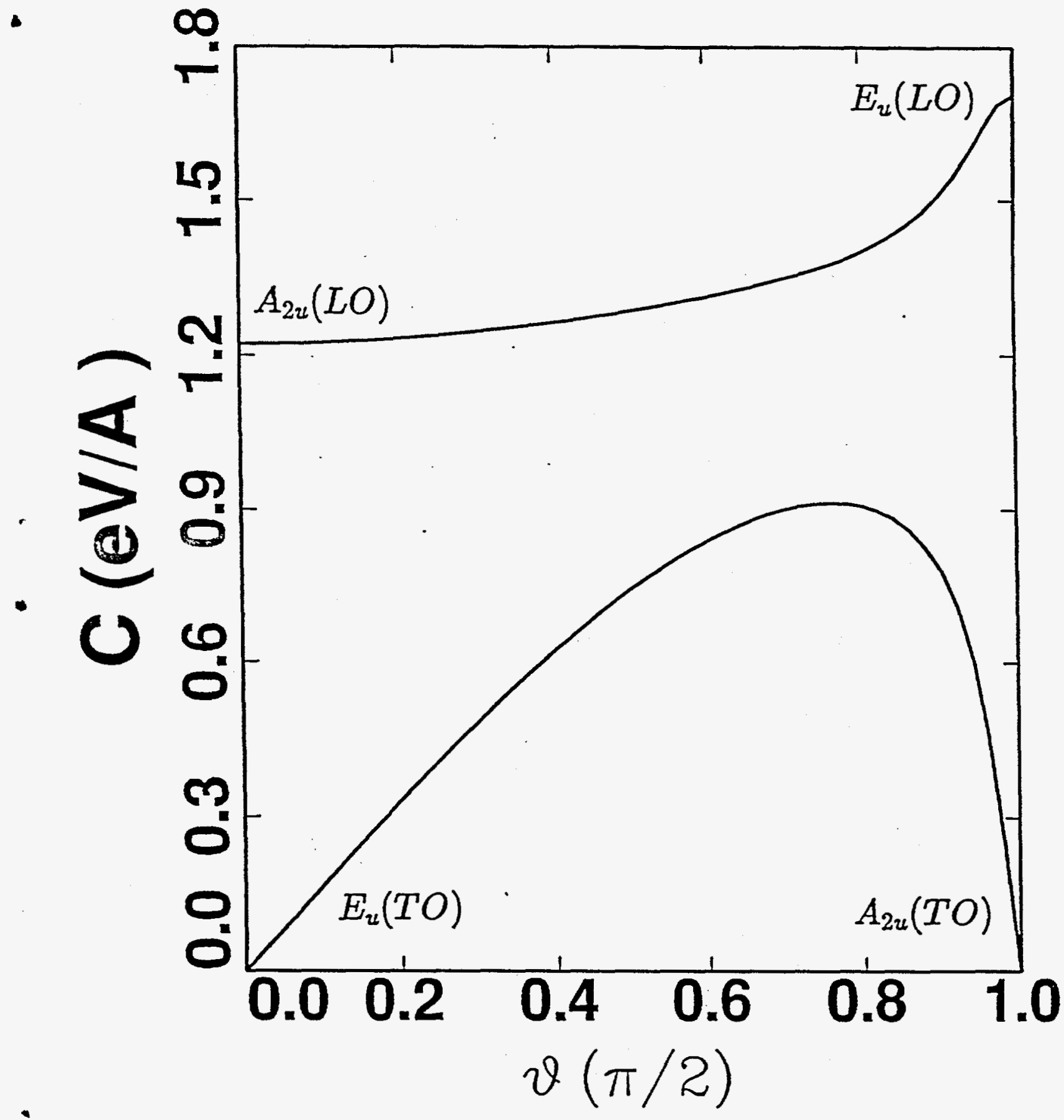




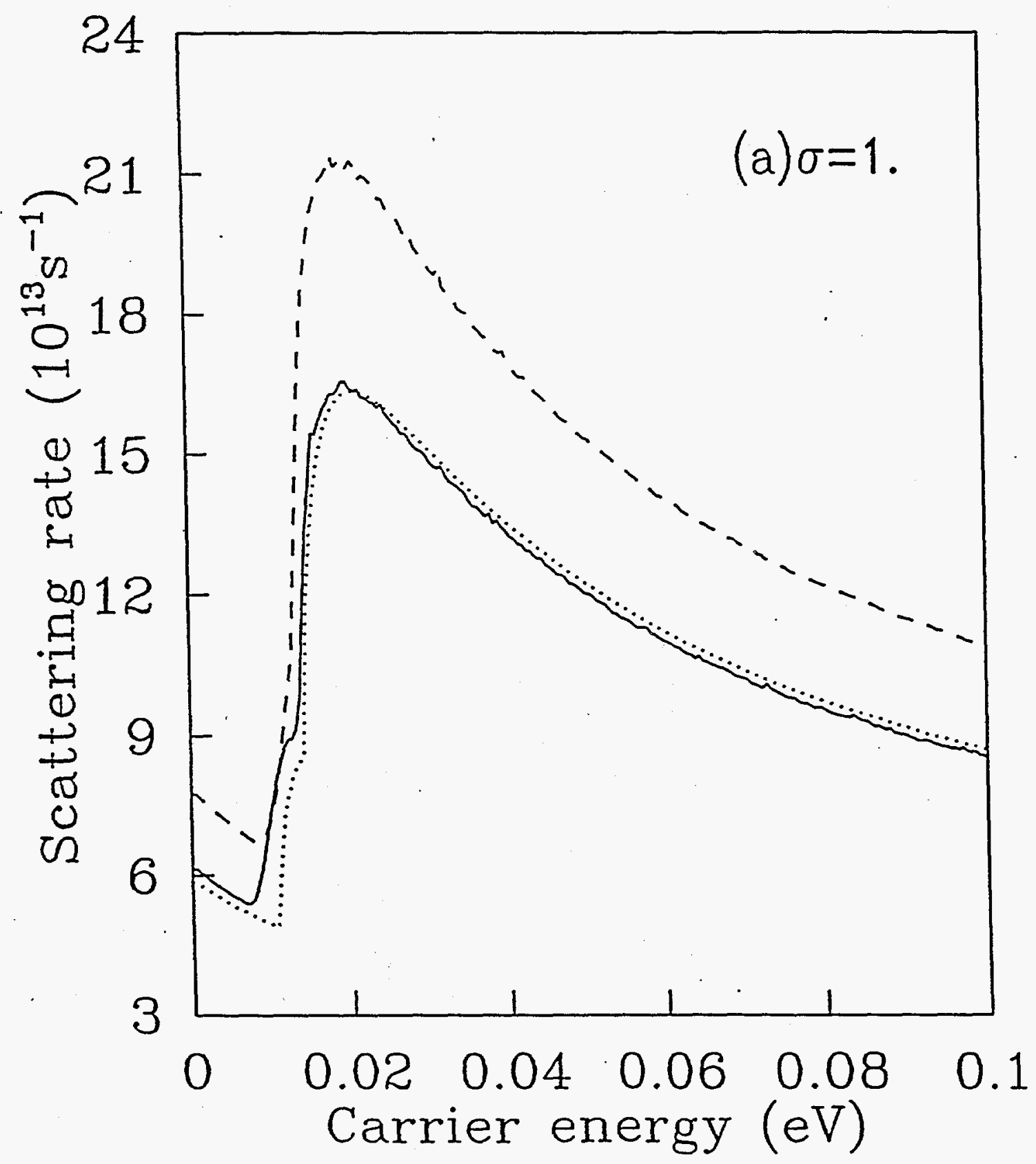




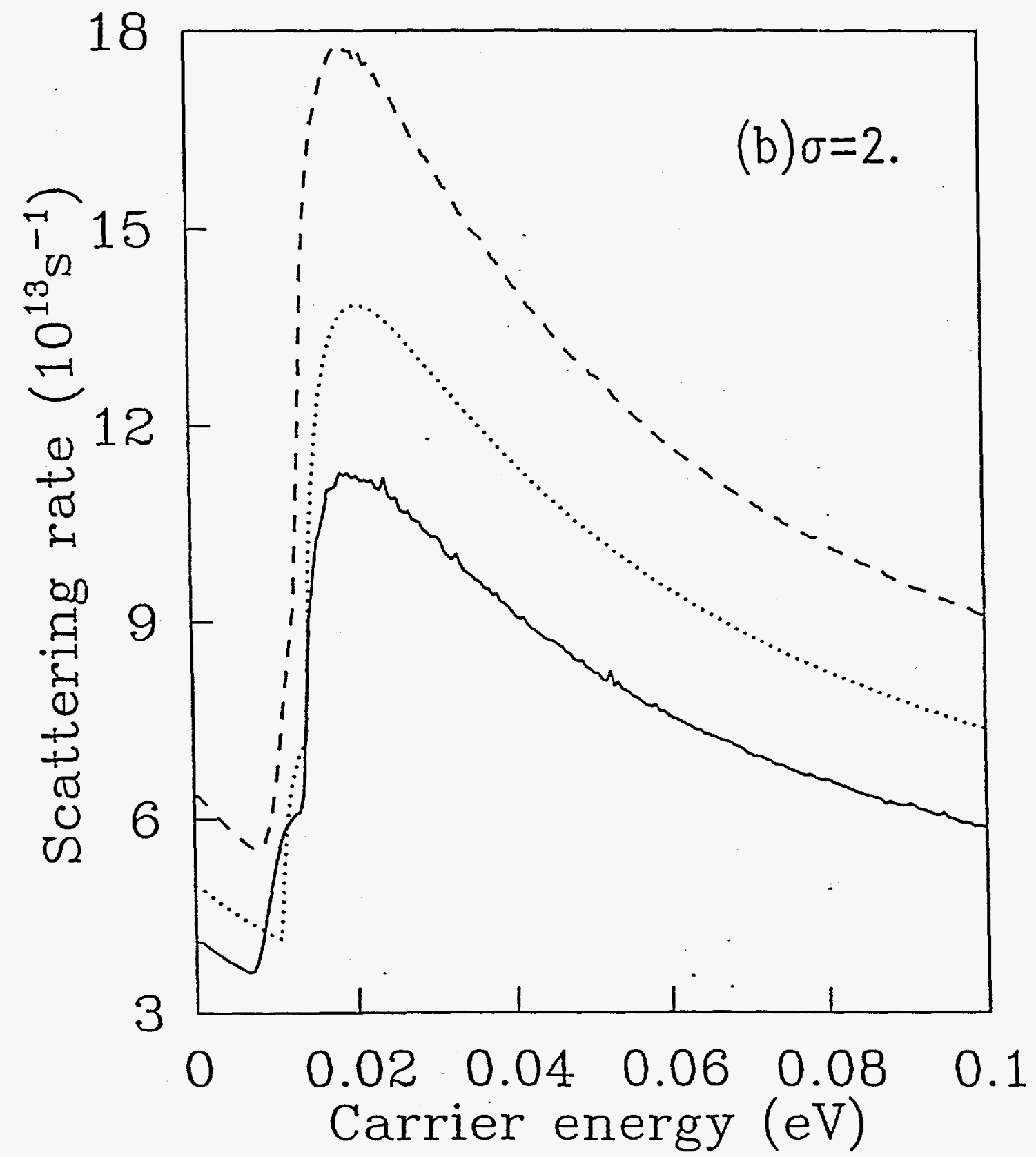




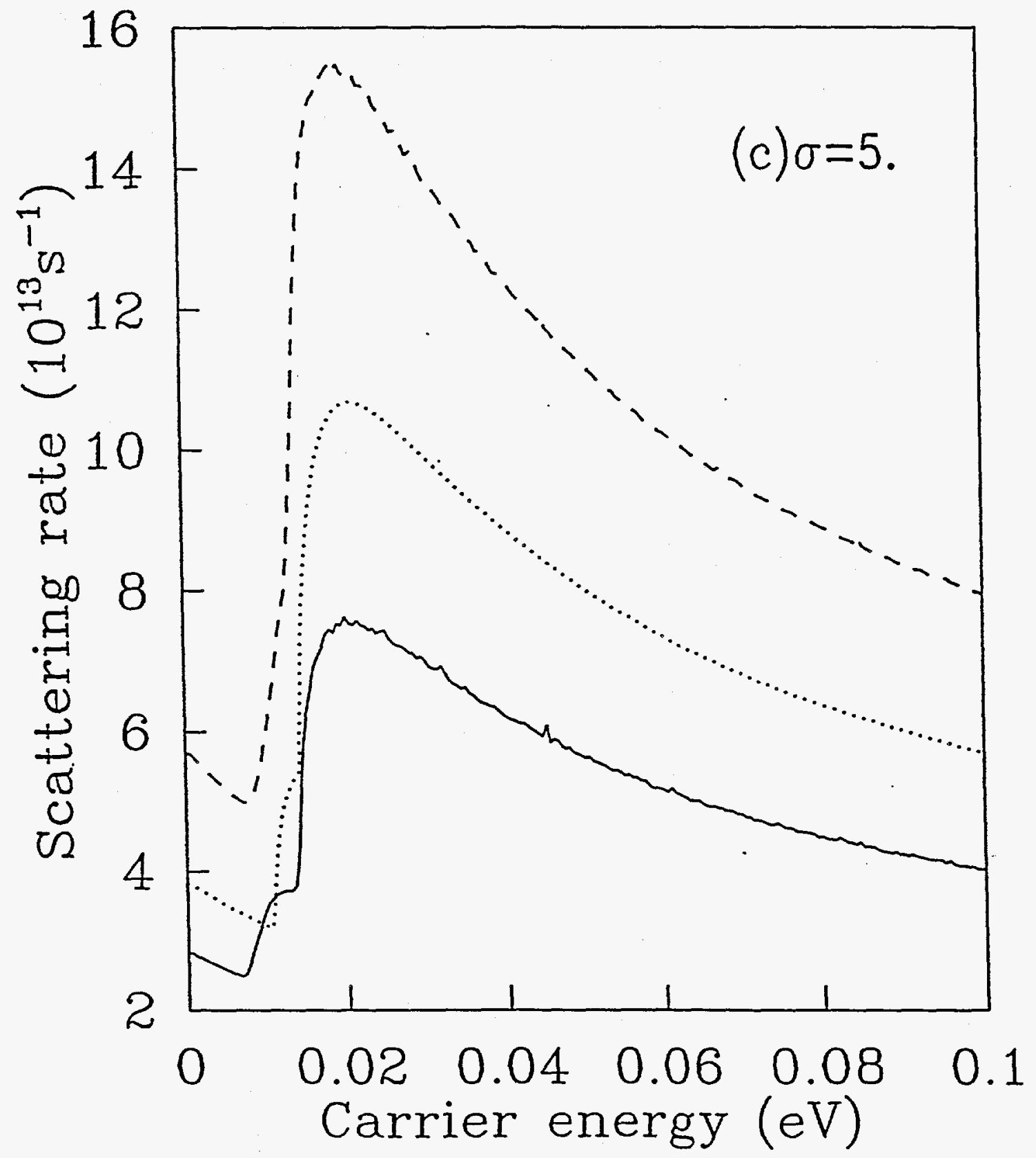

Fig. 5(c) 


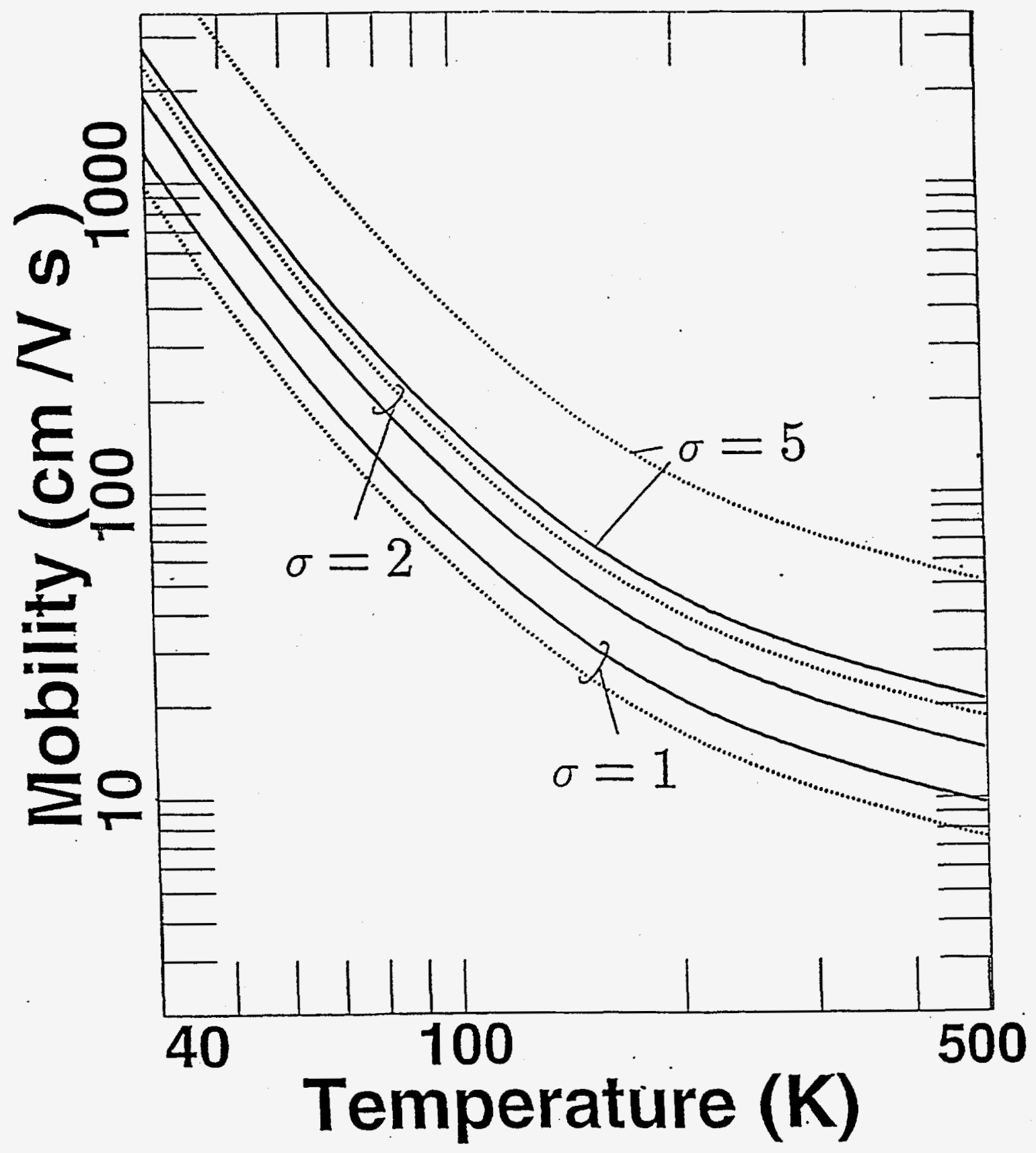


UNLIMITED RELEASE

INITIAL DISTRIBUTION:

Carnegie Mellon University

Department of Electrical and Computer Engineering

Attn: L. Salary

T. E. Schlesinger

J. Toney

Pittsburgh, PA 15213

Fisk University

Department of Physics

Attn: A. Burger

K. T. Chen

Nashville, TN 37208

University of California

Department of Materials Science and Engineering

Attn: M. Goorsky

Hojun Yoon

J. Van Scyoc

Los Angeles, CA 90024

University of Illinois at Urbana-Champaign

Department of Physics

Attn: Y.C. Chang

1110 West Greet Street

Urbana, IL 61801

University of Nebraska

Department of Electrical Engineering

Attn: H. Yao

Lincoln, NB 68588

Goddard Space Flight Center

Attn: Jack Trombka, MC 691

Greenbelt, MD 20771

RMH Inc.

Attn: K. Shah

44 Unt Street

Watertown, MA 02172

MS0459 J. M. Taylor

MS9007 A. E. Pontau

MS9056 J. Vitko

MS9141 M. A. Freudendahl

MS9161 D. F. Cowgill

MS9161 M. S. Schieber

MS9161 R. J. Anderson

MS9162 D. H. Morse

MS9402 A. J. Antolak

MS9402 D. L Medlin

MS9403 E. S. Soria 


\begin{tabular}{ll} 
MS9403 & J. C. F. Wang \\
MS9405 & J. C. Lund \\
MS9405 & J. M. Hruby \\
MS9405 & R. B. James (3) \\
MS9420 & L. A. West \\
& Attn: B. E. Affeldt, MS9040 \\
& V. C. Barr, MS9107 \\
& J. A. Fordham, MS9133 \\
& R. H. Stulen, MS9409 \\
& M. H. Rogers, MS9420 \\
& L. N. Tallerico, MS9430 \\
& A. J. West, MS9430 \\
MS9671 & B. Brunett. \\
MS9671 & E. Cross \\
MS9671 & H. Hermon (2) \\
MS9671 & R. W. Olsen \\
MS9001 & T. O. Hunter \\
& Attn: P. N. Smith, MS9002 \\
& \multicolumn{2}{c}{ D. L. Crawford, MS9003 } \\
& \multicolumn{2}{c}{ J. B. John, MS9004 } \\
& R. C. Waynt, MS9005 MS9007 \\
& W. J. McLean, MS9054 \\
& P. E. Brewer, MS9141 \\
& T. M. Dyer, MS9405
\end{tabular}

MS 9021 Technical Communications Department, 8815, for OSTI (10)

MS 9021 Technical Communications Department, 8815/Technical Library, MS 0899, 4414

MS 0899 Technical Library, $4414(4)$

MS 9018 Central Technical Files, 8940-2 (3) 